EXTRACTION OF WEAK TARGET FEATURES FROM RADAR

TOMOGRAPHIC IMAGERY

\author{
Dissertation \\ Submitted to \\ The School of Engineering of the \\ UNIVERSITY OF DAYTON
}

\begin{abstract}
In Partial Fulfillment of the Requirements for
The Degree of

Doctor of Philosophy in Engineering
\end{abstract}

By

Muhannad Salem S. Almutiry

UNIVERSITY OF DAYTON

Dayton, Ohio

August, 2016 


\section{EXTRACTION OF WEAK TARGET FEATURES FROM RADAR \\ TOMOGRAPHIC IMAGERY}

Name: Almutiry, Muhannad Salem S.

APPROVED BY:

Michael C. Wicks, Ph.D.

Advisory Committee

Professor

Electrical Engineering
Keigo Hirakawa, Ph.D.

Committee Member

Associate Professor

Electrical Engineering
John S. Loomis, Ph.D.

Committee Member

Professor

Electrical Engineering
Lorenzo Lo Monte, Ph.D.

Committee Member

Associate Professor

Electrical Engineering
Robert J. Wilkens, Ph.D., P.E.

Associate Dean for Research and

Innovation

Professor

School of Engineering
Eddy M. Rojas, Ph.D., M.A., P.E.

Dean, School of Engineering 
CCopyright by

Muhannad Almutiry

All Rights Reserved

2016 


\section{ABSTRACT \\ EXTRACTION OF WEAK TARGET FEATURES FROM RADAR \\ TOMOGRAPHIC IMAGERY}

Name: Almutiry, Muhannad Salem S.

University of Dayton

Advisor: Michael C. Wicks

Radio Frequency (RF) Tomography is a mathematical process of 3D image reconstruction from a measurement using a multistatic distribution of transmitters and receivers. The geometric diversity of these elements increases the information in the measurements. The process of determining the permittivity and conductivity profile in the measurement domain, and, therefore, the shape of the target, from the scattered field measurements, is an inverse problem. To solve this problem, under conventional methods such as the Born approximation, we use the principles of linear scattering to determine a linear relationship between measured returns and target shape. The Born approximation is valid if the scatterer is small and does not interact strongly with other objects. However, strong scatterers within the domain may generate sidelobes masking weaker returns. This masking, in conjunction with multipath effects, may result in loss of features and subsequent failure to identify a target.

In this research, a novel method is proposed to increase overall image quality and extend the capabilities of RF tomography by modeling the strong scatterers in the measurement domain as dipoles that behave as secondary sources (transmitters). Unlike conventional methods, the dipole model reduces the effects of the sidelobes from the strong scatterers 
and exploits the multipath of multiple targets or complex shapes. The multipath phenomena contains more information about the targets permitting illumination in the shadowed region and an increase to the radar aperture length. The electromagnetic characteristics for each modeled dipole are estimated by representing the cells in the measurement domain's image. The eigenvalue and eigenvector from each cell represent the phase and magnitude for the modeled dipole and also the spatial orientation of the target. The process of modeling large scatterers as dipoles can be iterated, addressing one strong scatterer at a time. This method effectively suppresses the sidelobes and exploits the multipath within the measurement domain. Using the Born approximation, the linear relationship between the scattered fields and the target is updated for simplicity. With iterations, the "extra" dipole will account for the multipath effects, thus removing some limitations caused by the Born approximation. This concept has been successfully demonstrated in software (FEKO@ by Altair). In addition, this work also presents an innovative conversion using a back-projection algorithm for multipath effects and modeling of an "additional" source or transmitter in the measurement domain. The result of implementing this method of modeling strong scatterers as dipoles successfully demonstrated an increase in the resolution and enhanced radar imagery. 
To my loving parents Salem and Maryam

My siblings Fatima, Fahad, Thamer, Khaled, Omar, Latifah and Ahad 


\section{ACKNOWLEDGEMENTS}

"In the name of God, the Beneficent, the Merciful."

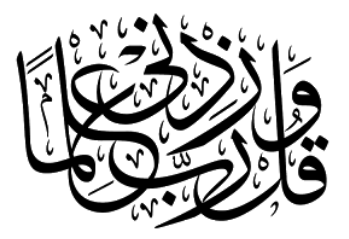

"My Lord, increase me in knowledge" [20:114]

Before all, I praise my Creator for all the favors He has bestowed upon me. I am ever grateful for all Your blessings: a beautiful family, loving siblings, supportive parents, and all other gifts I cannot even begin to list. I was able to complete my studies at University of Dayton only through the knowledge and capabilities You have given me. I thank my parents for the encouragement and unconditional love throughout my life. You have been there for me at every milestone and I can never repay this debt to you. Many students and faculty at University of Dayton provided crucial support. I especially want to thank my committee members. Dr. Wicks, my advisor for providing me with the support I needed to succeed in my endeavor. Your expertise, patience, and wisdom kept me calm and collected through this difficult process. You tempered my eagerness and kept me on track. I consider it a privilege to have worked with you. Dr. Lo Monte, thank you for your driving motivation energized me to tackle the tough problems. Dr. Hirakawa, thank you for the inspiration he provided for this research work. Dr. Loomis, thank you for you advising during my study. 


\section{PREFACE}

In this research we proposed an improved solution to two problems. The first problem is caused by the sidelobe of the dominant scatterer masking a weak scatterer. The proposed solution is to suppress the dominant scatterers by modeling its electromagnetic effects as a secondary source or "extra dependent transmitter" in the measurement domain. The suppression of the domain scatterer reveal the presence of the weak scatterer based on exploitation of multipath effects. The second problem is linearizing the mathematical forward model in the measurement domain. Improving the quantity of the prediction, including multipath scattering effects (which as neglected under the Born approximation) allows us to solve the inverse problem. The multiple bounce (multipath) scattering effects is the interaction between more than one targets in the scene. Modeling reflections from one target towards another as a transmitting dipole will add the multiple scattering effects to the scattering field, and permit us solve a linear inverse problem without sophisticated solutions of a nonlinear matrix in the forward model. Simulation results are presented to validate the concept, and a calibration procedure of unique automated semi-anechoic chamber was introduced too. 


\section{TABLE OF CONTENTS}

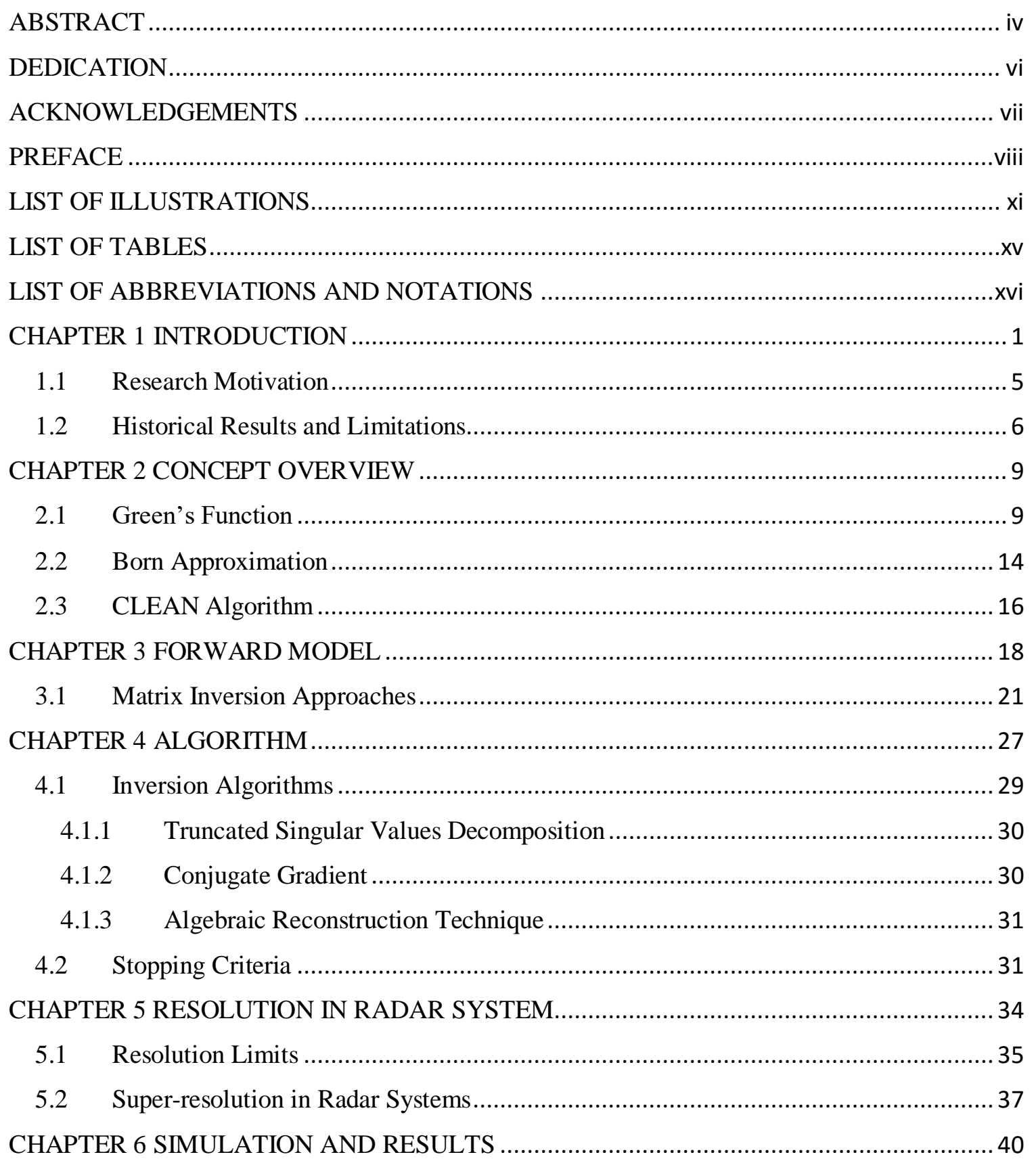


6.1 Near-field Point Spread Function of Single Target ............................................ 40

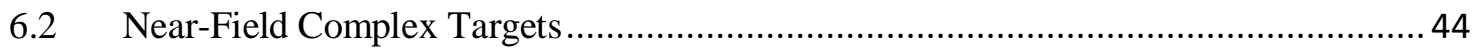

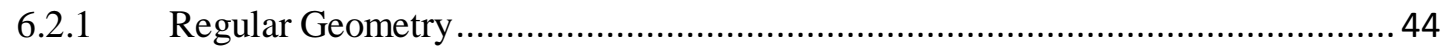

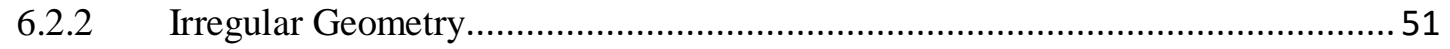

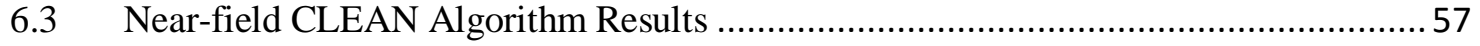

6.3.1 Regular Complex Target Shape Geometry …................................................... 57

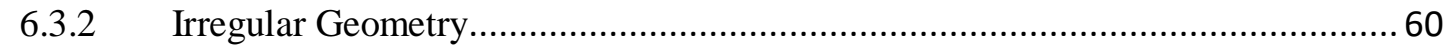

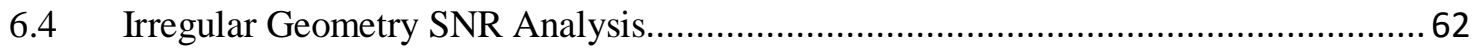

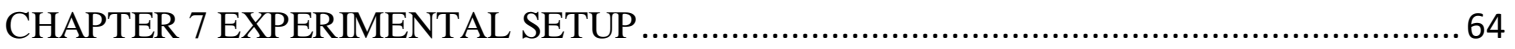

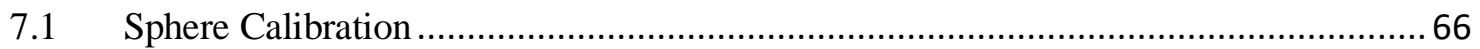

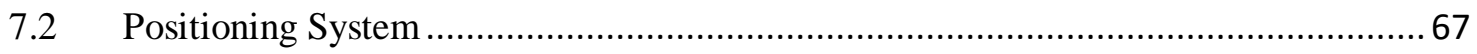

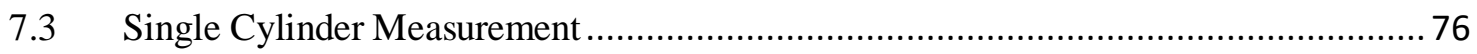

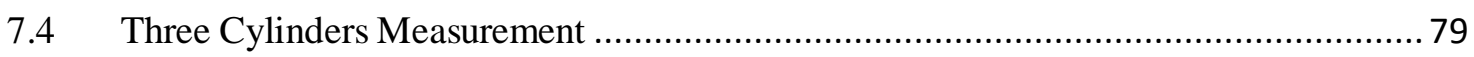

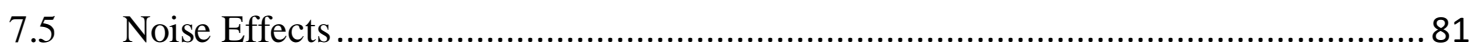

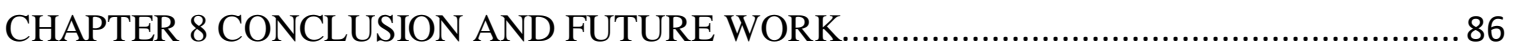

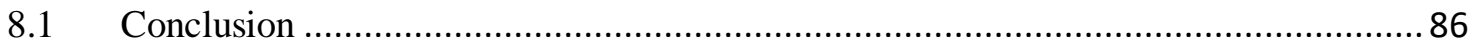

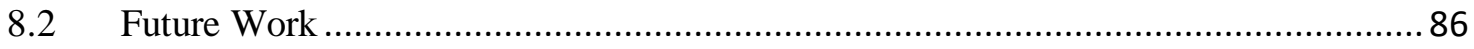

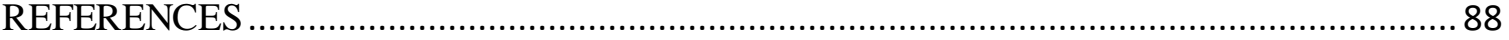




\section{LIST OF ILLUSTRATIONS}

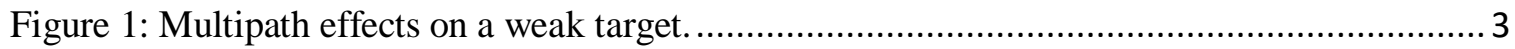

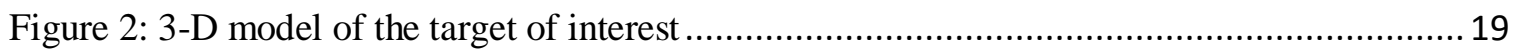

Figure 3: The formation of the measurement domain as cells............................................. 23

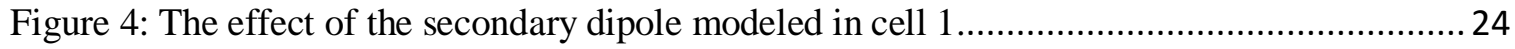

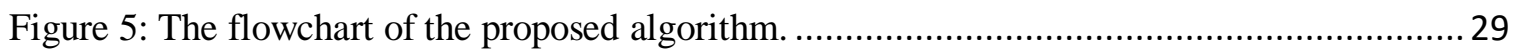

Figure 6: Experimental data of background and $5 \mathrm{~cm}$ thin wire measurement. ......................... 33

Figure 7: Closely spaced targets with the same intensity .................................................. 37

Figure 8: Dominant scatterer sidelobe masks weak scatterer............................................ 37

Figure 9: Two targets: a cylinder with radius $\lambda / 4$ located in the center and a cylinder with radius $\lambda / 50$.

Figure 10: The resulting of conventional RF tomographic image of two targets.

Figure 11: The conventional RF tomographic image is represented as cells vector of the two targets.

Figure 12: The dominant scatterer assumed to be a dipole.

Figure 13: Domain after being replaced by the dominant scatterer with a dipole.

Figure 14: Thirteen targets: twelve cylinders with radius $\lambda / 4$ located in the center as L-shape complex target and a cylinder with radius $\lambda / 50$ .46

Figure 15: Initial tomographic image reconstruction, showing only the dominant target. .46

Figure 16: Intensity 3D analysis for the contrast function before applying MRL algorithm. .47 
Figure 17: Initial tomographic image reconstruction represented as eigenvalues/eigenvectors... 47

Figure 18: The cross range of contrast function intensity of mainlobe of six cylinders at $\mathrm{x}$-axis. 48

Figure 19: The intensity of the weak scatterer at the cross range $x$-axis of the weak scatterer... .48

Figure 20: The intensity of cross-section of weak scatterer at y-axis.

Figure 21: Final tomographic image reconstruction produced by the algorithm, isolating the effect of the weak scatterer.

Figure 22: 3D intensity distribution analysis for the contrast function after applying MRL algorithm. 50

Figure 23: The sectional view of intensity distribution and sidelobe reduction at y-axis after applying MRL algorithm, weak scatterer at $0.112 \mathrm{~m}$. 50

Figure 24: The sectional view of power density and sidelobe reduction at $\mathrm{x}$-axis after applying MRL algorithm, weak scatterer at $0.1 \mathrm{~m}$. 51

Figure 25: The random distribution of 12 cylinders creating complex target. 52 Figure 26 : Initial tomographic image reconstruction of irregular complex target, showing only the dominant target before applying MRL algorithm .53 Figure 27: The intensity distribution of the contrast function, showing the reflected power over measurement domain before applying MRL algorithm. 53 Figure 28: y-axis cross range of weak scatterer, showing the sidelobe suppressing weak scatterer signal before applying MRL algorithm. 54

Figure 29: y-axis cross range of weak scatterer, showing the sidelobe suppressing weak scatterer signal before applying MRL algorithm. .54

Figure 30: Final tomographic image reconstruction for irregular complex target geometry produced by the algorithm, isolating the effect of the weak scatterer. 55 Figure 31: The power distribution of the contrast function, showing the reflected power over measurement domain after applying MRL algorithm. .55 
Figure 32: $\mathrm{x}$-axis cross range of weak scatterer, showing the sidelobe suppressing weak scatterer signal after applying MRL algorithm. 56

Figure 33: y-axis cross range of weak scatterer, showing the sidelobe suppressing weak scatterer signal after applying MRL algorithm. 56

Figure 34: The results of applying CLEAN algorithm for regular complex shape geometry. .......58 Figure 35: The power distribution of tomographic image after applying CLEAN algorithm for regular complex target shape geometry. 58

Figure 36: The sectional view of intensity of weak scatterer and sidelobe at $\mathrm{x}$-axis after applying CLEAN algorithm for regular complex target shape geometry. .59

Figure 37: The sectional view of intensity of weak scatterer and sidelobe at y-axis after applying CLEAN algorithm for regular complex target shape geometry. .59

Figure 38: The results of applying CLEAN algorithm for irregular complex shape geometry ..... 60 Figure 39: The power distribution of tomographic image after applying CLEAN algorithm for irregular complex target shape geometry. .61

Figure 40: The sectional view of power density and sidelobe at $\mathrm{x}$-axis after applying CLEAN algorithm for irregular complex target shape geometry.

Figure 41: The sectional view of power density and sidelobe at y-axis after applying CLEAN algorithm for irregular complex target shape geometry. 62

Figure 42: The anechoic chamber dimensions .66

Figure 43: Two antenna looking at the center of corner reflector in calibration process. .70

Figure 44: Four robotic arms controlled by DX100 in Mumma lab. 71

Figure 45: The results of scanning the targets in different locations 72

Figure 46: The big plate in the experiment set at three different locations.

Figure 47: Medium plate in the experiment set. .73

Figure 48: Small plate in the experiment set. .73 
Figure 49: Simulating a sphere using FEKO tools.

Figure 50: The scattering electric field of sphere versus frequency. ..................................... 74

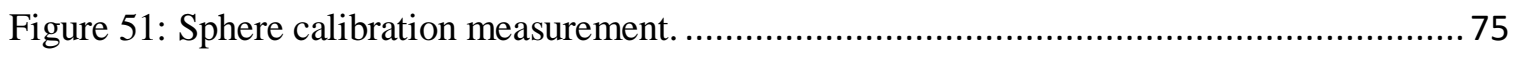

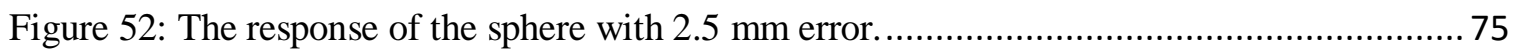

Figure 53: Measuring the scattering field for 8 inches cylinder.......................................... 77

Figure 54: The scattering field of S-parameter S12 before calibration .................................. 78

Figure 55: The scattering field of the S-parameter S12 after calibration............................... 79

Figure 56: Three cylinders placed in diagonal form for measurement. ................................... 80

Figure 57: S15 the scattered field for the first antenna and fifth antenna ............................... 81

Figure 58: S85 the scattered field for the eighth antenna and fifth antenna ............................ 81

Figure 59: Air conditioning unit installed at the anechoic chamber. ...................................... 83

Figure 60: The probability density function of the white Gaussian noise at Mumma lab............. 84

Figure 61: White Gaussian noise at Mumma lab in frequency domain. ................................. 85 


\section{LIST OF TABLES}

Table1: SNR analysis for irregular geometry. 


\section{LIST OF ABBREVIATIONS AND NOTATIONS}

\begin{tabular}{ll} 
3D & Three Dimensions \\
$\hat{a}_{r}$ & Point Sources Unit Vector Orientation \\
ART & Algebraic Reconstruction Technique \\
$D$ & Measurement Domain \\
$d$ & Eigenfunction \\
$\boldsymbol{E}(\boldsymbol{r})$ & Electric Field at location $\boldsymbol{r}$ \\
$E^{I}$ & Incident Electric Field \\
$E^{s}$ & Scattered Electric Field \\
$E^{t}$ & Total Electric Field \\
$\mathbf{e}$ & Scattering Field Vector \\
$\varepsilon_{D}$ & dielectric permittivity \\
$\mathrm{CG}$ & Conjugate Gradient \\
$G\left(r_{n}^{t}, r_{p}\right)$ & Green's Function \\
GHz & Giga Hertz \\
GPR & Ground Penetrating Radar \\
$\boldsymbol{H}(\boldsymbol{r})$ & Magnetic Field at location $\boldsymbol{r}$ \\
$\mathbf{I}$ & Identity Matrix \\
$\mathbf{L}$ & Inverse Synthetic Aperture Radar \\
& Wavenumber (Propagation Number) \\
\hline & Matrix Operator of the Mathematical Model \\
\hline &
\end{tabular}




\begin{tabular}{|c|c|}
\hline$\lambda$ & Wavelength \\
\hline$\gamma$ & Eigenvalues \\
\hline$\mu$ & Permeability \\
\hline$M$ & Number of Receivers \\
\hline $\mathrm{m}$ & meter \\
\hline$N$ & Number of Transmitters \\
\hline $\mathrm{P}$ & Number of Pixels \\
\hline PATRIOT & Phased array Tracking Radar to Intercept on Target \\
\hline PSF & Point Spread Function \\
\hline Q & Electrical Parameter of Dipole \\
\hline$r_{n}^{t}$ & Transmitting Source Location \\
\hline$r_{p}$ & Pixel Location \\
\hline$r_{m}^{r}$ & Receiving Source Location \\
\hline RF & Radio Frequency \\
\hline RFTI & Radio Frequency Tomographic Image \\
\hline$S_{i j}$ & S-parameter \\
\hline SAR & Synthetic Aperture Radar \\
\hline SNR & Signal to Noise Ratio \\
\hline$\Gamma$ & Convolution Operator \\
\hline$\Psi$ & Multiplication Operator \\
\hline$\sigma_{D}$ & dielectric conductivity \\
\hline$\tau_{\delta}\left(\mathbf{r}^{\prime}\right)$ & Contrast Function \\
\hline TR-SAR & Time Reversal Synthetic Aperture Radar \\
\hline$u$ & Eigenvector \\
\hline VNA & Vector Network Analyzer \\
\hline$\omega$ & Angular Frequency \\
\hline WWII & World War II \\
\hline$\xi(\mathbf{f})$ & Contrast Function in Frequency Domain \\
\hline
\end{tabular}




\section{CHAPTER 1}

\section{INTRODUCTION}

Exceeding the limits of human or optical aided vision by exploiting non-visual frequencies of the electromagnetic spectrum has attracted the attention of scientist for decades. Huge developments in mathematics, physics, and computing have allowed scientists to exploit radio frequency waves, infrared emission, X-ray, ultrasounds, vibrations, and other phenomena to accomplish remote sensing of objects of interest within specified environments. Even hidden or inaccessible objects such as severe weather or underground targets can now be sensed and imaged.

In general, the process of producing imagery from remote sensing technology can be described in three steps.

First, we determine the physical properties of the object to be imaged and the sensing medium [1]. For example, for tunnel detection [2], [3], man-made, synthetic material rather than stone and sand may be the desired target to be detected and imaged. For medical imaging of a tumor, the unique and different tissue densities are the targets of interest. The point is that the medium carrying the signal is very important for successfully measuring the physical dimensions of a target. However, electromagnetic waves do not require a medium to propagate. When traversing from one medium to another, the speed of transmission may be affected, also resulting in a difraction angle. Furthermore, the desired 
resolution and precision needed for accurate identification will determine which techniques are used. The acoustic wavelength of ultrasound systems for medical imaging is much shorter than electromagnetics sensors used for geology and other applixcations [4]-[6]. The short wavelength affects the image resolution. Furthermore, X-ray tomography exploits nondiffracting phenomena that improves resolution.

Second, we select a forward model that relates the data properties to the desired physical item to be reconstructed. For electromagnetic waves, measured permittivity and conductivity of the target are related to the physical characteristics. Therefore, the forward model will contain the permittivity and conductivity. Several models may be applicable. Ultimately, the selected forward model depends on the application such as X-rays or acoustics in a medical applications, sensing below underground with radar, etc.

Third, we reconstruct the image of the target by processing the data using the relationship between the measured data and physical dimensions of the object as described in the forward model. This step is called "inversion." It converts the measured data to a binary pixel format using the forward model. To handle nonlinearities between the forward model and the measured data, an approximation like Born and Rytov models are used to linearize the inversion. The approximation enables and facilitates the inversion, but may also reduce the amount of information gained from the inversion. Also, the forward model may become mathematically unsuitable due to ill-coditioned matricies, resulting in an erroneous reconstruction.

This research is focused on radar imaging applications. The general concept of radar imaging, such as multistatic synthetic aperture radar/inverse synthetic aperture radar (SAR/ISAR, ) can be viewed as radio frequency (RF) tomography. The theoretical analysis 
of multistatic radar imaging techniques shares the same framework. The common feature of multistatic radar imaging techniques is a wide distribution of transmitters and receivers. This provides more information about the shape and edges of the target. The formation of RF tomography images can be made within far-field or near-field regions. This imaging process utilizes detailed electromagnetic models of the measurement domain to estimate target echoes. The entire measurement domain is represented by a $3 \mathrm{D}$ "contrast" function, which has a nontrivial value at the boundaries between the target and the surrounding free space. Radar tomography has been proven to work in many different applications, from ground penetrating radar (GPR) to building penetration [3], [7], [8], to ISAR and even in passive modalities[9]-[12].

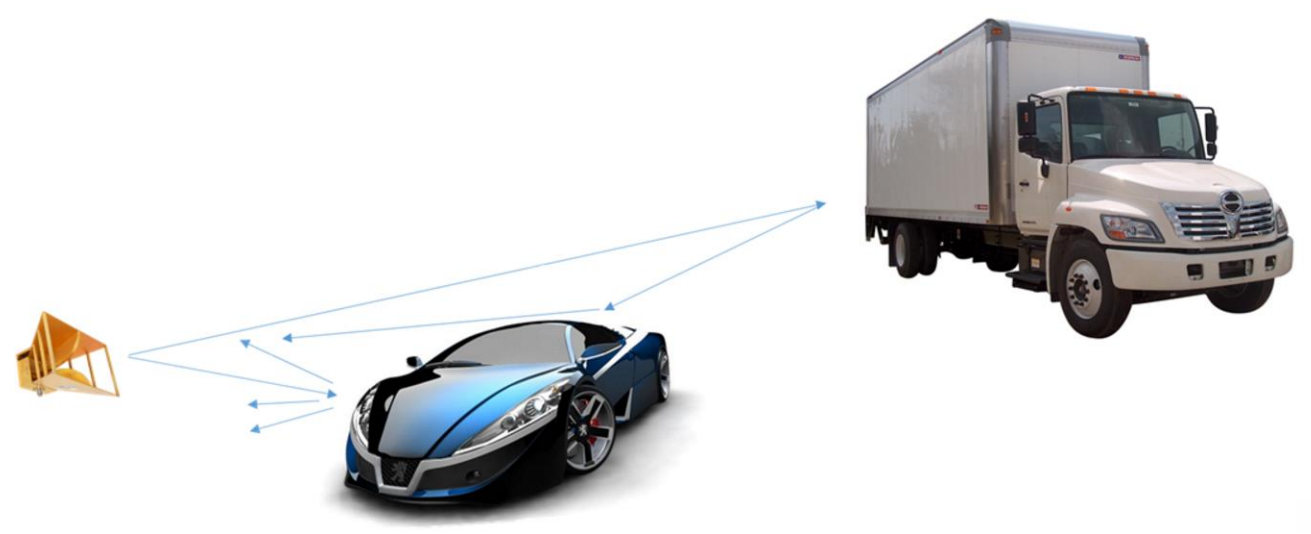

Figure 1: Multipath effects on a weak target.

RF tomography has an inherent weakness with strong scatterers that mask a nearby weak scatterer, thereby decreasing overall image quality. This is due primarily to the creation of sidelobes by the strong scatterer. There are many current techniques to ameliorate the strong scatterer echoes resulting in an enhanced image. One method is simply to remove the dominant scatterer by subtracting its point spread function signal from the received signal 
[13], [14]. The most common algorithm, using this method to suppress the dominant scatterer is the CLEAN algorithm. Moreover, the adaptive removal of modeled strong echoes has been improved by using multilook techniques to improve the effectiveness of strong echoes removal [15]. Another technique models the dominant scatterer to describe uncertainty and estimate its causes and impact on the results [16]. Modeling the dominant interfering target (or all targets) as dipoles provides more information about the shape and edges of the target as presented in [17], [18]. The estimation of the dipole polarization using the sensitivities of eigenvalues and eigenvectors has been discussed in the literature [19]. However, these algorithms are linear models that only work in far-field regions

In this research, an algorithm is proposed based on a unique electromagnetic model of the measurement domain. This approach overcomes the limitations of existing linear models. The contrast function used to represent the measurement domain consists of dipole scatterers of various strengths and polarizations. The dominant scatterers in the measurement domain are viewed as secondary transmitters that exploit the effect of the multipath caused by more than one target or a complex shape target. As a result, solving the inverse problem of the secondary transmitter is achieved by suppressing the sidelobe of the dominant scatterers. The dominant dipoles are identified and represented as eigenvalue/eigenvector pairs to provide the characteristic electromagnetic behavior. Then their scattered field is estimated, and the effect of this field on the weak scatterers is suppressed. This process utilizes a matched filter that is found by solving the inverse problem using the Born approximation. The mathematical model under the Born approximation is the key in the microwave image, the linearity of the inverse problem is proven to be the best way to reconstruct the 3D RF tomographic image [20]-[23] and 
guarantee the solution convergence. Later, the matched filter will be updated considering the dominant scatterer to be a secondary transmitter, in such a way, the dominant scatterer and weak scatterer echoes will cohere in the measurement domain as shown in Figure 1. This process is repeated in an iterative manner to suppress the effects of multiple dominant scatterers.

\subsection{Research Motivation}

Shadowing, attenuation, multipath, and dispersion all play a role in how the signals will propagate after the first interaction. Without factoring in these effects, imaging of entities within the measurement domain will be severely impacted. In addition, uncompensated refraction or diffraction through interfaces between transmission mediums can lead to localization or focusing errors, leading to image offsets and blurring [24].

Multiple or multipath scattering occurs when the targets reflects energy off other objects before being scattered back to the receiver. Multipath radar imaging (SAR, ISAR, and RF Tomography) suffers from the effects of this multiple path scattering. In conventional radar imaging, the reflections from multiple scattering centers interfere with each other, leading to a loss in resolution. Also, the energy of the multipath reflection may appear at locations where no target exists, resulting in unwanted objects, aka "ghosts" on the image [24].

Another phenomena is "masking," caused by a strong sidelobe of the dominant scatterer within the scene. The strong sidelobe dominates and essentially masks the scattering from

the lesser objects. Thus, scenes that have a single target are inherently better suited to produce accurate images, and is in concert with the direct-path reflection assumption under 
the Born approximation. The CLEAN algorithm is used to suppress strong sidelobes within a scene of interest.

Our analysis applies to near-field and far-field regions for the multipath problem. Characterizing the dominant scatterer as a dipole gives the same response of near-field scattering as well as far-field. The problem with the near-field is that the point spread function of the target is varying due viewing angle. Time reversal was one of the algorithms used in SAR imaging as (TR-SAR) for processing phase history in the presence of multiple targets. Time reversal is used in our analysis that measures the field with and without targets and then differencing techniques are applied. These techniques are known as background subtraction and are used to remove the effects of clutter and multipath. However, the clutter may not be completely suppressed by subtraction [25]. Multi-look averaging is presented in [26] to remove the effects of ghost reflections produced by multipath in radar imagery. As a results, the resolution of the averaged image may be diminished. An array of SAR images measured at different aspect angles was presented in [27] and produced using backprojection methods. The distortion of the image becomes very high at large azimuth angles [28]. Considering multipath as bistatic "jamming model” was introduced by Rosenberg and Gray [29]. Space-time adaptive processing was used in a presence of hot clutter and directpath jamming to improve the overall image quality [29]. Incoherent change detection was used in [30] to remove the multipath effects.

\subsection{Historical Results and Limitations}

The resolution of an image using diffraction tomography such as airborne SAR and GPR are theoretically limited by two factors. The first limitations, is the inversion algorithm 
itself. By solving the inverse problem and inverting the propagator matrix to find the relationship between the scattering field and the contrast function of the measurement domain, the predicted electric field response may have error. The propagator matrix, which is the set of the predicted electric field responses at each pixel location at the measurement domain, is calculated via Green's functions. Also, the inversion algorithms such as Conjugate Gradient (CG), Algebraic reconstruction technique (Kaczmarz's method) (ART), Tikhonov regularization, etc. [31] are limited by noise. Most of the inversion algorithms are iterative algorithms to compute an approximated solution of a system which converge to an estimated resolution. The second factor affecting image resolution is the approximation of the mathematical model. In radar tomographic image, the relationship between the scattering field and the scattering field of the contrast function is nonlinear. The multiple scattering effects cause the nonlinearity within the object [32]. The diffraction tomography provides accurate results when the multiple scattering effect is small, and the target in the measurement domain is weak. For the mathematical model approximation, either the Born or Rytov approximation may be used to simplify the nonlinear relationship in the inverse problem that create a contrast function in the measurement domain [33], [34]. Many algorithms exceed the limits caused by neglecting the multiple scattering effects and the weak scatterer using nonlinear algorithms such as the distorted Born iterative method, iterative extended Born method and Born iterative method [35]-[37]. The operating regions (near-field and far-field) around the electromagnetic field can change the resolution of the tomographic image. The theoretical calculation of the resolution on far-field region is limited to a wavelength between $0.3536-0.5 \lambda$. In the near-field region, the resolution of the object function is smaller in half space where it is reported to be less than $0.1768 \lambda$ [38]. 
Furthermore, any image resolution in far-field less than $0.25 \lambda$ and $0.1 \lambda$ for near-field will be considered as super-resolution phenomenon together with extracting weak scatterer features in the reconstructing object function. The next chapter explains resolution terms and the criteria for resolution definition. 


\section{CHAPTER 2}

\section{CONCEPT OVERVIEW}

Radar systems use and manage all available resources from different scientific subjects such as electromagnetic waves, signal processing, detection, and estimation. This chapter provides an overview to link all knowledge to radar imaging. This chapter starts with definitions and derivation of the Green's function. The Born approximation is reviewed since it is one of the factors limiting the image (diffraction). The last part represents CLEAN algorithm to show and compare the improvement in sidelobe reduction (in radar imaging processing in near-field region).

\subsection{Green's Function}

The electric field response of each cell in the measurement domain can be calculated by Green's functions [39], [40]. An accurate calculation of the Green's function will provide an improved image after the process. Green's functions determines the electric field response in free space of any point source at any chosen location [41]. In this research, a bistatic transmitter and receiver scenario was considered. In this scenario an isotropic source located at $r_{n}^{t}=(x, y, z)$ radiates an electromagnetic vector field in all directions in

free space. This electromagnetic field is defined as the incident field, denoted by $E^{I}$, with respect to the target. The region of interest for which an image is to be constructed is 
discretized into $P$ pixels. For a target located at $r_{p}$, electric field scatters similarly to how it is transmitted from its source. The scattered field $E^{s}$, is recorded by a receiver at $r_{m}^{r}$. Assuming that targets do not have any depolarizing effects on the incident field, then $E^{I}$ and $E^{s}$ will have the same polarization. Also, individual targets are assumed to be isotropic. These two common assumptions make it possible to simplify the vector wave equation into a scalar equation without changing its meaning.

The scattered scalar field $E^{s}$ and incident field $E^{I}$ in E together satisfy the decomposition of the total field $E^{t}$ as follows:

$$
E^{t}=E^{I}+E^{s}
$$

Regarding the transmitter-target distance, the incident field can be defined as

$$
E^{I}\left(r_{n}^{t}, r_{p}\right)=\hat{a}_{r} \cdot G\left(r_{n}^{t} \cdot r_{p}\right)
$$

$\hat{a}_{r}$ is the orientation of the dipole as a unit vector and $G\left(r_{n}^{t}, r_{p}\right)$ represents the Green's function. Where Green's function for three dimensions are calculated as [32]

$$
G\left(r_{x}, r_{y}\right)=\left(\begin{array}{lll}
G_{x x} & G_{x y} & G_{x z} \\
G_{y x} & G_{y y} & G_{y z} \\
G_{z x} & G_{z y} & G_{z z}
\end{array}\right)=\left(\mathrm{I}+\frac{\nabla \nabla}{k^{2}}\right) \frac{e^{-j k \mid r_{n}^{t}-r_{p} \|}}{4 \pi\left\|r_{n}^{t}-r_{p}\right\|},
$$

where the differential part can be written as 


$$
\left(\mathrm{I}+\frac{\nabla \nabla}{k^{2}}\right)=\left(\begin{array}{ccc}
1+\frac{\partial^{2}}{k^{2} \partial x \partial x} & \frac{\partial^{2}}{k^{2} \partial x \partial y} & \frac{\partial^{2}}{k^{2} \partial x \partial x} \\
\frac{\partial^{2}}{k^{2} \partial y \partial x} & \frac{\partial^{2}}{k^{2} \partial y \partial y} & \frac{\partial^{2}}{k^{2} \partial y \partial x} \\
\frac{\partial^{2}}{k^{2} \partial z \partial x} & \frac{\partial^{2}}{k^{2} \partial z \partial y} & 1+\frac{\partial^{2}}{k^{2} \partial z \partial z}
\end{array}\right)
$$

Let $r=\mathrm{r}_{n}^{t}-\mathrm{r}_{p}$, Then

$$
\begin{gathered}
G_{x x}=\frac{\mathrm{e}^{-\mathrm{ikr}}}{4 \pi k r}\left(\frac{3 x^{2}}{r^{5}}+\frac{3 i x^{2} k}{r^{4}}-\frac{x^{2} k^{2}+1}{r^{3}}-\frac{i k}{r^{2}}+\frac{k^{2}}{r}\right) \\
G_{x y}=\frac{\mathrm{e}^{-\mathrm{ikr}}}{4 \pi k r}\left(\frac{3 x y}{r^{5}}+\frac{3 i k x y}{r^{4}}-\frac{k^{2} x y}{r^{3}}\right) \\
G_{x z}=\frac{\mathrm{e}^{-\mathrm{ikr}}}{4 \pi k r}\left(\frac{3 x z}{r^{5}}+\frac{3 i k x z}{r^{4}}-\frac{k^{2} x z}{r^{3}}\right) \\
G_{y x}=\frac{\mathrm{e}^{-\mathrm{ikr}}}{4 \pi k r}\left(\frac{3 x y}{r^{5}}+\frac{3 i k x y}{r^{4}}-\frac{k^{2} x y}{r^{3}}\right) \\
G_{y y}=\frac{\mathrm{e}^{-\mathrm{ikr}}}{4 \pi k r}\left(\frac{3 y^{2}}{r^{5}}+\frac{3 i y^{2} k}{r^{4}}-\frac{y^{2} k^{2}+1}{r^{3}}-\frac{i k}{r^{2}}+\frac{k^{2}}{r}\right) \\
G_{z y}=\frac{\mathrm{e}^{-\mathrm{ikr}}}{4 \pi k r}\left(\frac{3 y z}{r^{5}}+\frac{3 i k y z}{r^{4}}-\frac{k^{2} y z}{r^{3}}\right) \\
G_{z z}=\frac{\mathrm{e}^{-\mathrm{ikr}}}{4 \pi k r}\left(\frac{3 x z}{r^{5}}+\frac{3 i k x z}{r^{4}}-\frac{k^{2} x z}{r^{3}}\right) \\
\left.\frac{r^{-i k r}}{r^{5}}+\frac{3 i k y z}{r^{4}}-\frac{k^{2} y z}{r^{3}}\right)
\end{gathered}
$$




$$
G_{z z}=\frac{\mathrm{e}^{-i \mathrm{kr}}}{4 \pi k r}\left(\frac{3 y^{2}}{r^{5}}+\frac{3 i y^{2} k}{r^{4}}-\frac{y^{2} k^{2}+1}{r^{3}}-\frac{i k}{r^{2}}+\frac{k^{2}}{r}\right),
$$

The electric field with respect to the Green's function at any point $r$ is

$$
\boldsymbol{E}(\boldsymbol{r})=j \omega \mu G\left(r, r^{\prime}\right) a\left(r^{\prime}\right)
$$

On the polarization of the point source, the electric field at any point $r$ will be given by below in different polarization

$$
\left[\begin{array}{c}
E_{x} \\
E_{y} \\
E_{z}
\end{array}\right]=j \omega \mu\left[\begin{array}{lll}
G_{x x} & G_{x y} & G_{x z} \\
G_{y x} & G_{y y} & G_{y z} \\
G_{z x} & G_{z y} & G_{z z}
\end{array}\right]\left[\begin{array}{l}
a_{x} \\
a_{y} \\
a_{z}
\end{array}\right],
$$

Likewise for the magnetic field,

$$
\begin{gathered}
\boldsymbol{H}(\boldsymbol{r})=\nabla x G\left(r, r^{\prime}\right) \\
{\left[\begin{array}{c}
H_{x} \\
H_{y} \\
H_{z}
\end{array}\right]=\frac{\mathrm{e}^{-\mathrm{ikr}}}{4 \pi k r}\left(\frac{1+i k r}{4 \pi r^{3}}\right)\left[\begin{array}{ccc}
0 & z-z_{1} & y_{1}-y \\
z_{1}-z & 0 & x-x_{1} \\
y-y_{1} & x_{1}-x & 0
\end{array}\right],}
\end{gathered}
$$

The electric field model of the target was defined by a single frequency to express the measurement domain $D$ and has been previously well examined [42], [43]. The 3D geometric view of the model is shown in Figure 2. The investigation domain of the target is assumed to be a homogeneous medium with estimated dielectric permittivity $\varepsilon_{D}$, conductivity $\sigma_{D}$, and permeability $\mu_{0}$. The transmitters and receivers are located in free space. The measurement domain $D$ has an unknown dielectric permittivity $\varepsilon_{r}\left(r^{\prime}\right)$ and the 
conductivity $\sigma\left(r^{\prime}\right)$ profiles, where $r^{\prime}$ is a separate position vector. The unknown dielectric permittivity contrast exhibits the inverse problem as

$$
\tau_{\delta}\left(\mathbf{r}^{\prime}\right)=\varepsilon_{r}\left(\mathbf{r}^{\prime}\right)-\varepsilon_{D}+j \frac{\sigma\left(\mathbf{r}^{\prime}\right)-\sigma_{D}}{2 \pi f \varepsilon_{0}}
$$

Where the spatial frequency of the wave (wavenumber) is the value of interest, expressed in the measurement domain $D$ can be defined as

$$
\begin{gathered}
k^{2}\left(\mathbf{r}^{\prime}\right)=\omega^{2} \mu_{0} \varepsilon_{0} \varepsilon_{r}\left(\mathbf{r}^{\prime}\right)+j \omega \mu_{0} \sigma\left(\mathbf{r}^{\prime}\right) \\
=k_{D}^{2}+k_{0}^{2} \varepsilon_{\delta}\left(\mathbf{r}^{\prime}\right) \\
k_{D}=\omega \sqrt{\mu_{0} \varepsilon_{0} \varepsilon_{r}\left(\mathbf{r}^{\prime}\right)+j \omega \mu_{0} \sigma_{D} / \omega} \\
k_{0}=\omega \sqrt{\mu_{0} \varepsilon_{0} \varepsilon_{r}\left(\mathbf{r}^{\prime}\right)}
\end{gathered}
$$

The vector wave equation for each point in the measurement domain $D$ is given by

$$
\nabla \times \nabla \times \mathbf{E}\left(\mathbf{r}^{\prime}\right)=\left[k_{D}^{2}+k_{0}^{2} \tau_{\delta}\left(r^{\prime}\right)\right] \mathbf{E}\left(\mathbf{r}^{\prime}\right)
$$

Where the scattered field $\mathbf{E}^{\mathbf{S}}\left(\mathbf{r}^{\prime}\right)$ is expressed regarding total field $\mathbf{E}\left(\mathbf{r}^{\prime}\right)$ in measurement domain as integral form

$$
\mathbf{E}^{\mathbf{S}}\left(\boldsymbol{r}^{\prime}\right)=k_{0}^{2} \iiint_{V_{i}} \overline{\mathbf{G}}\left(\mathbf{r}, \mathbf{r}^{\prime}\right) \cdot \mathbf{E}\left(\mathbf{r}^{\prime}\right) \tau_{\delta}\left(\mathbf{r}^{\prime}\right) d \boldsymbol{r}^{\prime}
$$

and the vector point $r \in D$ and $\bar{G}\left(r, r^{\prime}\right)$ is the Green's dyadic function is given by [39] 


$$
\overline{\mathbf{G}}\left(\mathbf{r}, \mathbf{r}^{\prime}\right)=\frac{1}{4 \pi}\left[\overline{\mathbf{I}}+\frac{1}{k(\mathbf{r})^{2}} \nabla \nabla\right] \frac{e^{-j k\left|\mathbf{r}-\mathbf{r}^{\prime}\right|}}{\left|\mathbf{r}-\mathbf{r}^{\prime}\right|}
$$

The Green's dyadic function is related to the radiation produced by single transmitter and provide solution for partial differential equation boundary condition [44]

$$
\nabla \times \nabla \times \overline{\mathbf{G}}\left(\mathbf{r}, \mathbf{r}^{\prime}\right)-k(\mathbf{r})^{2} \overline{\mathbf{G}}\left(\mathbf{r}, \mathbf{r}^{\prime}\right)=\overline{\mathbf{I}} \delta\left(\mathbf{r}-\mathbf{r}^{\prime}\right)
$$

\subsection{Born Approximation}

The Born approximation is used to linearize the integral in (24). This is the simplest approximation and is valid if the scatterer has small object contrast and the associated scattered field object does not interfere with another object in the measurement domain. When the scatterer to be inspected is weak in the propagation medium, Born or Rytov approximations can be used [34], [45]. The Born approximation will minimize unknown variables in (24) to be just $\tau_{\delta}\left(\mathbf{r}^{\prime}\right)$ by linearizing the inverse scattering problem under the approximation, as result, the inverse scattering problem will be solved for $\tau_{\delta}\left(\mathbf{r}^{\prime}\right)$ where the total field $\mathbf{E}\left(\mathbf{r}^{\prime}\right)$ is expressed on order form [1] as the scattered electric field can be written only in term of the incident field

$$
\begin{aligned}
& \mathbf{E}\left(\mathbf{r}^{\prime}\right)-\mathbf{E}^{I}\left(\mathbf{r}^{\prime}\right)=\Gamma \Psi \mathbf{E}\left(\mathbf{r}^{\prime}\right) \\
& \mathbf{E}\left(\mathbf{r}^{\prime}\right)=[\mathbf{I}-\Gamma \Psi]^{-1} \mathbf{E}^{I}\left(\mathbf{r}^{\prime}\right),
\end{aligned}
$$

where I refers to identity vector, the convolution and multiplication for $\tau_{\delta}\left(\mathbf{r}^{\prime}\right)$ are represented by $\Gamma \Psi$, respectively. Then, if the norm of the series is less than one, (28) can be expanded as 


$$
\mathbf{E}\left(\mathbf{r}^{\prime}\right)=(\mathbf{I}+\Gamma \Psi+\Gamma \Psi \Gamma \Psi+\ldots) \mathbf{E}^{I}\left(\mathbf{r}^{\prime}\right)
$$

The first term in (29) is considered to substitute the total field $\mathbf{E}\left(\mathbf{r}^{\prime}\right)$ with the incident field $\mathbf{E}^{I}\left(\mathbf{r}^{\prime}\right)$ according to first-order Born approximation [3], thus

$$
\mathbf{E}^{\mathbf{S}}\left(\boldsymbol{r}^{\prime}\right) \cong k_{0}^{2} \iint_{V_{i}} \overline{\mathbf{G}}\left(\mathbf{r}, \mathbf{r}^{\prime}\right) \bullet \mathbf{E}^{I}\left(\mathbf{r}^{\prime}\right) \tau_{\delta}\left(\mathbf{r}^{\prime}\right) d \boldsymbol{r}^{\prime}
$$

The Born approximation for the inverse problem used to compute the field scattered by an unknown target.

Now, the incident field expressed in term of Green's function is

$$
\mathbf{E}^{I}\left(\mathbf{r}^{\prime}, \boldsymbol{r}_{n}^{t}\right)=\mathrm{Q} \overline{\mathbf{G}}\left(\mathbf{r}^{\prime}, \boldsymbol{r}_{n}^{t}\right) \hat{\mathrm{a}}_{\mathrm{n}}^{\mathrm{t}},
$$

where $\mathrm{Q}=j \omega \mu_{0} \Delta l^{t} \mathrm{I}^{t}$ for an electrically small dipole. Moreover, the scattered field $\mathbf{E}^{S}\left(\boldsymbol{r}_{n}^{t}, \boldsymbol{r}_{m}^{r}\right)$ for a dipole with moment direction $\hat{\mathrm{a}}_{\mathrm{m}}^{\mathrm{r}}$ located at $\boldsymbol{r}_{m}^{r}$ can be expressed as

$$
\begin{gathered}
\mathbf{E}^{S}\left(\boldsymbol{r}_{n}^{t}, \boldsymbol{r}_{m}^{r}\right)=k_{0}^{2} \iiint_{V_{i}} \hat{\mathrm{a}}_{\mathrm{m}}^{\mathrm{r}} \overline{\mathbf{G}}\left(\boldsymbol{r}_{m}^{r}, \mathbf{r}^{\prime}\right) \mathbf{E}^{I}\left(\mathbf{r}^{\prime}, \boldsymbol{r}_{n}^{t}\right) \tau_{\delta}\left(\mathbf{r}^{\prime}\right) d \boldsymbol{r}^{\prime} \\
\mathbf{E}^{S}\left(\boldsymbol{r}_{n}^{t}, \boldsymbol{r}_{m}^{r}\right)=Q k_{0}^{2} \iiint_{V_{i}}\left[\hat{\mathrm{a}}_{\mathrm{m}}^{\mathrm{r}} \overline{\mathbf{G}}\left(\boldsymbol{r}_{m}^{r}, \mathbf{r}^{\prime}\right)\right]\left[\overline{\mathbf{G}}\left(\mathbf{r}^{\prime}, \boldsymbol{r}_{n}^{t}\right) \hat{\mathrm{a}}_{\mathrm{n}}^{\mathrm{t}}\right] \tau_{\delta}\left(\mathbf{r}^{\prime}\right) d \mathbf{r}^{\prime},
\end{gathered}
$$

Both the Born approximation and the wavelength of the operating frequency limit the RF tomographic image. In the literature, many approximations were introduced to overcome these limitations. The iterative Born method has been proposed to provide a numerical solution of multiple inverses and forward problems in the time domain [46]. However, the convergence and the computational load remain issues for this numerical solution. The Distorted Born iterative method accelerated the convergence of the iterative Born method by updating the Green's function during each iteration [35]. Extended Born 
approximation that Habashy et al. proposed a more accurate algorithm in low-frequency cases [33]. Later, for more accurate results higher-order extended Born approximation was presented by Cui et al. [47]. In the proposed method of this study, the limitations of the Born approximation are removed by estimating the multipath effects and suppression of the sidelobe of the dominant scatterer.

\subsection{CLEAN Algorithm}

Removing an undesired signal from the received signal to extract weak echoes was first presented in radio astronomy in the 1970's. This process called "CLEAN" removed the effects of the brighter stars within an image [48]. In the 80's the CLEAN algorithm was expanded for use in microwave imaging by Tsao and Steinberg [49]. In microwave imaging, the CLEAN algorithm designed for coherent radiation field derived from radiating transmitter antenna. Since then, the CLEAN algorithm has been further developed for detection and estimation. The general idea of CLEAN algorithms is to estimate the point spread function (radiation pattern or image response) of the dominant scatterer, then remove it from received signal. The CLEAN algorithm follows the following steps:

Step 1: Determine and select the strongest scatterer in the measurement domain and reconstructed image.

Step 2: Estimate the parameters of the Point Spread Function (PSF) of the strongest scatterer.

Step 3: Coherently subtract the estimated PSF from the image.

Step 4: Repeat steps 1-3, as required. Check on the residual scatterers in the image if it must be extracted. If yes, steps 1-3 are repeated. Otherwise, update the final CLEAN image. 
The stop condition is implemented by considering the signal energy and comparing it with the residual scatterers signal energy to apply a ratio then threshold that ratio [15].

For this study, the CLEAN algorithm was investigated by applying two techniques for image processing. First, we remove the clutter considered as a strong sidelobe. Second, determine the scattering center of the image that leads to improving the image quality. The iterative procedures of the CLEAN algorithm can attenuate the energy of the weak scatterer itself and subsequently subtracted from the image. However, the proposed algorithm accomplishes both of these objectives [50]. 


\section{CHAPTER 3}

\section{FORWARD MODEL}

Radar tomography views a target from many positions, due to the spatial distribution of transmitters and receivers. This geometric diversity increases the information obtained from the measurement domain. Multiple $(N)$ dipole transmitters and $(M)$ dipole receivers surround a region containing the targets to be imaged. Transmitter $n$ has location $\boldsymbol{r}_{n}^{t}$ and polarization $\hat{\mathbf{a}}_{\mathbf{n}}^{\mathbf{t}}$, and receiver $m$ has location $\boldsymbol{r}_{m}^{r}$ and polarization $\hat{\mathbf{a}}_{\mathbf{m}}^{\mathbf{r}}$. Figure 2 illustrates this configuration, showing the measurement domain and a single transmitter/receiver pair. At any given time, a transmitter radiates a known waveform while the remaining transmitters are left inactive. The distributed receivers collect the echoes from the targets. The position of each receiver is known up to a fraction of the wavelength, and all the receivers share the same reference signal [1]. The information obtained from each receiver/transmitter pair is stored after mitigating noise (and clutter).

Using this geometry, a simple forward and inverse model for the time-harmonic electric field can be produced. Note that time-domain waveforms can be expressed in time-harmonic fields using stretch processing or simply the Fourier transform. 


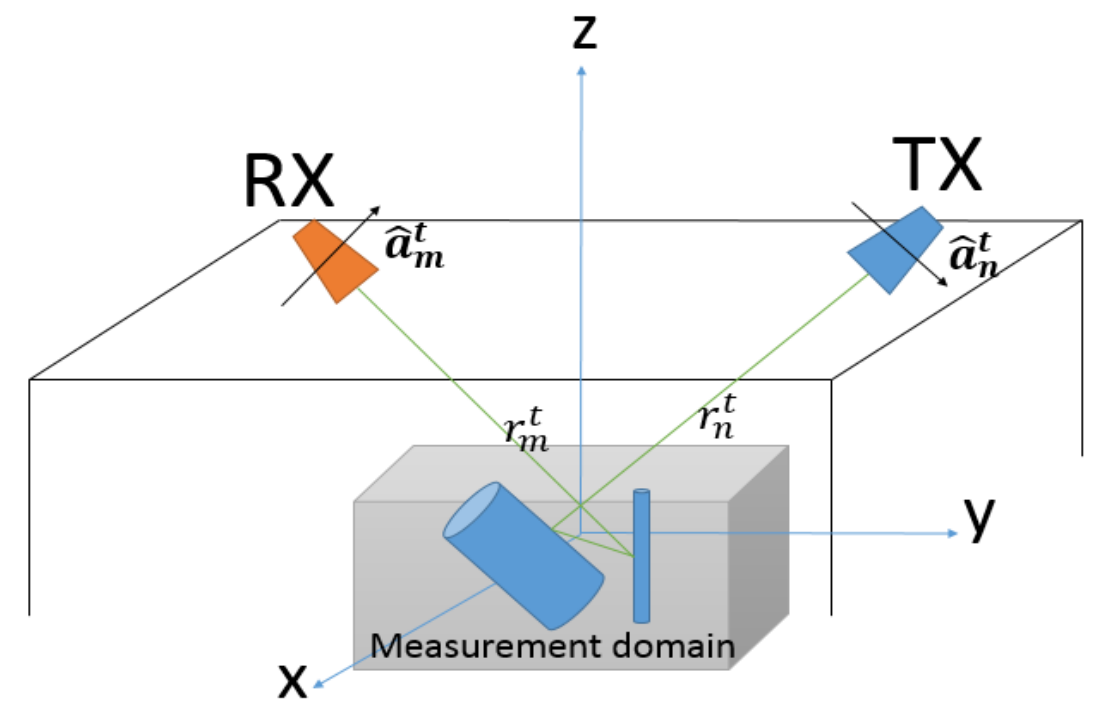

Figure 2: 3-D model of the target of interest

The measurement domain is assumed to be a free space medium with an unknown contrast function $\tau_{\delta}\left(r^{\prime}\right)$ where $r^{\prime}$ is a position vector. The scattered field $\mathbf{E}^{S}\left(\boldsymbol{r}_{n}^{t}, \boldsymbol{r}_{m}^{r}\right)$ for a transmitter $n$ and receiver $m$ can be expressed, using the Born approximation, as the integral in (33) [42]. In this equation, $\overline{\mathbf{G}}\left(\boldsymbol{r}_{m}^{r}, \mathbf{r}^{\prime}\right)$ is the Green's function, and $k$ is the wavenumber. The Born approximation is linearly related to the contrast function, and equation (33) can be represented as a matrix multiplication. In this case, the relationship can be expressed as

$$
\mathbf{E}^{S}\left(\boldsymbol{r}_{n}^{t}, \boldsymbol{r}_{m}^{r}\right)=\mathbf{L}\left(\tau_{\delta}\left(\mathbf{r}^{\prime}\right)\right)
$$

where multiplication by matrix $\mathbf{L}$ implements the forward model. The operator $\mathbf{L}$ has to be inverted to compute the unknown contrast function. Because $\mathrm{L}$ is not square and is usually ill-conditioned, this is a difficult task. However, in the literature, many approaches are 
provided for inverting the linear operator $\mathbf{L}$ [44], [51], [52]. One commonly used method is a simple inversion using the Hermitian of the linear operator $\mathbf{L}$, which is effective for dealing with ill-conditioned operators.

The contrast function $\tau$ in (34) is obtained from the multiplication of the Hermitian linear operator as an inverse problem solution. The linear operator acts as a filter on the spatial harmonics of the reconstructed contrast function. This image is a vector image where each cell could be represented as a dyadic function of three dimensions, expressed as $[x x, x y, x z, y x, y y, y z, z x, z y, z z]$. The eigenvectors $u$ and eigenvalues $\gamma$ for each cell are obtained, such that

$$
d=\gamma \boldsymbol{u},
$$

In this equation, $d$ is the cell's vector representation such each pixel has its phase and magnitude giving by the eigen-analysis. After the contrast function $\tau$ is obtained, its corresponding eigenvalues and eigenvectors are generated. Theses provide the required information to estimate the polarization and magnitude of the dominant scatterers. There are three eigenvalues for each cell. The largest eigenvalue $\gamma$ contains the most important information about the polarization and the magnitude of the dominant scatterer. After determining the $d$ scatterers field's locations at $p$ (pixel) such that $r_{p}^{d}(x, y, z)$, the scattering field response is estimated for each dominant scatterer. Since the linear operator $\mathrm{L}$ has been updated, the scattering field of the new transmitter illuminating the measurement domain is also updated as

$$
\mathbf{E}_{d}^{S}\left(\boldsymbol{r}_{n}^{t}, \boldsymbol{r}_{m}^{d}\right)=Q k_{0}^{2} \iiint_{V_{i}}\left[\hat{\mathrm{a}}_{\mathrm{m}}^{\mathrm{r}} \overline{\mathbf{G}}\left(\boldsymbol{r}_{m}^{r}, \mathbf{r}^{\prime}\right)\right]\left[\overline{\mathbf{G}}\left(\mathbf{r}^{\prime}, \boldsymbol{r}_{p}^{d}\right) \hat{\mathrm{a}}_{\mathrm{n}}^{d}\right] \tau_{\delta}\left(\mathbf{r}^{\prime}\right) d \mathbf{r}^{\prime},
$$


Now, the multipath causing the dominant scatterer is considered in the Green's function calculation. The polarization of the dominant scatterer not usually as the transmitters and receivers, so it can take any orientation in the measurement domain. The dominant scatterer electric field $\mathbf{E}_{d}^{S}$ can be either calculated theoretically or simulated. The relationship between the contrast function and the scattering field remains linear in equation (36), but the accuracy of the mathematical model under the Born approximation has been improved since the multiple scattering effects is considered in the linear operator $\mathbf{L}$.

\subsection{Matrix Inversion Approaches}

The matrix form of the contrast function of the measurement domain is described as the inverse problem. A linear relationship is applied to obtain the contrast function from the electric field response of the transmitters and receivers at each location in the measurement domain. The Radio Frequency Tomographic Image (RFTI) is represented as cells where each cell represents a contrast value at a specific location. The measurement domain has $\mathrm{N}$ transmitters and $\mathrm{M}$ receivers. The contrast function, representing a tomographic image, divides the measurement domain into individual pixel cells ( $p t h$ pixel). The scattered field is represented as a column vector of [MN X 1]. By applying the matrix from of (33)

$$
\mathbf{E}^{S}\left(\boldsymbol{r}_{\boldsymbol{n}}^{t}, \boldsymbol{r}_{\boldsymbol{m}}^{\boldsymbol{r}}\right) \cong Q \sum_{p=1}^{P}\left[\hat{\mathrm{a}}_{\mathrm{m}}^{\mathrm{r}} \overline{\mathbf{G}}\left(\boldsymbol{r}_{\boldsymbol{m}}^{r}, \mathbf{r}_{p}\right)\right]\left[\overline{\mathbf{G}}\left(\mathbf{r}_{p}, \boldsymbol{r}_{\boldsymbol{n}}^{t}\right) \hat{\mathrm{a}}_{\mathrm{n}}^{\mathrm{t}}\right] \tau_{\delta}\left(\mathbf{r}_{p}\right)
$$

Where $\mathbf{r}_{p}$ is the location of the pth pixel (cell) of $P$ number of pixels (cells) in the measurement domain. The equation (37) is the forward model of the linear relationship between the contrast function of the measurement domain and the measured scattering field 
under Born approximation. Figure 3 shows the orientation of the transmitters and the receivers can be expressed as [17]

$$
\begin{aligned}
E_{11} & =G_{11}^{T} G_{11}^{R} V_{1}+G_{12}^{T} G_{12}^{R} V_{2}+\cdots+G_{1 P}^{T} G_{1 P}^{R} V_{P} \\
E_{12} & =G_{11}^{T} G_{21}^{R} V_{1}+G_{12}^{T} G_{22}^{R} V_{2}+\cdots+G_{1 P}^{T} G_{2 P}^{R} V_{P} \\
& \vdots \\
E_{N M} & =G_{N 1}^{T} G_{M 1}^{R} V_{1}+G_{N 2}^{T} G_{M 2}^{R} V_{2}+\cdots+G_{N P}^{T} G_{M P}^{R} V_{P} .
\end{aligned}
$$

The matrix form of (38) is represented as

$$
\left[\begin{array}{c}
E_{11}^{S} \\
E_{12}^{S} \\
\vdots \\
E_{N M}^{S}
\end{array}\right]=\left[\begin{array}{cccc}
G_{11}^{T} G_{11}^{R} & G_{12}^{T} G_{12}^{R} & \cdots & G_{1 P}^{T} G_{1 P}^{R} \\
G_{11}^{T} G_{21}^{R} & G_{12}^{T} G_{22}^{R} & \cdots & G_{1 P}^{T} G_{2 P}^{R} \\
\vdots & \vdots & \ddots & \vdots \\
G_{N 1}^{T} G_{M 1}^{R} & G_{N 2}^{T} G_{M 2}^{R} & \cdots & G_{N P}^{T} G_{M P}^{R}
\end{array}\right]\left[\begin{array}{c}
V_{1} \\
V_{2} \\
\vdots \\
V_{P}
\end{array}\right]
$$

The scattering field vector is $M N \times 1$, the linear operation $\mathbf{L}$ is $M N \times P$ matrix, the contrast function vector is $P \times 1 . E_{M N}^{S}$ is the scattering electric field collected at $M N$ position. $V_{P}$ is denoting the electric field at location $P$ measured by volt as function of the target permittivity profile in permittivity background medium ( free space in the case) to create an image at final stage. $G^{T} G^{R} V$ is representing the contribution of a single transmitter and receiver to the pixel value, $G^{T}$ is calculated electric field response from the transmitter to a single point location at the measurement domain. The calculated electric field of a single receiver to a point location is denoted by $G^{R}$. The sum of the multiplication of these quantity will be equivalent to the scattering electric field received by the transmitters. 


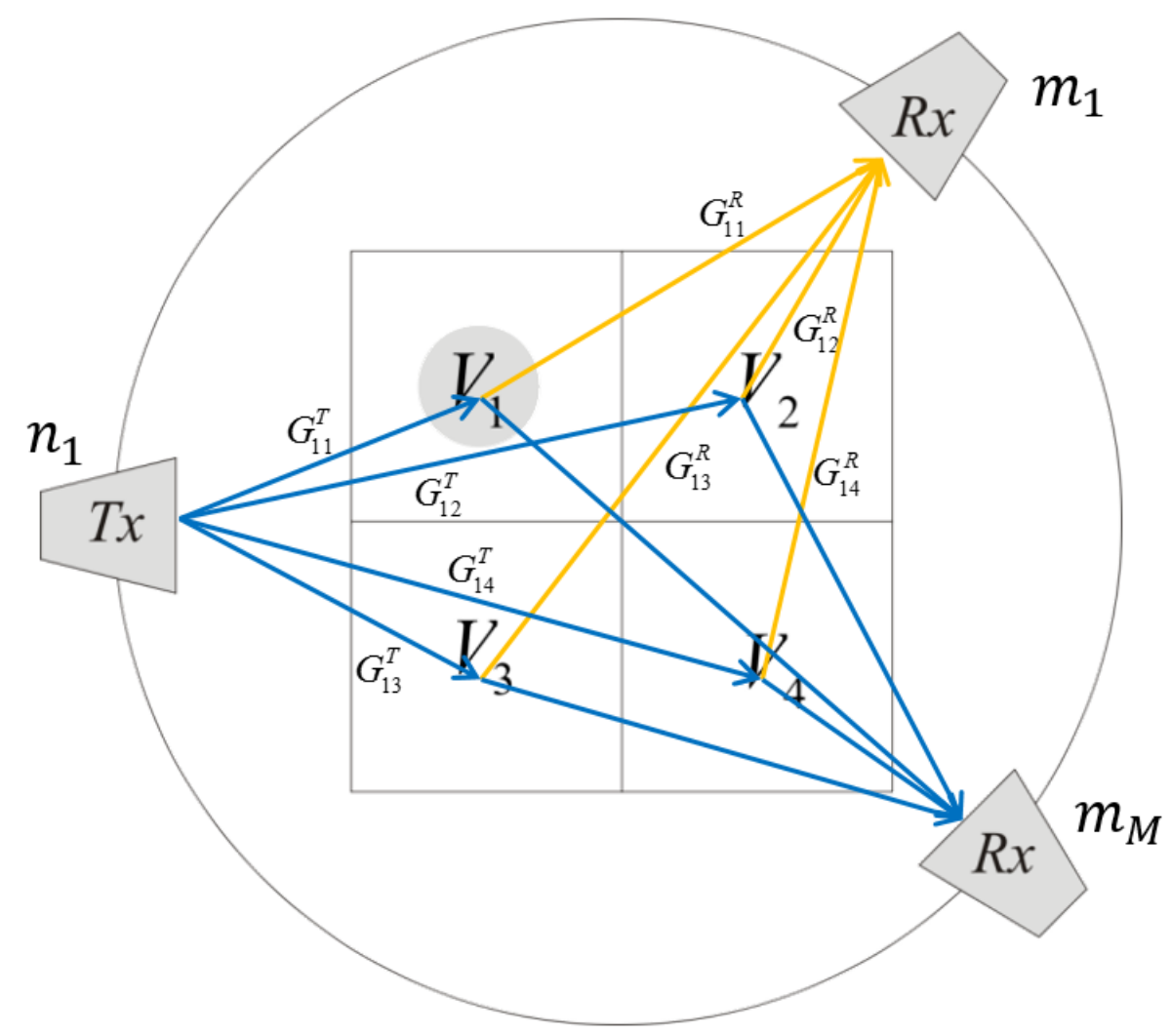

Figure 3: The formation of the measurement domain as cells. 


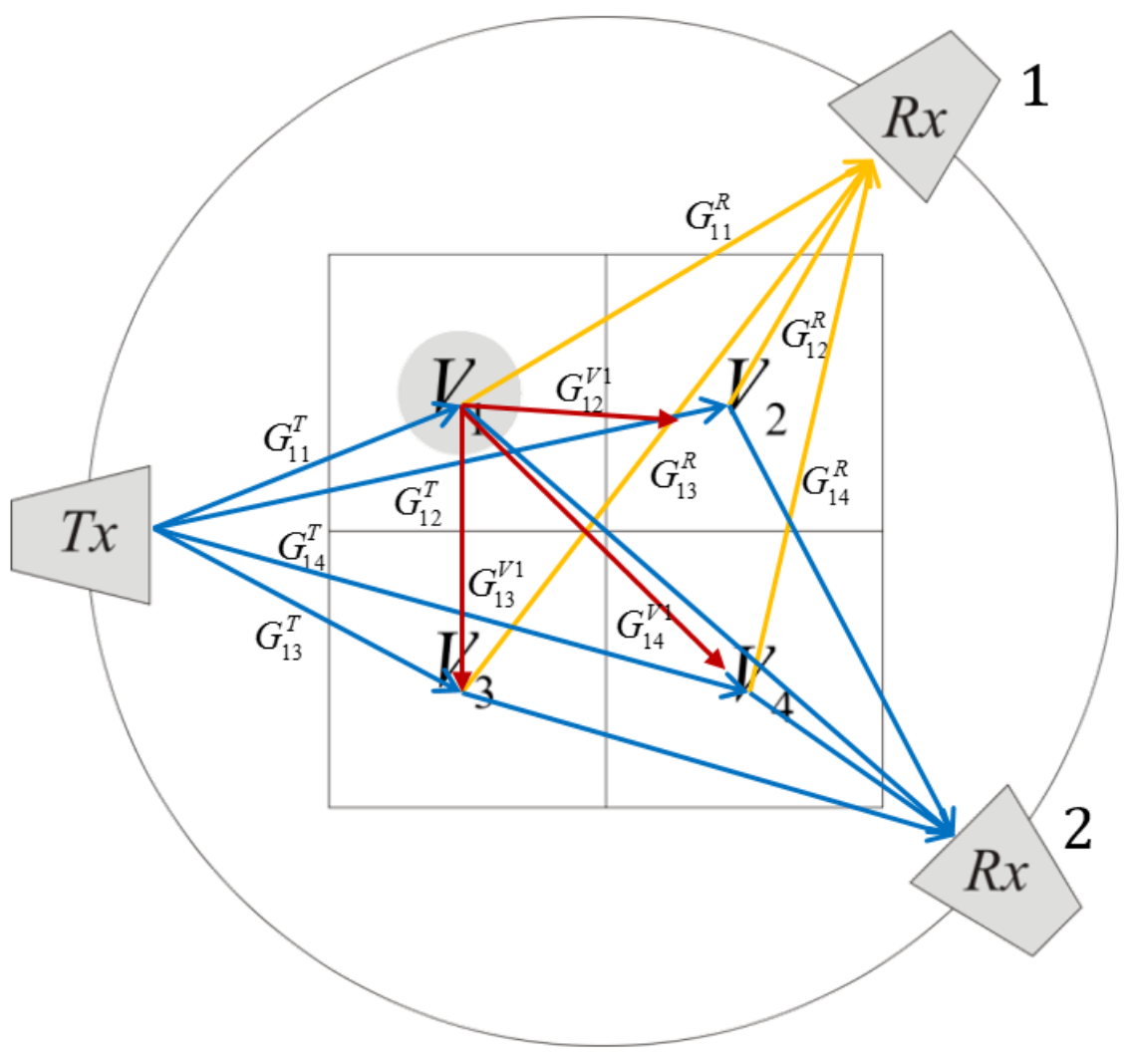

Figure 4: The effect of the secondary dipole modeled in cell 1.

Since $\mathbf{L}$ ( the multiplication of the Green's function values) is not a square matrix and illposed, a Hermitian transpose matrix can be employed to obtain the contrast vector $\mathbf{v}$ as inverse problem solution in vector notation

$$
\mathbf{v}=\mathbf{L}^{H} \mathbf{e},
$$

Other inversion techniques are presented to deal with ill-posed condition matrix.

The multiplication of $\mathbf{e}$ by $\mathbf{L}^{H}$ in this case performs the convolution of the transmitted waveform within the contrast function. As the first dominant scatterer location identified and its electromagnetics effects in the measurement domain are estimated as delayed version of the transmitted signal. 


$$
\begin{aligned}
& E_{11}^{d}=G_{11}^{d} G_{11}^{R} V_{1}+G_{12}^{d} G_{12}^{R} V_{2}+\cdots+G_{1 P}^{d} G_{1 P}^{R} V_{P} \\
& E_{12}^{d}=G_{11}^{d} G_{21}^{R} V_{1}+G_{12}^{d} G_{22}^{R} V_{2}+\cdots+G_{1 P}^{d} G_{2 P}^{R} V_{P} \\
& \vdots \\
& E_{1 M}^{d}=G_{11}^{d} G_{M 1}^{R} V_{1}+G_{12}^{d} G_{M 2}^{R} V_{2}+\cdots+G_{1 P}^{d} G_{M P}^{R} V_{P},
\end{aligned}
$$

Where $E_{1 M}^{d}$ denoted the electromagnetics modeling of first dominant scatterer. The iterative process to locate all $i$ number of dominant scatterers take a place by updating the L operator. Each step will add one dominant scatterer illuminating in the domain as new transmitter on the measurement domain. After locate all dominant scatterers in the measurement domain the $\mathbf{L}$ operator will be as

$$
\begin{aligned}
E_{11}= & G_{11}^{T} G_{11}^{R} V_{1}+G_{12}^{T} G_{12}^{R} V_{2}+\cdots+G_{1 P}^{T} G_{1 P}^{R} V_{P} \\
E_{12} & =G_{11}^{T} G_{21}^{R} V_{1}+G_{12}^{T} G_{22}^{R} V_{2}+\cdots+G_{1 P}^{T} G_{2 P}^{R} V_{P} \\
& \vdots \\
E_{N M} & =G_{N 1}^{T} G_{M 1}^{R} V_{1}+G_{N 2}^{T} G_{M 2}^{R} V_{2}+\cdots+G_{N P}^{T} G_{M P}^{R} V_{P} \\
E_{11}^{d} & =G_{11}^{d} G_{11}^{R} V_{1}+G_{i 2}^{d} G_{22}^{R} V_{2}+\cdots+G_{i P}^{d} G_{M P}^{R} V_{P} \\
& \vdots \\
E_{i M}^{d} & =G_{i 1}^{d} G_{i 1}^{R} V_{1}+G_{i 2}^{d} G_{22}^{R} V_{2}+\cdots+G_{i P}^{d} G_{M P}^{R} V_{P} .
\end{aligned}
$$

The matrix of the scattering field vector is $(N+i) M \times 1$, the relationship between the scattering filed and the contrast function of the measurement domain is kept linear by updating the operator $\mathbf{L}$ which is a matrix of $(N+i) M \times P$ shown as

$$
\left[\begin{array}{c}
E_{11}^{S} \\
E_{12}^{S} \\
\vdots \\
E_{N M}^{S} \\
E_{11}^{d} \\
\vdots \\
E_{i M}^{d}
\end{array}\right]=\left[\begin{array}{cccc}
G_{11}^{T} G_{11}^{R} & G_{12}^{T} G_{12}^{R} & \cdots & G_{1 P}^{T} G_{1 P}^{R} \\
G_{11}^{T} G_{21}^{R} & G_{12}^{T} G_{22}^{R} & \cdots & G_{1 P}^{T} G_{2 P}^{R} \\
\vdots & \vdots & \ddots & \vdots \\
G_{N 1}^{T} G_{M 1}^{R} & G_{N 2}^{T} G_{M 2}^{R} & \cdots & G_{N P}^{T} G_{M P}^{R} \\
G_{11}^{d} G_{11}^{R} & G_{12}^{d} G_{22}^{R} & \cdots & G_{1 P}^{d} G_{M P}^{R} \\
\vdots & \vdots & \ddots & \vdots \\
G_{i 1}^{d} G_{i 1}^{R} & G_{i 2}^{d} G_{22}^{R} & \cdots & G_{i P}^{d} G_{M P}^{R}
\end{array}\right]\left[\begin{array}{c}
V_{1} \\
V_{2} \\
\vdots \\
\vdots \\
\vdots \\
\vdots \\
V_{P}
\end{array}\right] .
$$


The final $\mathbf{L}$ in (43) is representing the multiple scattering effects of dominant scatterers considered in the inverse problem under Born approximation. 


\section{CHAPTER 4}

\section{ALGORITHM}

The algorithm begins by forming a radio frequency tomographic image by inverting the

linear operator $\mathbf{L}$ under Born approximation to the scattered field $E^{S}$ generating the set of eigenvalues for each cell of the resulting reconstruction by equation (35). The maximum eigenvalue that is larger than a specified threshold is used to locate the dominant scatterer in the reconstruction array. This scatterer is then modeled as a dipole. Corresponding eigenanalysis of the dominant scatterer will provide orientation to model the dipole polarization [53]. This dipole can be treated as an extra transmitter in the measurement domain. The simulated field generated by this dipole acting alone is denoted as $E_{d}^{S}$. This quantity is then processed to update the operator $\mathbf{L}$, while the linearity is kept under Born approximation. The extra modeled dipole scattered field effects is added to $E^{S}$ vector. The forward model is then updated by the addition of the dipole, treating it as a new transmitter, leading to a modified $\mathbf{L}$ operator. The signal emitted by the dipole is a scaled and delayed version of the signal from the active transmitter. This leads to interference at the receivers, determined by the wavelength and path length differences involved. This updated operator is then used in the matrix approach inversion, applying it to the modified scattered field creating a reconstruction that has the dominant scatterer removed. As the block diagram shows in 
Figure 5, this process then repeats to identify and remove the next dominant scatterer from the previous set of transmitters, including the previously modeled dipoles. The process stops when there is no value more than the preset threshold or each the noise floor noise value.

This algorithm generates a series of reconstructions, and each subsequent reconstruction shows more of the weaker scatterers. This set of reconstructions is then combined into a single reconstruction of the measurement domain contrast function, showing both the strong and weak scatterers. 


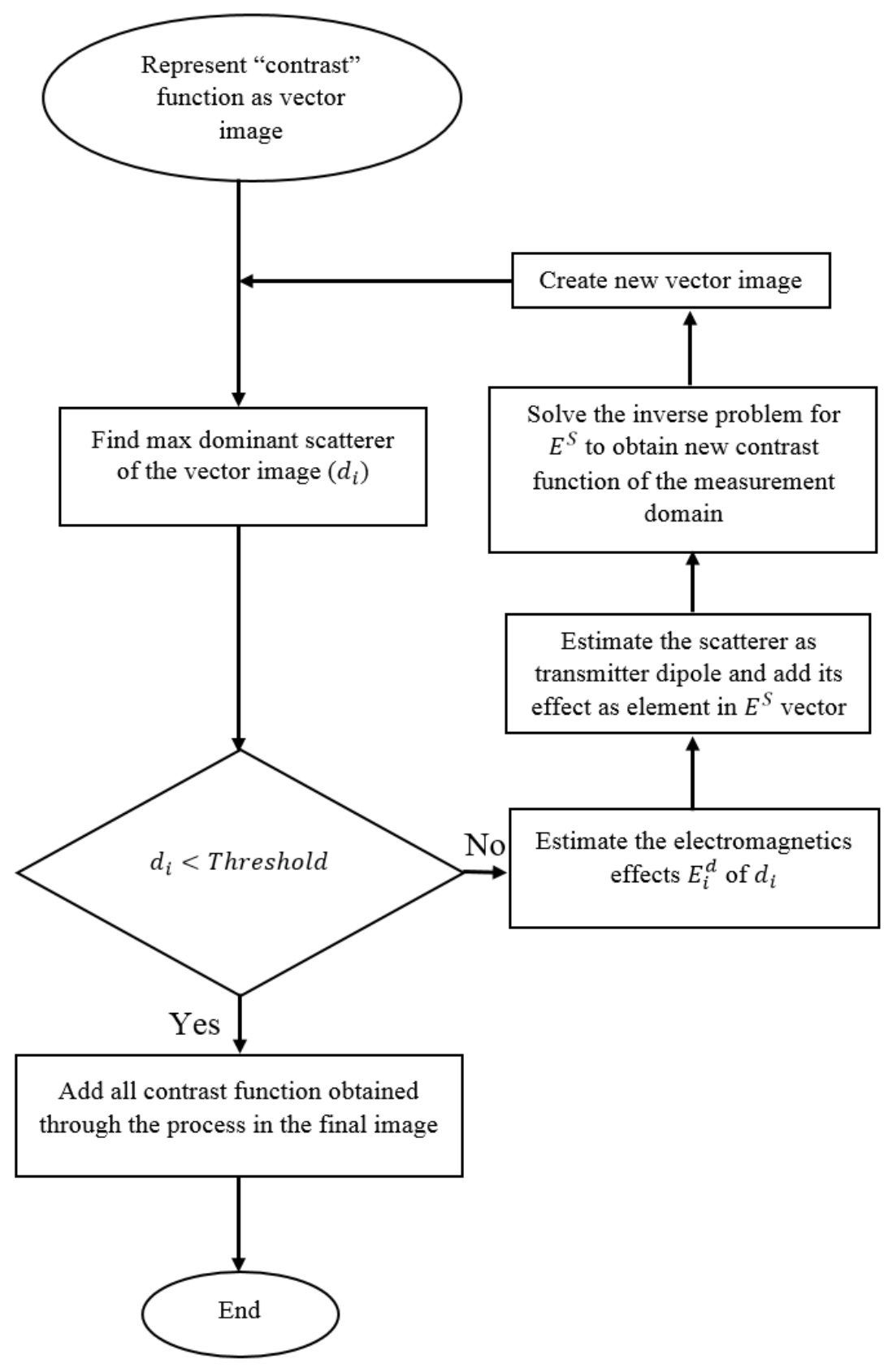

Figure 5: The flowchart of the proposed algorithm.

\subsection{Inversion Algorithms}

Radar tomographic imaging can be considered as an electromagnetic inverse problem. In general, the inverse problem is nonlinear where the nonlinearity comes from the multiple 
scattering effects. Depending on the application, the linear relationship can be found and used for simplification. In this research, Born and Rytov derived a linear Fourier transform relationship between the scattered field and object contrast function. In other applications such as computed tomography using X-Ray, the linear relationship has been found between the attenuation factor of the X-Ray and the reduction of X-Ray beam intensity after traverses matter. Even after linearizing, the relationship to find the contrast object function is still a complex problem to invert. Moreover, the $\mathrm{L}$ matrix operator is the mathematical model of the real world behavior where a good approximation of this model will lead to a good imaging system. Unfortunately, rectangular matrix L operator is not possible to be inverted directly because it is ill-conditioned. However, the inversion can be done using a pseudo-inverse matrix in form of $\mathbf{L}^{\dagger}=\mathbf{L}^{\mathbf{H}}\left(\mathbf{L} \mathbf{L}^{\mathbf{H}}\right)^{-1}$. However, a disadvantage of the pseudoinverse technique is that a small amount of noise can lead to large errors in the contrast function calculation. The preferred method to reconstruct the contrast object function while disregarding the divergence caused by small noise is the regularization techniques. The following inversion algorithms use regularization techniques described throughout the literature.

\subsubsection{Truncated Singular Values Decomposition}

This method works by decomposing the matrix $\mathrm{L}$ as $\mathbf{L}=\mathbf{U S V}^{\mathbf{H}}$, where $\mathbf{S}$ is the diagonal matrix contains the singular values $\mathbf{L}, \mathbf{U}$ and $\mathbf{V}$ are unity matrix. The pseudoinverse can be easily represented as $\mathbf{L}^{\dagger}=\mathbf{V S}^{-1} \mathbf{U}^{\mathbf{H}}$. Later, the regularized truncated pseudoinverse is calculated by $\mathbf{L}_{\mathbf{k}}^{\dagger}=\mathbf{V}_{\mathbf{k}} \mathbf{S}_{\mathbf{k}}^{-1} \mathbf{U}_{\mathbf{k}}^{\mathbf{H}}$. Where k represents the retained singular values.

\subsubsection{Conjugate Gradient}


Conjugate Gradient is an algorithm to solve a large system of linear equations based on the steepest descent method. The method is to simply represent $A x=b$, where $\mathrm{x}$ is unknown vector (the object contrast function in this research), b is a known vector (scattering field $\mathrm{E}$ ), and $\mathrm{A}$ is known (the matrix $\mathrm{L}$ operator). Now, the steepest descent method starts with an initial guess. Then an iterative method seeks to optimize the direction until the minimum gradient is reached, while the gradient of the function is computed.

\subsubsection{Algebraic Reconstruction Technique}

The Algebraic Reconstruction Technique (ART) method is very popular within biomedical imaging. ART had been used to reconstruct computed tomography of X-ray since the 1970's. The method is based on pseudo-inverse of matrix L. It is a double iterative loop procedure. First, the inner loop iteration starts from the initial guess. The second loop iterative uses the output image to start an initial guess again.

\subsection{Stopping Criteria}

The algorithm aimed be iterative process to model and suppress dominant scatterers in the measurement domain. The stop condition to determine whether there is a dominant scatterer or not is implemented by three methods. First is by thresholding the pixel values to determine the target. The maximum pixel value can be considered as the reference value to set its partial value as threshold. The second method is by considering the contrast function power of the measurement domain. The sidelobe of each iteration will be suppressed resulting degrading the total energy of the contrast function. By setting a partial energy value of first obtained contrast function energy to be the stopping criteria such these following steps. 
Step 1: Calculate the initial contrast function energy.

Step 2: Calculate iteratively the contrast function energy after applying MRL algorithm.

Step 3: Compare the ratio of step 1 and step 2 with preset threshold as partial energy of step 1 .

Step 5: if the ratio is less than the preset threshold.

The last method by considers the contrast function statistics. The following steps can be applied.

Step 1: Determine the background as Gaussian distribution of target absent (e.g. Figure 6)

Step 2: Perform statistical analysis for each pixel value to determine if that value represent target present or just Gaussian distribution noise.

Step 3: if target present, MRL is performed to suppress and exploit the sidelobe.

Step 4: If all pixels values represent a Gaussian noise distribution, then no scatterer is detected. The algorithm stop. 


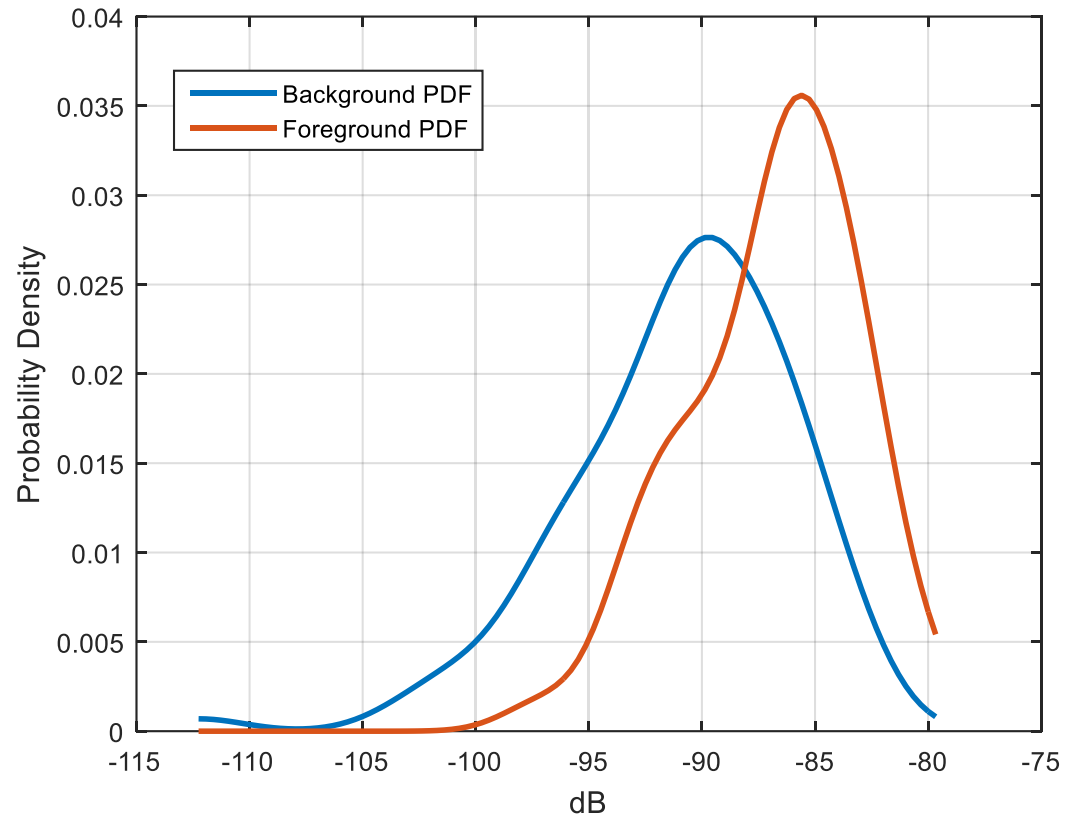

Figure 6: Experimental data of background and $5 \mathrm{~cm}$ thin wire measurement. 


\section{CHAPTER 5}

\section{RESOLUTION IN RADAR SYSTEM}

Although resolution of distinguish contiguous targets was not the initial objective of early radar functions, but it was widely pursued soon after radar was invented. The first task for radar systems was simply to detect the presence of a target. Determining target velocity and direction were not viable functions at that time. After WWII, radar systems capabilities advanced rapidly and become more sophisticated in term of functions and utilities [54]. Soon radar systems gained an ability to detect and locate targets using range resolution to detect small adjacent targets [55], [56]. Range resolution refers to the width of transmitted that can distinguish two or more targets at different range. In the advanced radar system, imaging become one of the main functions that help to distinguish the target shape and features such anti-ballistic missile system (PATRIOT) which is imaging the target to track and control the firing function of PATRIOT [57]-[59]. The angular resolution in radar system was considered as resolution scale based on the signal to noise ratio. Like many other radar applications, the definition of resolution is changed due to the application function. In this research, we are extracting weak target features by increasing the Radar aperture length modeling dominant scatterer as extra transmitter. The simulated results are shown image exceeding $\lambda / 50$ resolution. 


\subsection{Resolution Limits}

Resolution is one of the most important parameters regarding a radar system, especially in considering imaging applications. Before discussing the radar super-resolution, a general background of resolution criteria is presented. Many properties of radar resolution are similar to the properties and criteria as for an optical system. This analogy helps to explain the limitations and improvements in radar imaging systems [60]. The most widely known criteria for describing resolution is the Rayleigh criterion. The Rayleigh resolution limit is defined by the distance between the central maximum and first minimum of the point spread function intensity in the image [61]. Two point spread functions in the criterion can be replaced by two point sources to define the resolution limits as the distance between the mainlobes of the point spread function as shown in Figure 7. Furthermore, the classical resolution criteria treat the point source as incoherence illumination. The radar tomographic system is modeled as coherent illumination point sources. Other criteria are proposed to deal with coherent two point resolution. Later partially coherent illumination was introduced by Born and Wolf that expanded the resolution limits criteria [34]. Developing a criteria to define single source resolution instead of two sources was intended to extend the resolution criteria to measure the performance of other radar applications than just a single object sensing. In this research, the radar system resolution is the main parameter of concern. The objective is to characterize the true resolution of a radar system. The single coherent source resolution is defined as the ability of the system to locate and predict the point spread function contained within a noisy environment. The noise, in this case, can be either background Gaussian White noise or colored Gaussian noise from other 
targets sidelobes. The resolution criteria has been examined and improved with sophistication over growing applications. Moreover, the enhancements to the accuracy of modeling the point spread function and the transfer function sufficiently has also resulted in lowering of the artifact. Modeling dominant scatterer as dipole improves the point spread function also resulting in lowered artifact. The measurement errors have been taken into account since the classical criteria are measuring the resolution limits for calculated images with noise free models. The target feature will be revealed once the noise is reduced and improve the point spread function modeling. Considering the signal-to-noise ratio effects on the resolution scale was proposed in radar theory by Dunn and Howard [62]. Falconi found that the relationship between Rayleigh limit and signal-to-noise ratio is a function of the mean square and the angular resolution in radar system and can be adapted for optical purposes. Harris proposed the separation space exceed Rayleigh limit when the signal-tonoise ratio is large. Single source resolution scale was derived by Fried to scale the resolution of an optical system. Idell and Webster introduced new resolution limits based on SNR measurement which can represent the resolution in form of SNR. Note that the adoption of the SNR as parameter to scale the resolution was introduced first in radar system then it was adapted for single source resolution scale in optical system. For radar imaging purposes, we have to have a scale that works for single point source imaging where Rayleigh limit is not presenting the resolution for single target or target's features. This research is aimed to improve the separation of weak scatterer and dominant scatterer. The results will be similar to a form of SNR proposed by Idell and Webster. The concept of super-resolution will be defined in next chapter. 


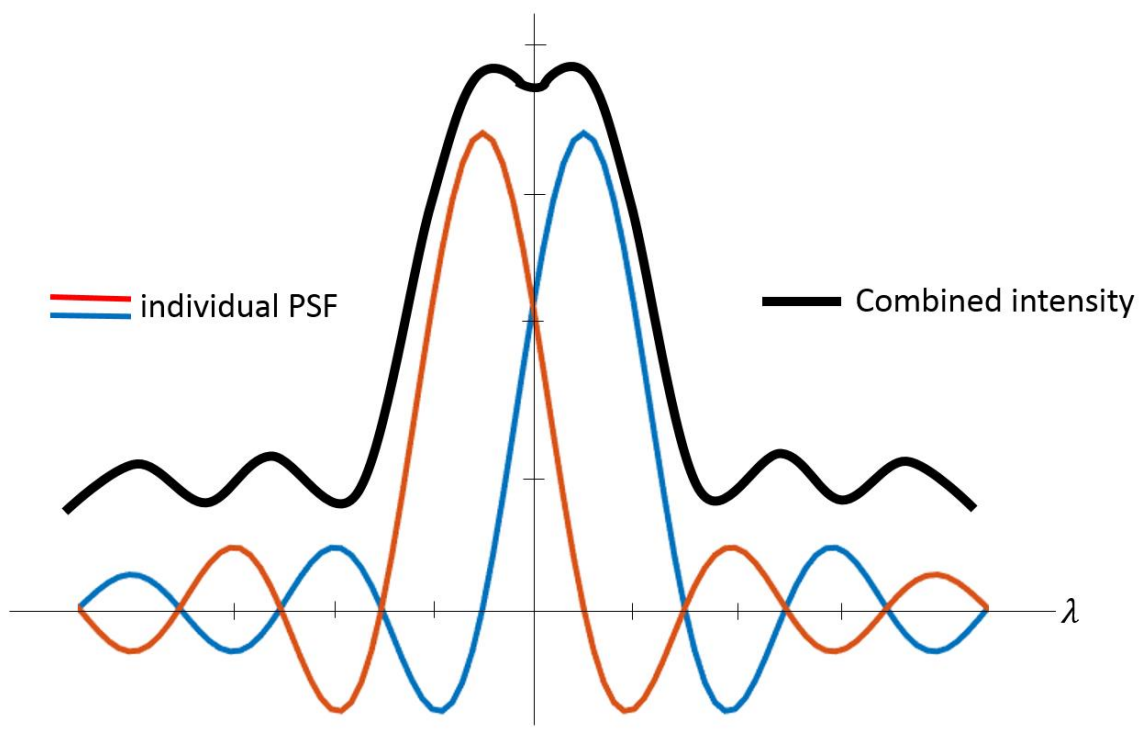

Figure 7: Closely spaced targets with the same intensity.

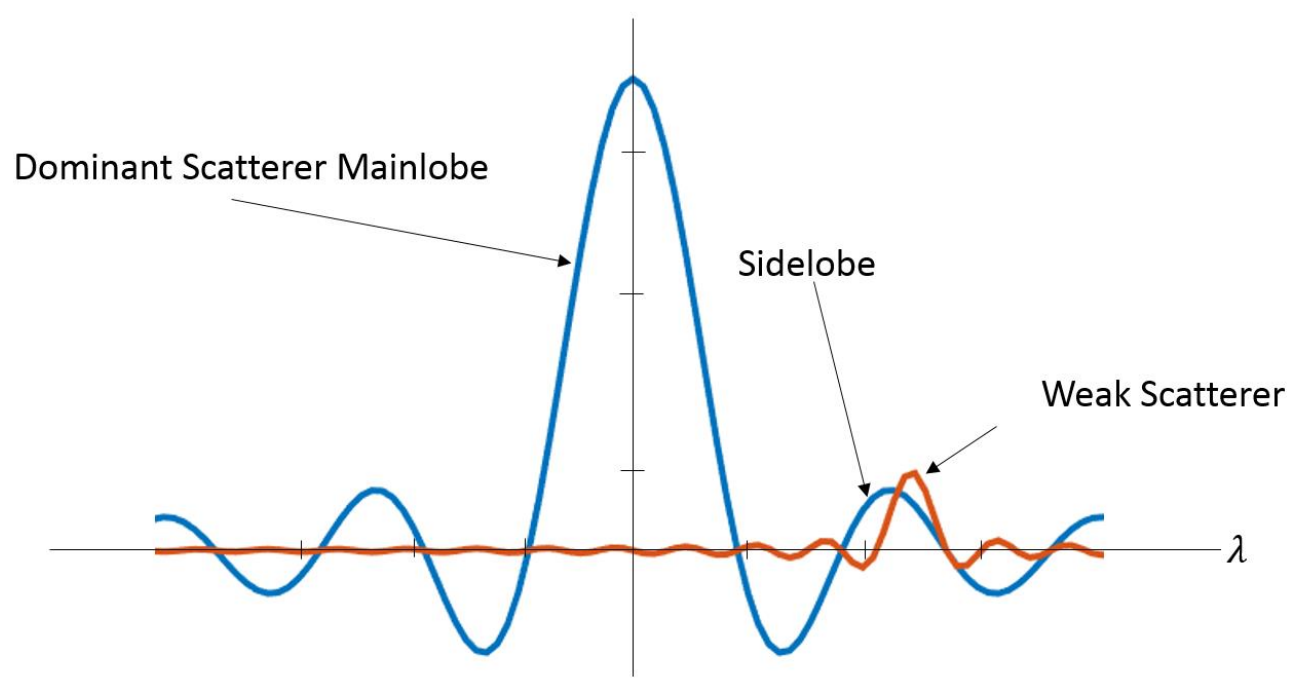

Figure 8: Dominant scatterer sidelobe masks weak scatterer.

\subsection{Super-resolution in Radar Systems}

In previous to be addressed in this research is not a typical resolution problem that can be represented in separation form. The Rayleigh limits, defined as the separation distance between two sinc functions, is shown in Figure 7. For human vision, resolution is defined 
as the ability to distinguish separation of two objects. But in this research the separation and the intensity of the sinc function is critical. The ideal form of the problem was previously stated in the introduction. The underlying assumption is that for two objects, one of them is the dominant scatterer while the other is the weak scatterer. The point spread function of the dominant scatterer sidelobe masks the weak scatterer as shown in Figure 8. The proposed solution is to model the dominant scatterer as an extra transmitter and predict the phase and magnitude as the polarization of this extra transmitter. This technique results in an improved model of the dominant scatterer point spread function. Super-resolution occurs when the Rayleigh limits are exceeded. Better resolution will be obtained among the measurement errors and the system model accuracy. We will use the SNR resolution scale for the Radar imaging system. The resolution scale characterizes the Radar image in the frequency domain to present the SNR of the Fourier spectrum of the Radar image. The contrast function (image) of the measurement domain is giving by $\tau_{\delta}\left(\mathbf{r}^{\prime}\right)$. The twodimensional image plane coordinate is represented by $\mathbf{r}^{\prime}=(x, y)$, the equivalent twodimensional spatial frequency coordinate is donated by $\mathbf{f}=\left(f_{x}, f_{y}\right)$. The Fourier transform of the contrast function is denoted by $\xi(\mathbf{f})$. The SNR of the contrast function in frequency domain is given by

$$
\operatorname{SNR} D(\mathbf{f})=\frac{|\mathrm{E} \xi(\mathbf{f})|}{\sqrt{\operatorname{Var} \xi(\mathbf{f})}}
$$

Targets with low SNR have more probability to be masked under the dominant scatterer sidelobe. As a result, the brightness will be degraded, and speckle artifacts occur due to the sidelobe effects. Knowing the noise mechanism in the frequency domain improves the 
estimation of the point spread function of the weak scatterer and displays the preform of Radar imaging system. Using the first image as a reference image, the minimum $\mathrm{SNR}_{\mathrm{M}}$ occurs at the highest frequency by (44). Then the proposed algorithm is applied to suppress the sidelobe of the dominant scatterer and exploit the multipath effects using the dominant scatterer as extra transmitter in the measurement domain. Applying equation (44) again to the final image after the algorithm process finds the $\mathrm{SNR}_{\mathrm{M}}$ that can be used to find the maximum frequency as shown in the following equation.

$$
\min \left(\mathrm{SNR}_{\mathrm{M}}\right) \rightarrow \max \left(f_{M}\right)
$$

The highest frequency obtained from the lowest SNR will determine the resolution as

$$
R=\frac{2 \pi f_{M}}{\lambda},
$$

Where $\lambda_{\circ}$ is the wavelength of the operation frequency of the radar system. The SNR of a cross range of weak scatterer image is shown in the next chapter to show the improvement in the image performance. 


\section{CHAPTER 6}

\section{SIMULATION AND RESULTS}

Simulations using the computational electromagnetic software FEKO@ package (Altair)

were performed to obtain the scattered field $\mathbf{E}^{\mathrm{S}}$ for three different scenarios. The RF tomographic imaging principles were applied to reconstruct the contrast function of the measurement domain of these scenarios to validate the proposed method. Preliminary simulation results, obtained by computing the diffracted fields with FEKO, indicated that it is possible to reconstruct an image.

\subsection{Near-field Point Spread Function of Single Target}

For the simulation, a domain containing two targets was considered. One target was a cylinder of $\lambda / 4$ radius placed in the center and the second target was a cylinder with $\lambda / 50$ radius placed at a distance of $5 \mathrm{~cm}$ along the $\mathrm{x}$-axis as shown in Figure 9. The first target being larger than the second target is considered to be the dominant scatterer to be incorporated into the forward model. If the dominant scattered remains within the scene, then the reconstructed image will have sidelobes that mask the weak target response, as shown in Figure 10. 
After determining the location of the dominant scatterer, the eigenvalue and eigenvector analysis was carried out to determine its phase and magnitude as shown in Figure 11. The dominant scatterer was then replaced with a dipole that has the same electromagnetic effect, thus acting as an additional transmitting source (see Figure 12). The extra transmitter will remove the sidelobes of the dominant scatterer and improves the PSF of the remaining target in the scene. When the additional transmitter is added to the equation, a new image can be formed, as shown in Figure 13. The exploitation of the dominant scatterer as a source of transmission completely removes the sidelobes resulting in enhanced visibility of the weak targets; This is more suitable for classical detection algorithms.

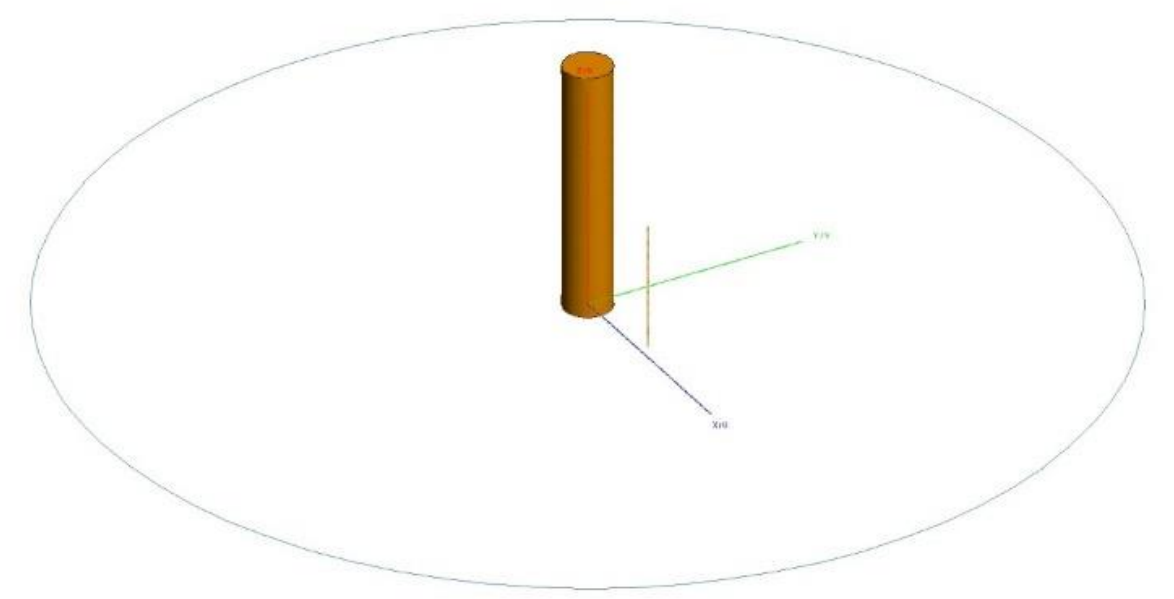

Figure 9: Two targets: a cylinder with radius $\lambda / 4$ located in the center and a cylinder with radius $\lambda / 50$. 


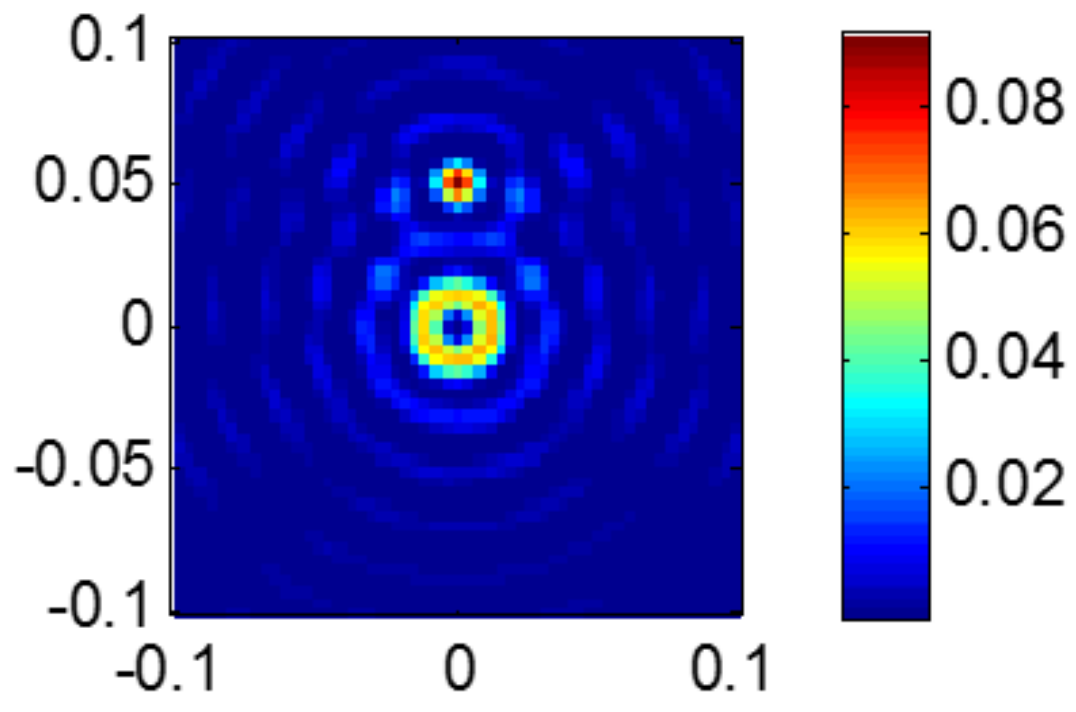

Figure 10: The resulting of conventional RF tomographic image of two targets.

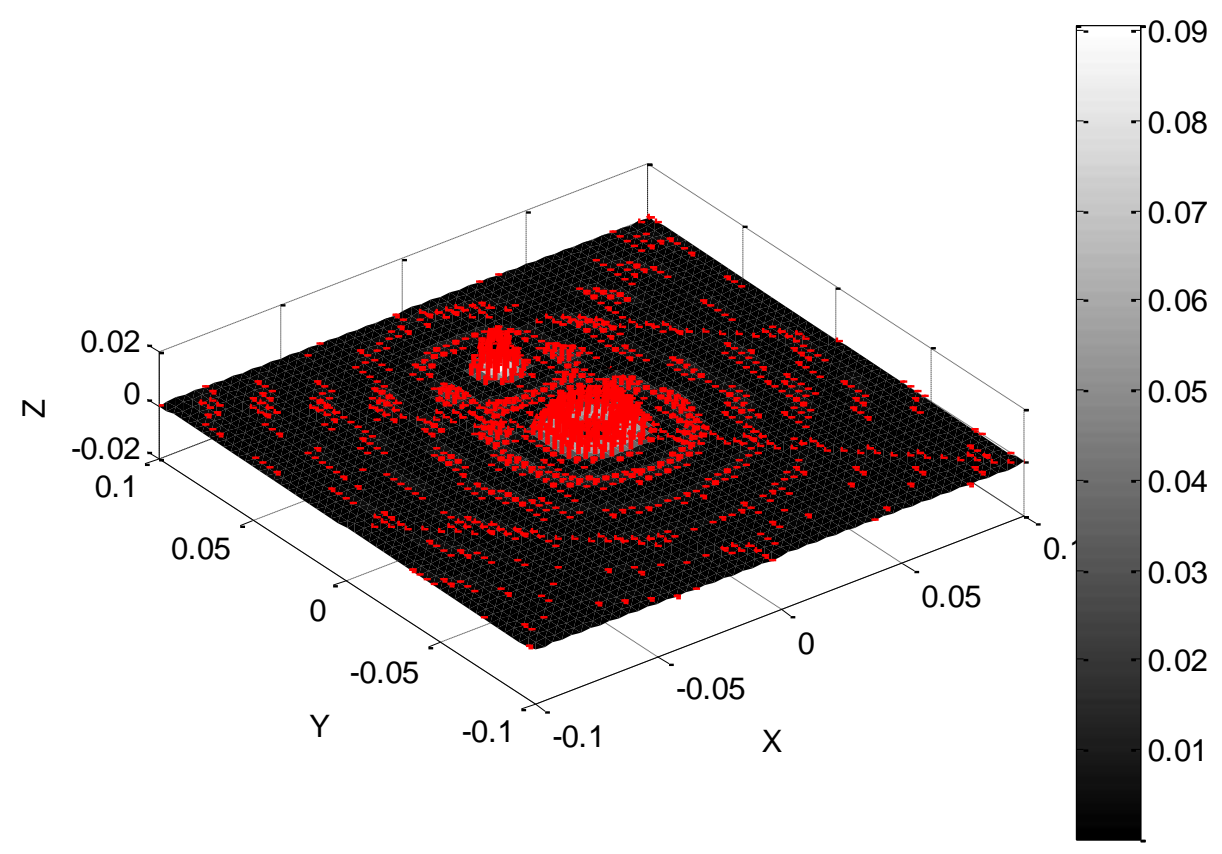

Figure 11: The conventional RF tomographic image is represented as cells vector of the two targets. 


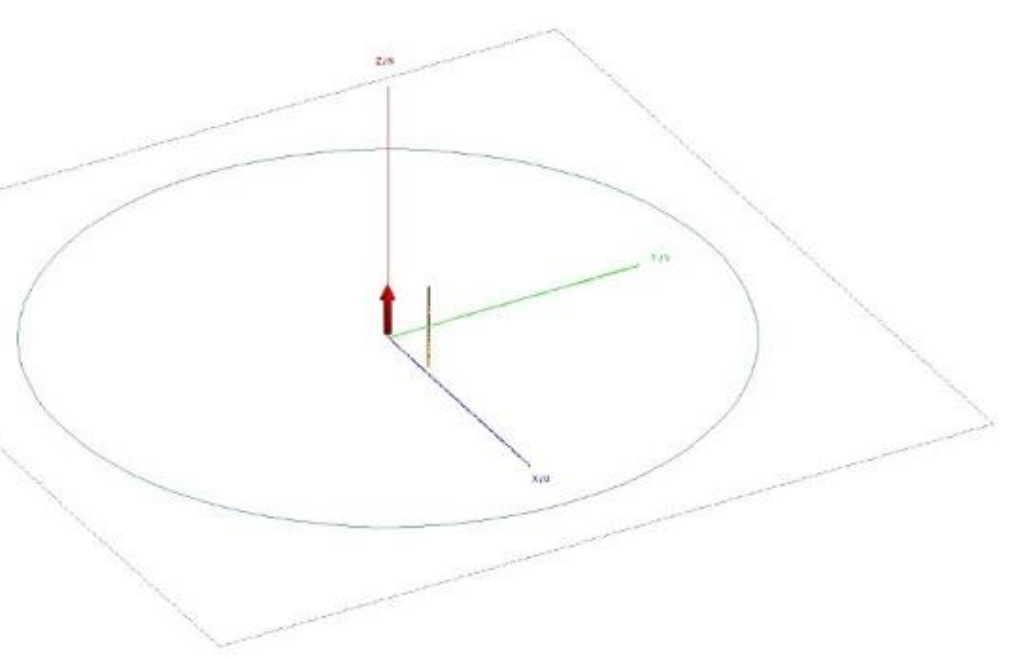

Figure 12: The dominant scatterer assumed to be a dipole.

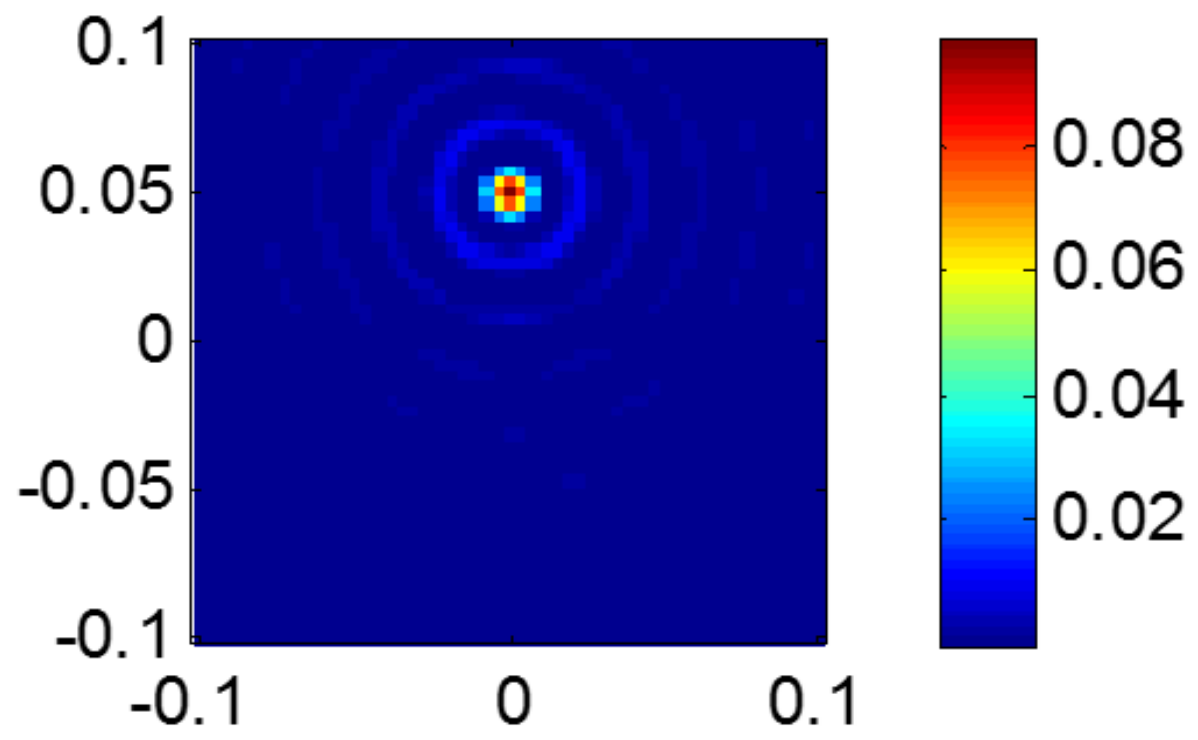

Figure 13: Domain after being replaced by the dominant scatterer with a dipole. 


\subsection{Near-Field Complex Targets}

Designing PSF for a single target was exploit and presented previously. In this section the PSF of a complex target at near-field region is performed and tested to expand the simulation results. The complex target can be a set of simple targets in regular and irregular geometries.

\subsubsection{Regular Geometry}

The operating frequency is $8 \mathrm{GHz}$, and the measurement domain used is $0.25 \mathrm{~m}$ by $0.25 \mathrm{~m}$ divided into an array of $133 \times 133$ cells of dimension $0.1 \lambda \times 0.1 \lambda$. The plane of the two-dimensional cut was simulated at $z=0$. The simulation used 19 transmitters placed at the center of a $2 \mathrm{~m}$ radius circle within the measurement domain. 51 receivers were placed in a concentric circle with a radius of $0.4 \mathrm{~m}$. A domain containing 12 cylinders, forming a complex target, was considered, as illustrated in Figure 14. The cylinders were aligned with the $\mathrm{z}$-axis and are uniformly spaced into an L-shape along the $x$ and $y$ directions. These cylinders have a radius of $\lambda / 4$, a length of $0.05 \mathrm{~m}$ and center-to-center spacing of $\lambda$ between adjacent cylinders. A single weak scatterer is modeled as an additional cylinder, with a radius of $\lambda / 50$ and located at coordinate $(0.1 \mathrm{~m}, 0.112 \mathrm{~m})$ in the $x y-p l a n e$, as shown in Figure 14. The 12 cylinder complex target is larger as compared to the small single cylinder. Therefore, it acts as a dominant scatterer in the simulation, and will mask the echo from the single weak scatterer. The forward model is applied to create simulated scattered field to begin the algorithm. The matrix $\mathbf{L}$ operator is $969 \times 17689$ elements representing the predicted electric field response under Born approximation. The first application of the $\mathbf{L}$ operator inversion leads to a reconstruction as shown in Figure 15. This correspond to 
[zz], in which the weaker scatterer does not appear. After determining the cell location of the dominant scatterer, the eigenvalue and eigenvector analysis was carried out to determine its phase and magnitude, as shown in Figure 17. The algorithm then proceeded, as previously described, by replacing the 12 dominant scatterers, one at a time, with modeled dipoles that have the same electromagnetic effect. After suppressing all of the dominant scatterers in the complex target, the final image produced by the algorithm is shown in Figure 21. The weak scatterer that did not appear in Figure 15 is now clearly visible in Figure 21 after applying Mumma Radar Laboratory (MRL) algorithm. The power density distribution of the measurement domain in Figure 16 shows the reflected power of the PSF of the 12 cylinders where its sidelobe mask the weak scatterer shown in Figure 18. The weak scatterer is very thin wire with radius $\lambda / 50$ made its PSF is very weak as shown in Figure 19 for $\mathrm{x}$-axis cross range and Figure 20 for y-axis cross range, respectively. The improvement in the image will be by maximize SNR of weak scatterer. Figure 22 shows the power distribution of the contrast function where the sidelobe was reduced using MRL algorithm. The close view of the PSF function in $\mathrm{x}$-axis and y-axis are shown in Figure 23 and Figure 24, respectively. 


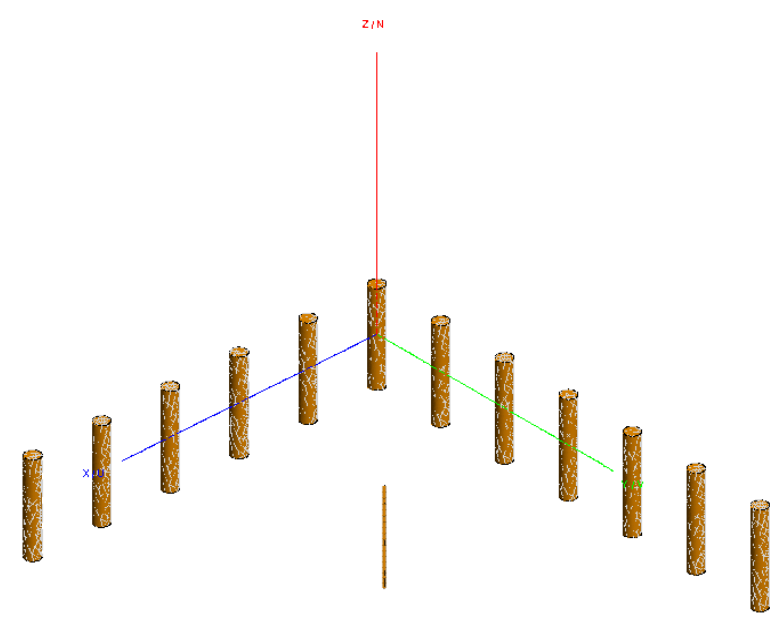

Figure 14: Thirteen targets: twelve cylinders with radius $\lambda / 4$ located in the center as L-shape complex target and a cylinder with radius $\lambda / 50$.

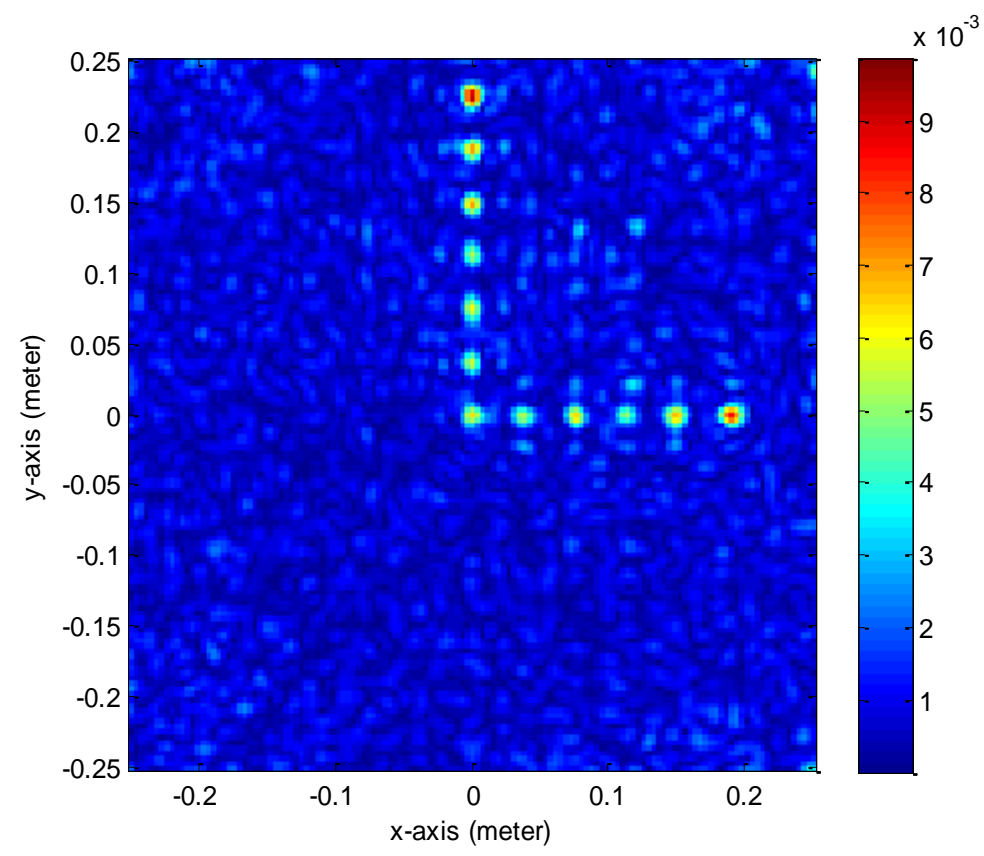

Figure 15: Initial tomographic image reconstruction, showing only the dominant target. 


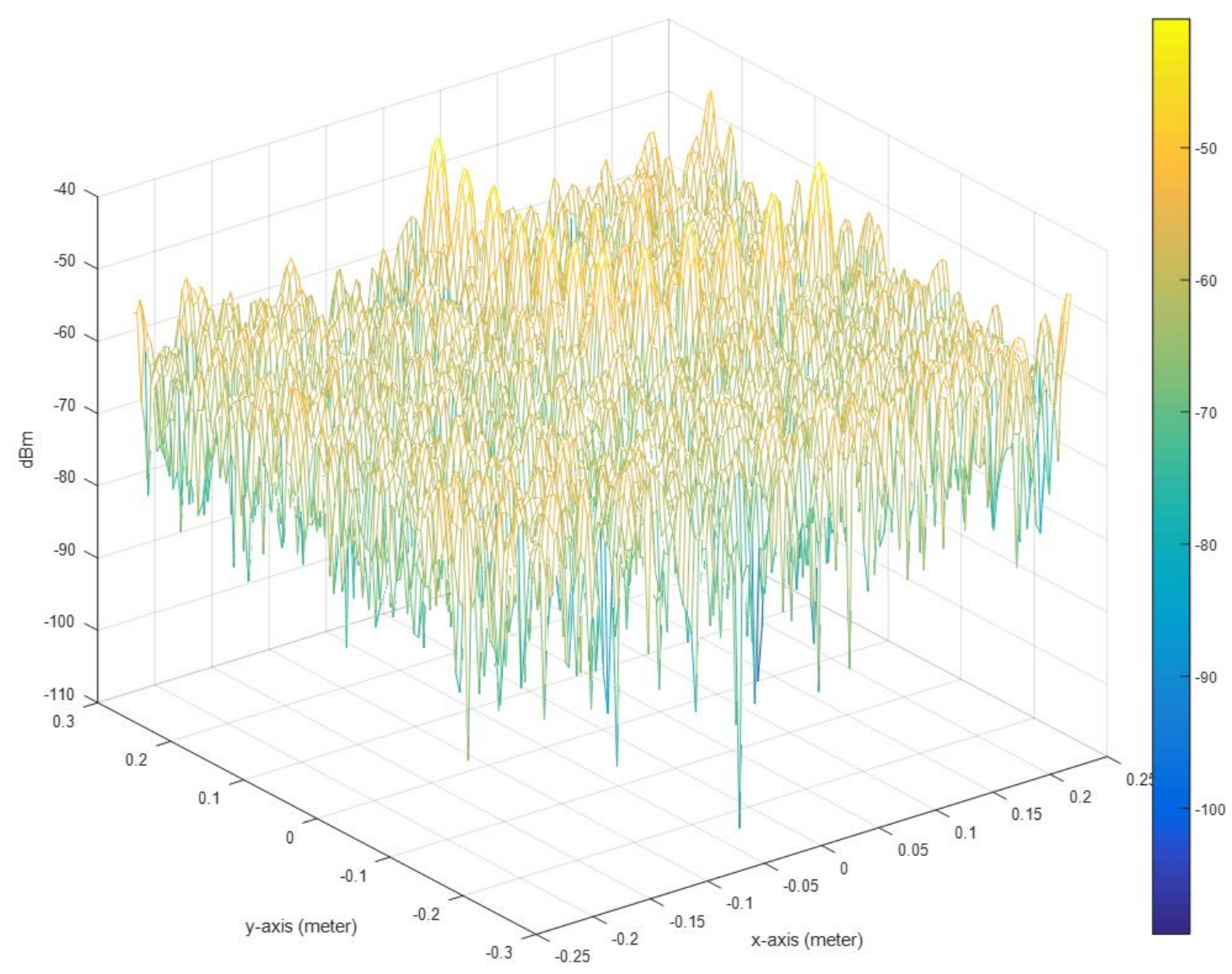

Figure 16: Intensity 3D analysis for the contrast function before applying MRL algorithm.

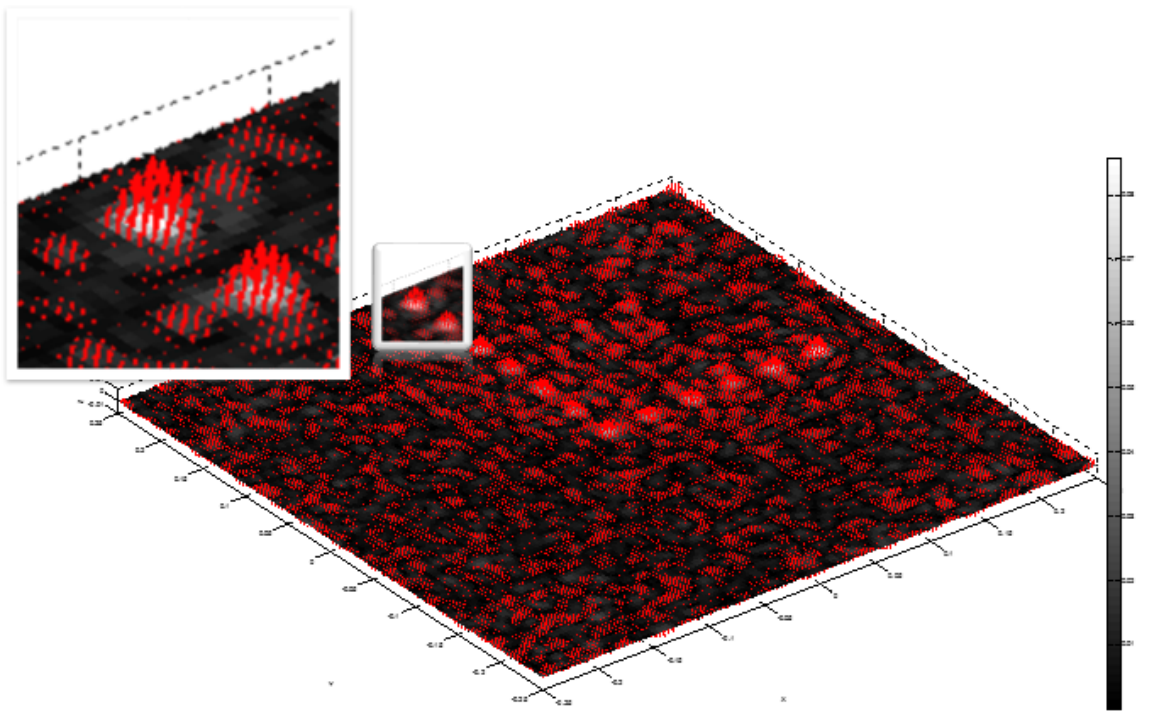

Figure 17: Initial tomographic image reconstruction represented as eigenvalues/eigenvectors. 


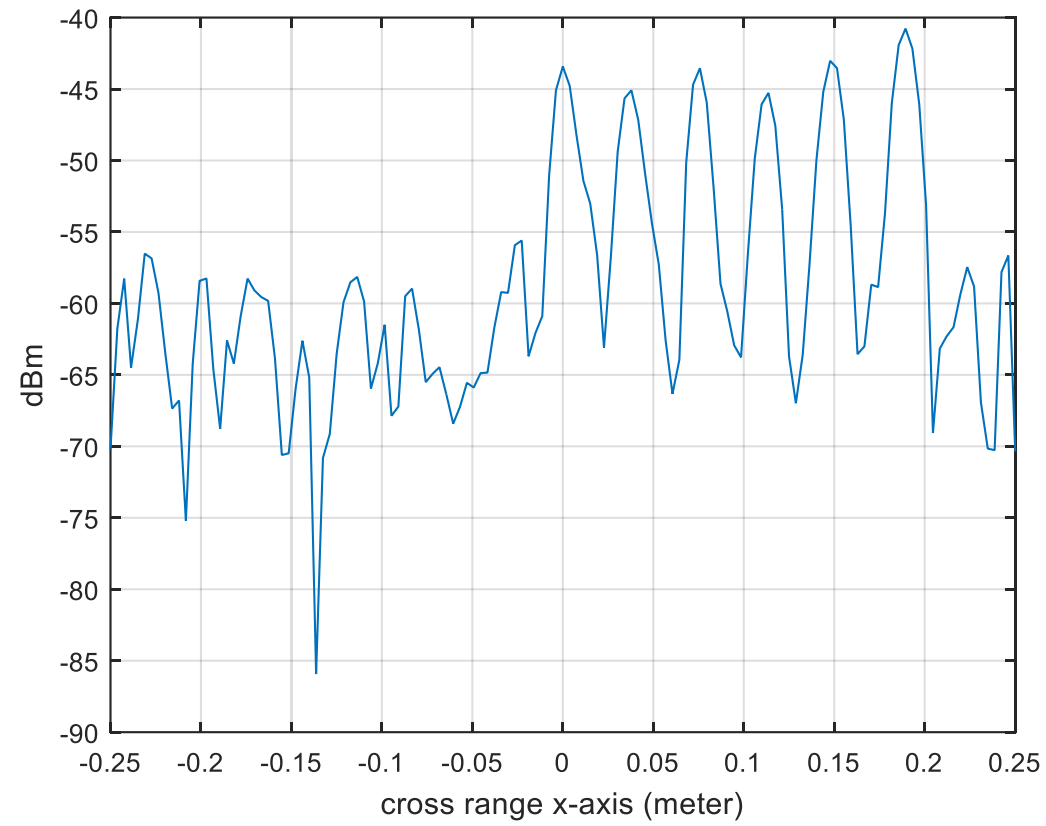

Figure 18: The cross range of contrast function intensity of mainlobe of six cylinders at $x$-axis.

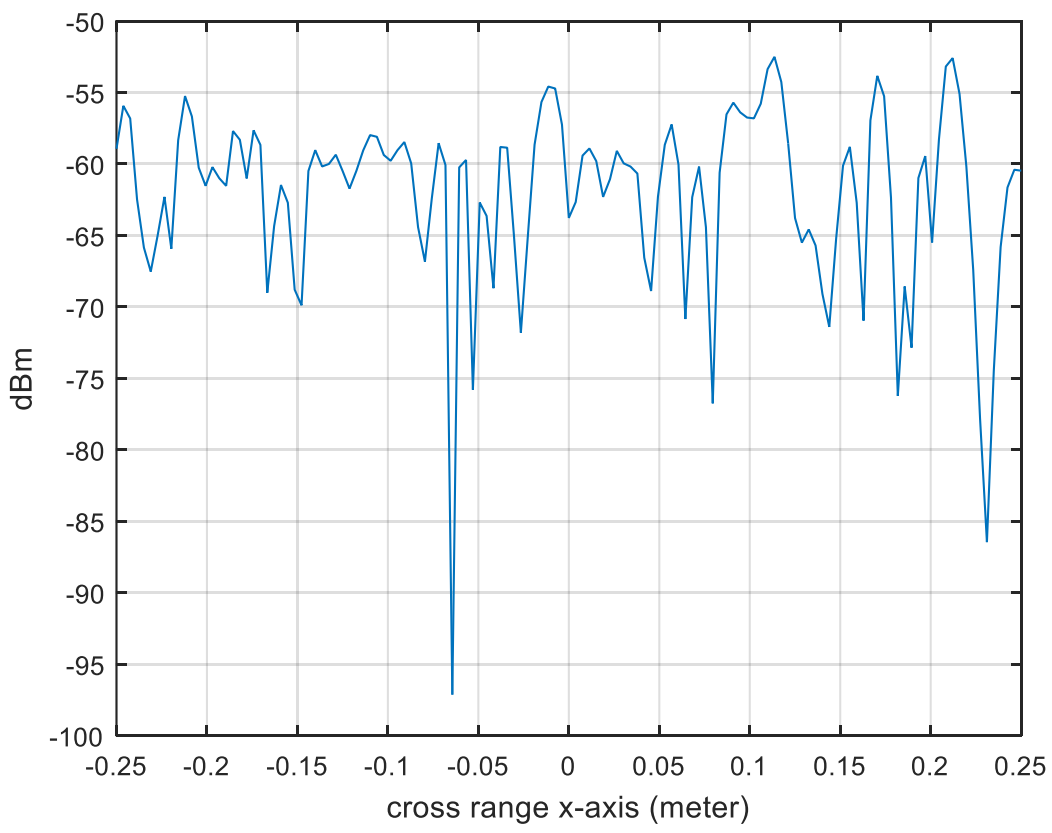

Figure 19: The intensity of the weak scatterer at the cross range $x$-axis of the weak scatterer. 


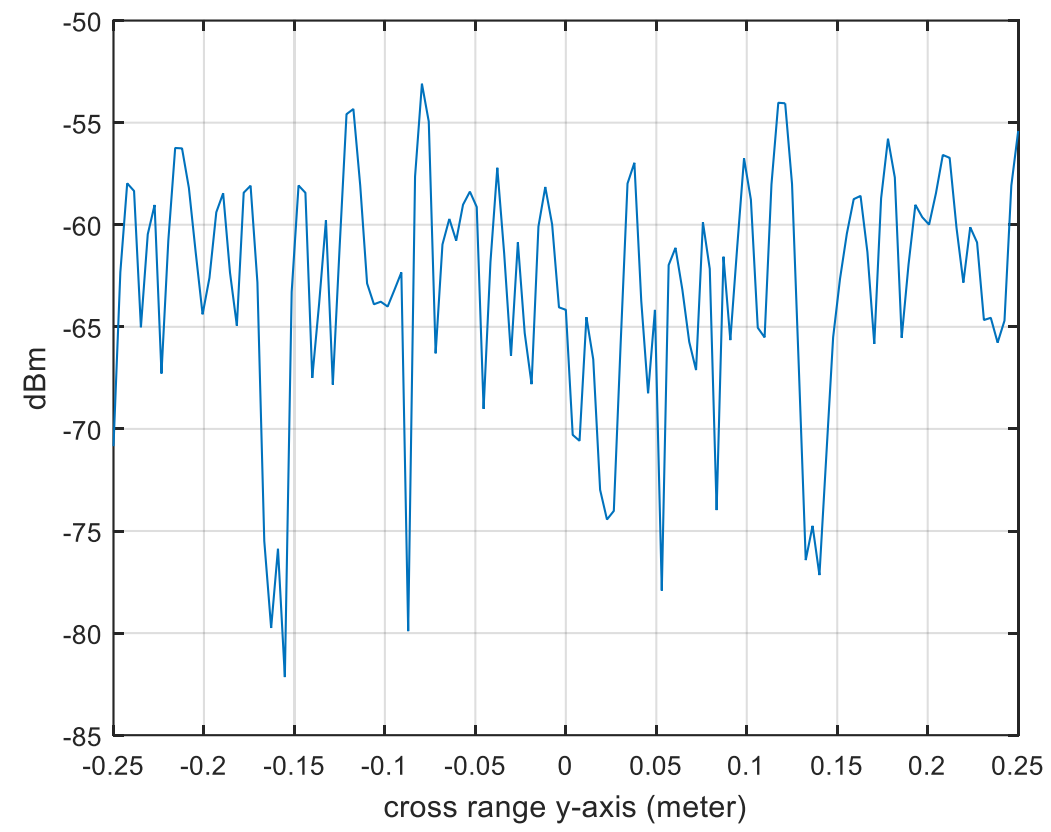

Figure 20: The intensity of cross-section of weak scatterer at y-axis.

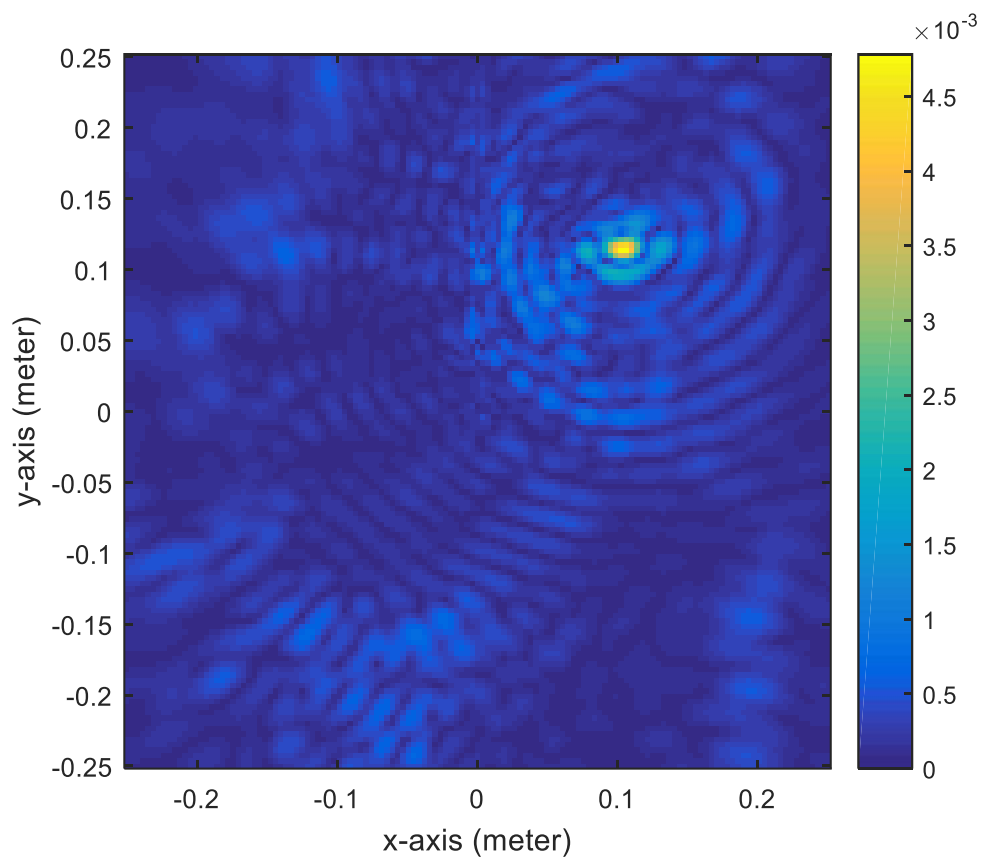

Figure 21: Final tomographic image reconstruction produced by the algorithm, isolating the effect of the weak scatterer. 


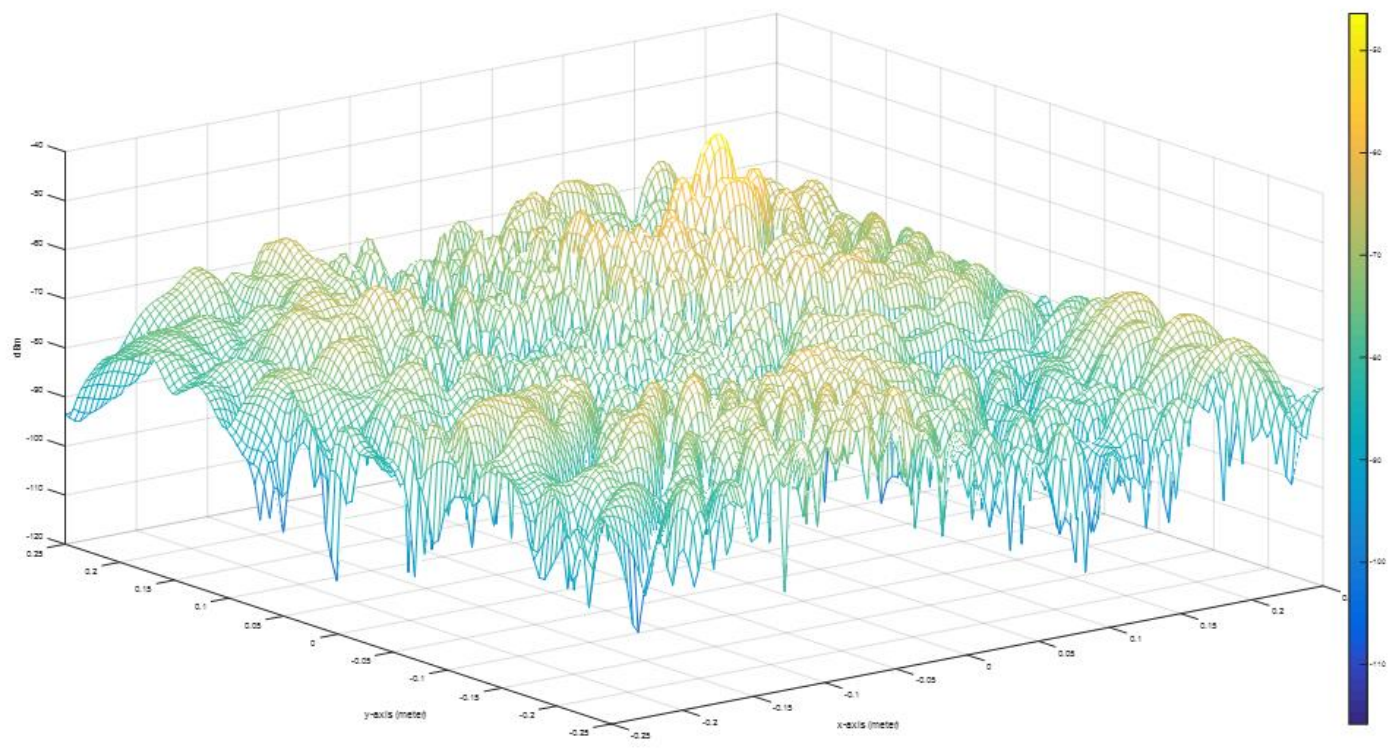

Figure 22: 3D intensity distribution analysis for the contrast function after applying MRL algorithm.

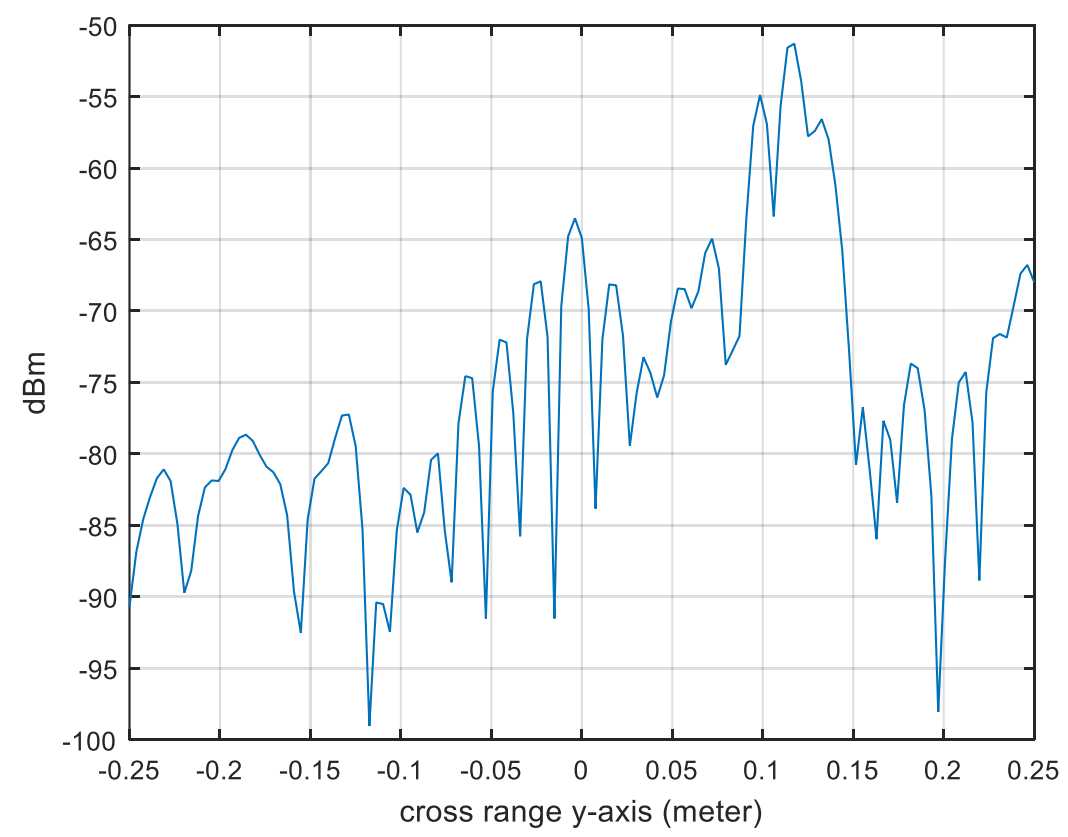

Figure 23: The sectional view of intensity distribution and sidelobe reduction at y-axis after applying MRL algorithm, weak scatterer at $0.112 \mathrm{~m}$. 


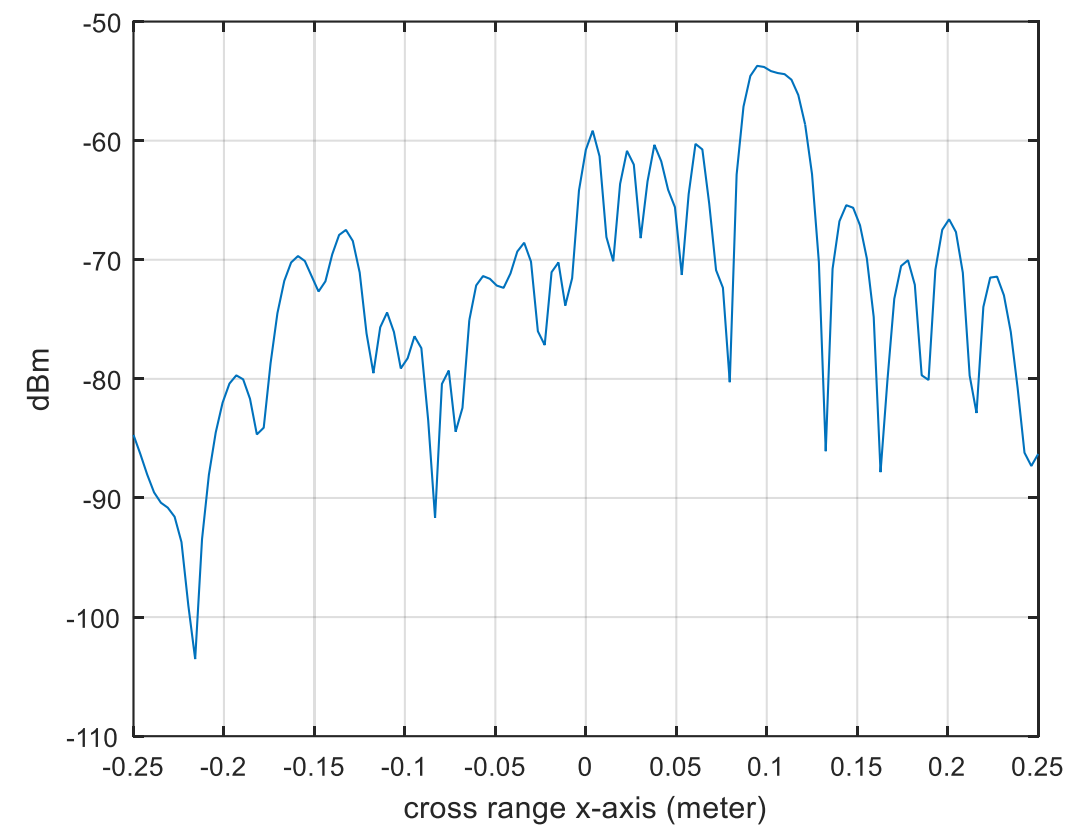

Figure 24: The sectional view of power density and sidelobe reduction at x-axis after applying MRL algorithm, weak scatterer at $0.1 \mathrm{~m}$.

\subsubsection{Irregular Geometry}

The simulation has done using the same transmitters and receivers parameters of previous simulation for regular geometry at $8 \mathrm{GHz}$ operating frequency. The measurement domain used is $50 \times 50 \mathrm{~cm}$ divided into cells of size $0.1 \lambda \times 0.1 \lambda$. The two-dimensional tomographic image was simulated at $\mathrm{z}=0$. The domain still has 12 cylinders placed randomly on the measurement domain creating irregular complex target geometry. Theses cylinders have radius of $\lambda / 4$, a length of $0.05 \mathrm{~m}$ as shown in Figure 25. The weak scatterer has radius of $\lambda / 50$ and still located at coordinate $(0.1 \mathrm{~m}, 0.112 \mathrm{~m})$ in the xy-plane. The initial tomographic image of contrast function and its and the power distribution of the measurement domain is shown in Figure 26 and Figure 27, respectively. Figure 28 and Figure 29 are the cross range view of the weak scatterer signal at $x$-axis and $y$-axis, which are showing its reflection buried under the sidelobe of the dominant scatterers. After 
applying MRL algorithm to process the domain scatterers as dipoles, the weak scatterer is reveal as shown in Figure 30 and its power distribution in Figure 31. The sidelobe reduction of the dominant scatterers shown better performance in sectional view of the weak scatterer at Figure 32 and Figure 33.

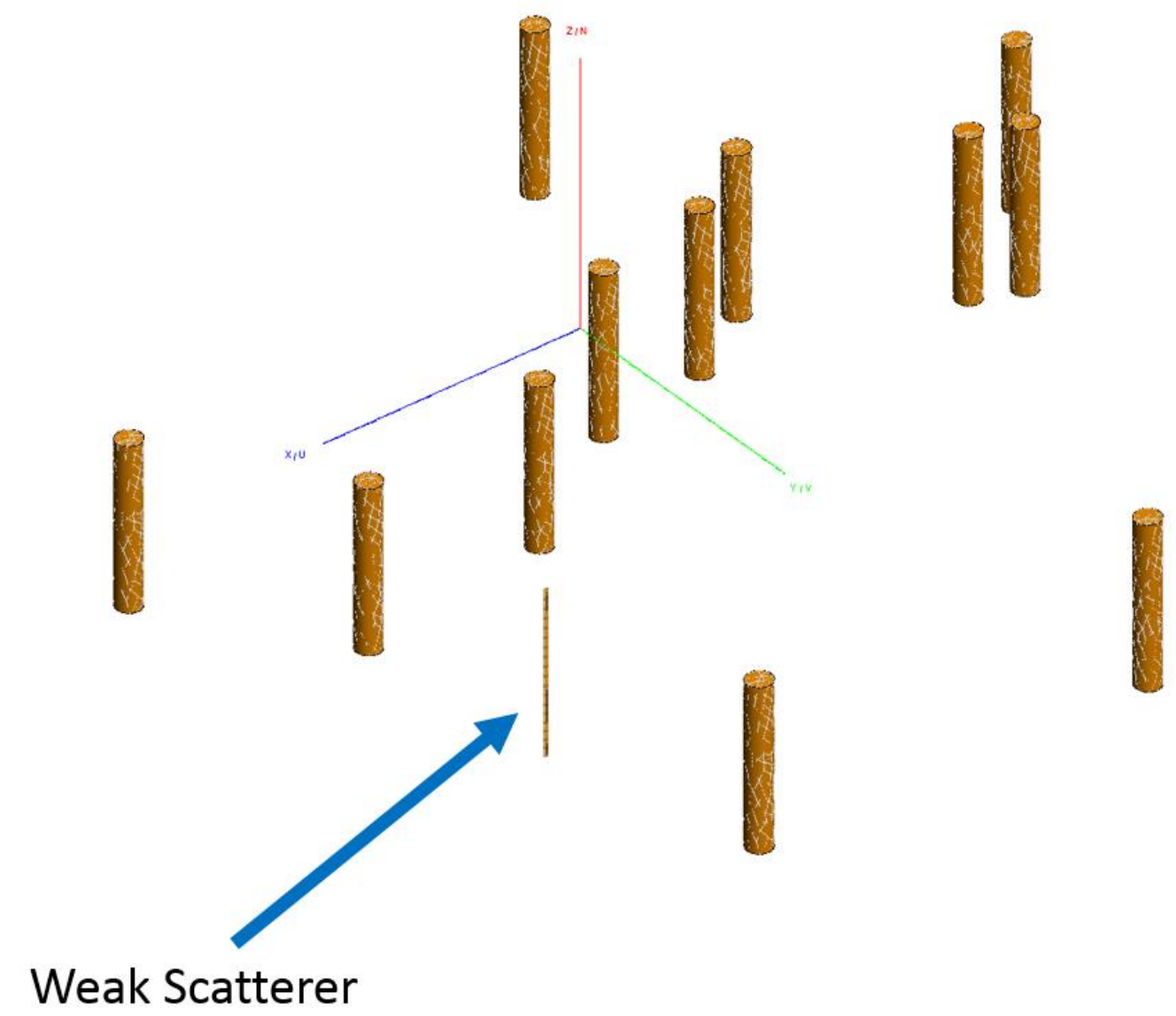

Figure 25: The random distribution of 12 cylinders creating complex target. 


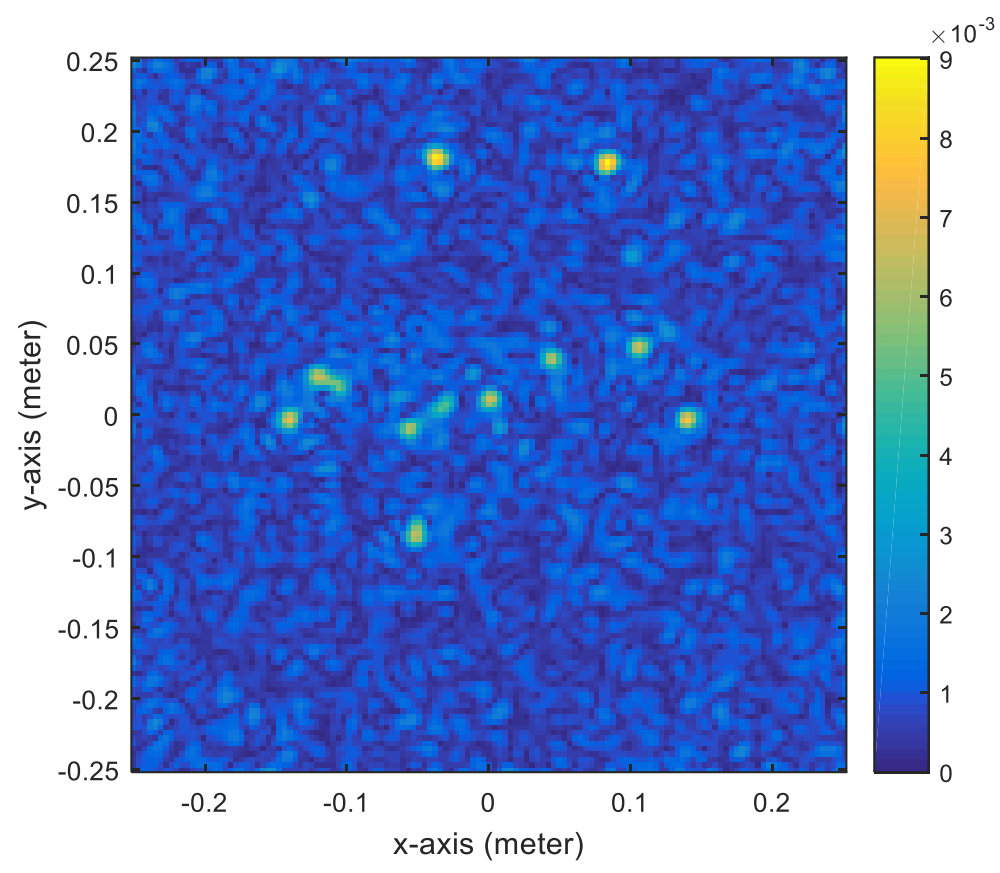

Figure 26 : Initial tomographic image reconstruction of irregular complex target, showing only the dominant target before applying MRL algorithm

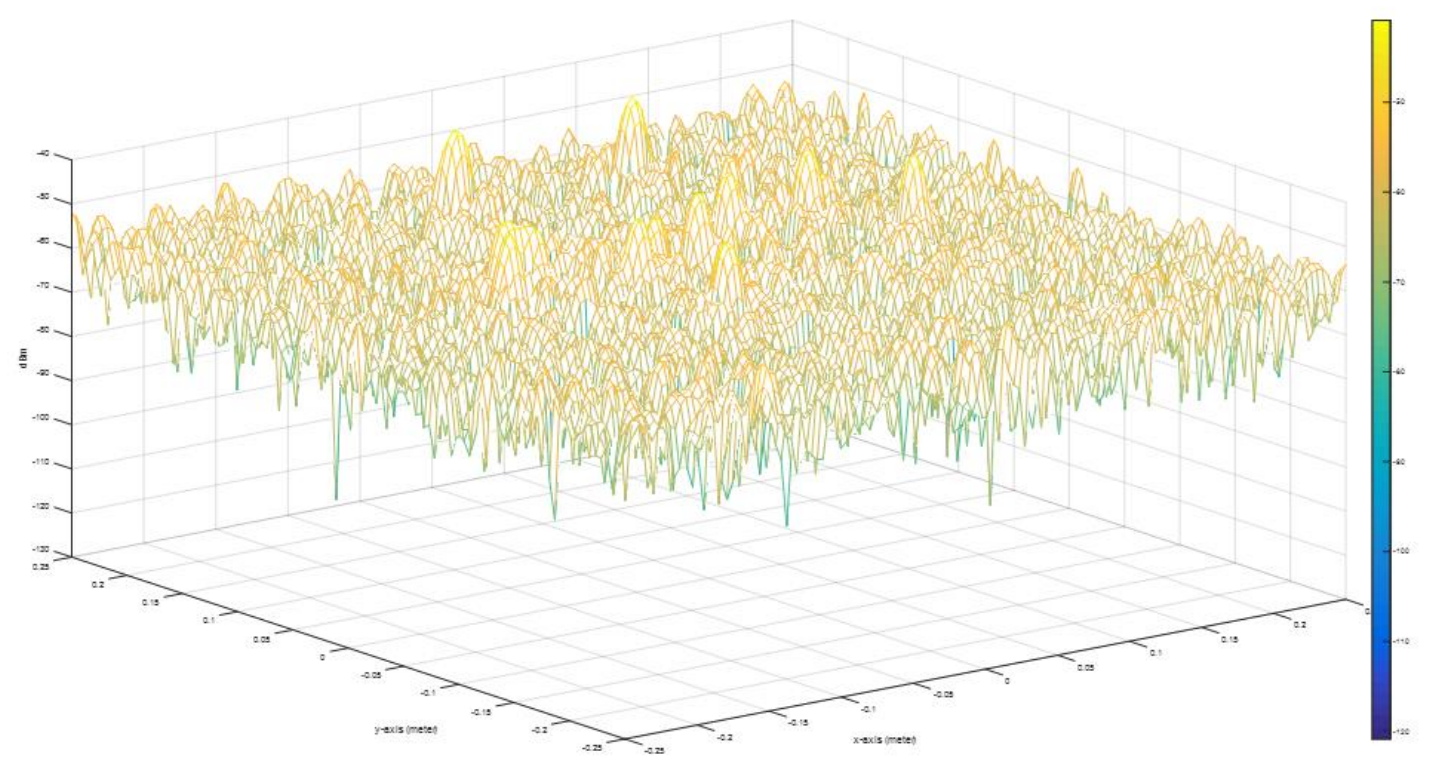

Figure 27: The intensity distribution of the contrast function, showing the reflected power over measurement domain before applying MRL algorithm. 


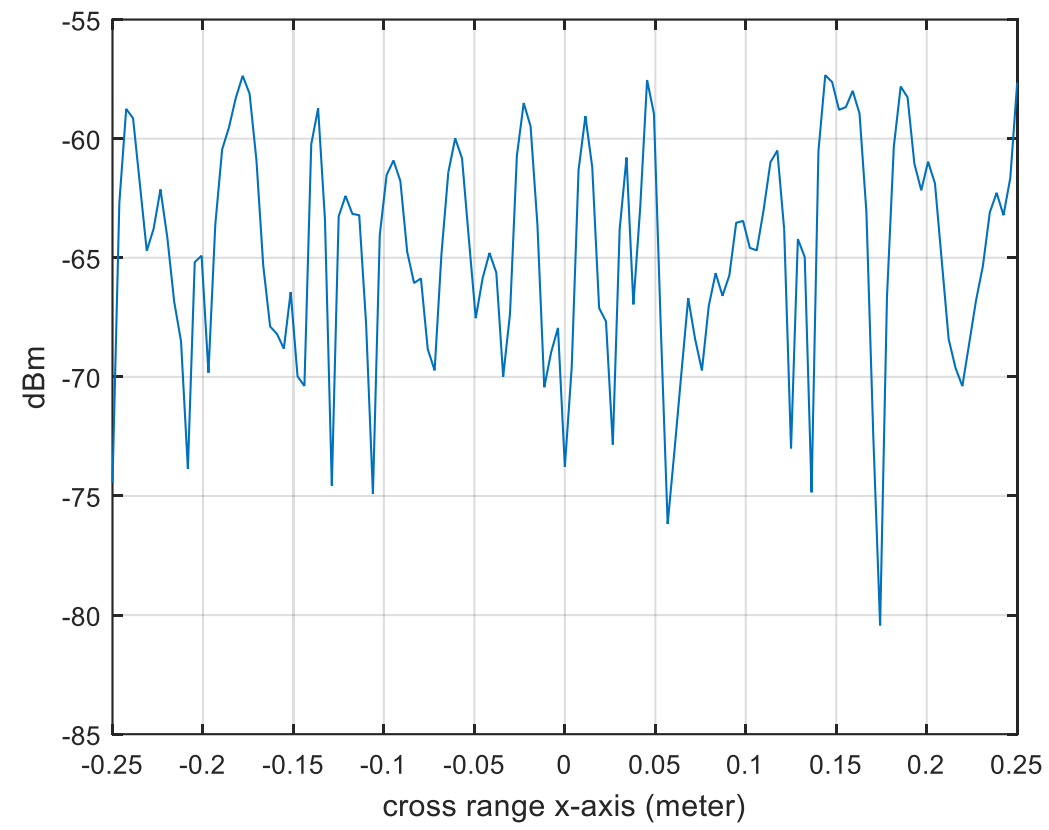

Figure 28: y-axis cross range of weak scatterer, showing the sidelobe suppressing weak scatterer signal before applying MRL algorithm.

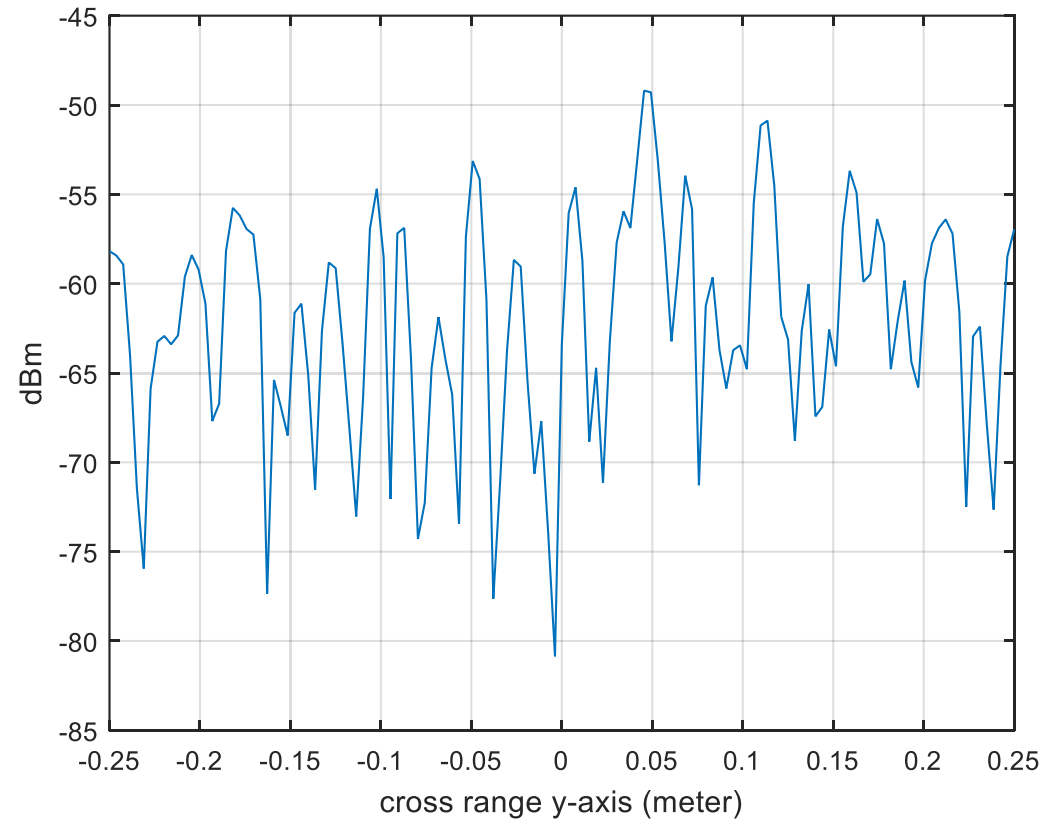

Figure 29: $y$-axis cross range of weak scatterer, showing the sidelobe suppressing weak scatterer signal before applying MRL algorithm. 


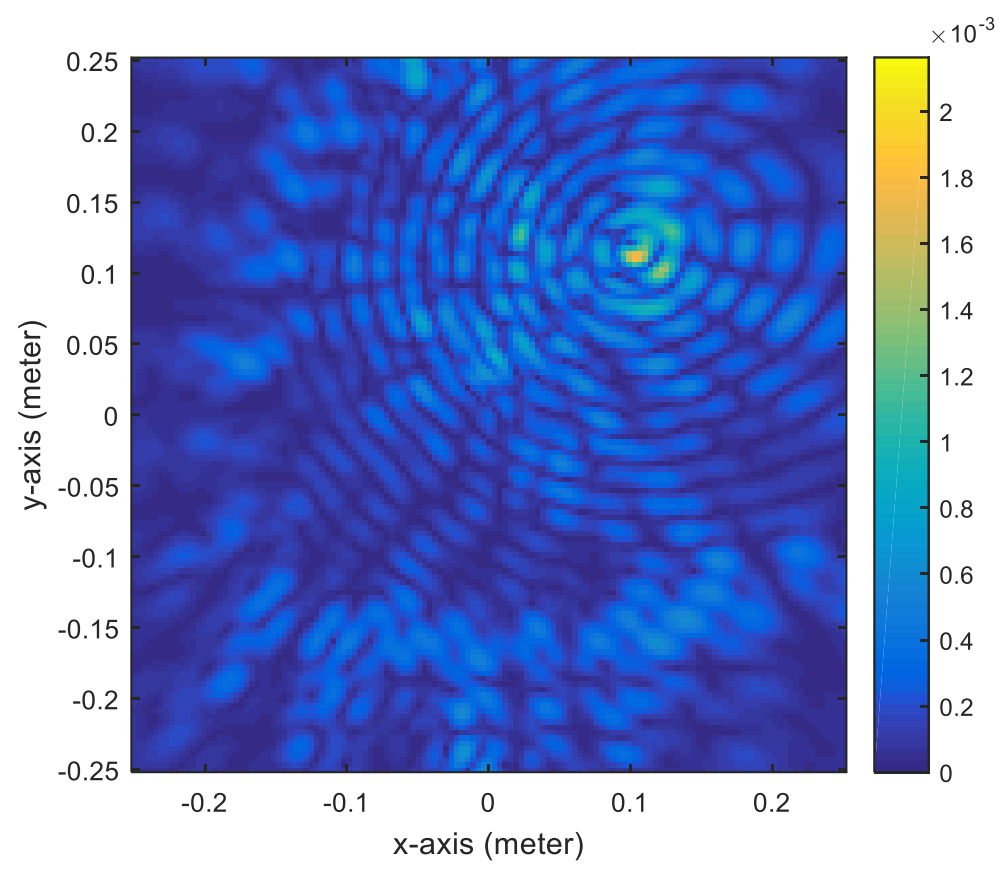

Figure 30: Final tomographic image reconstruction for irregular complex target geometry produced by the algorithm, isolating the effect of the weak scatterer.

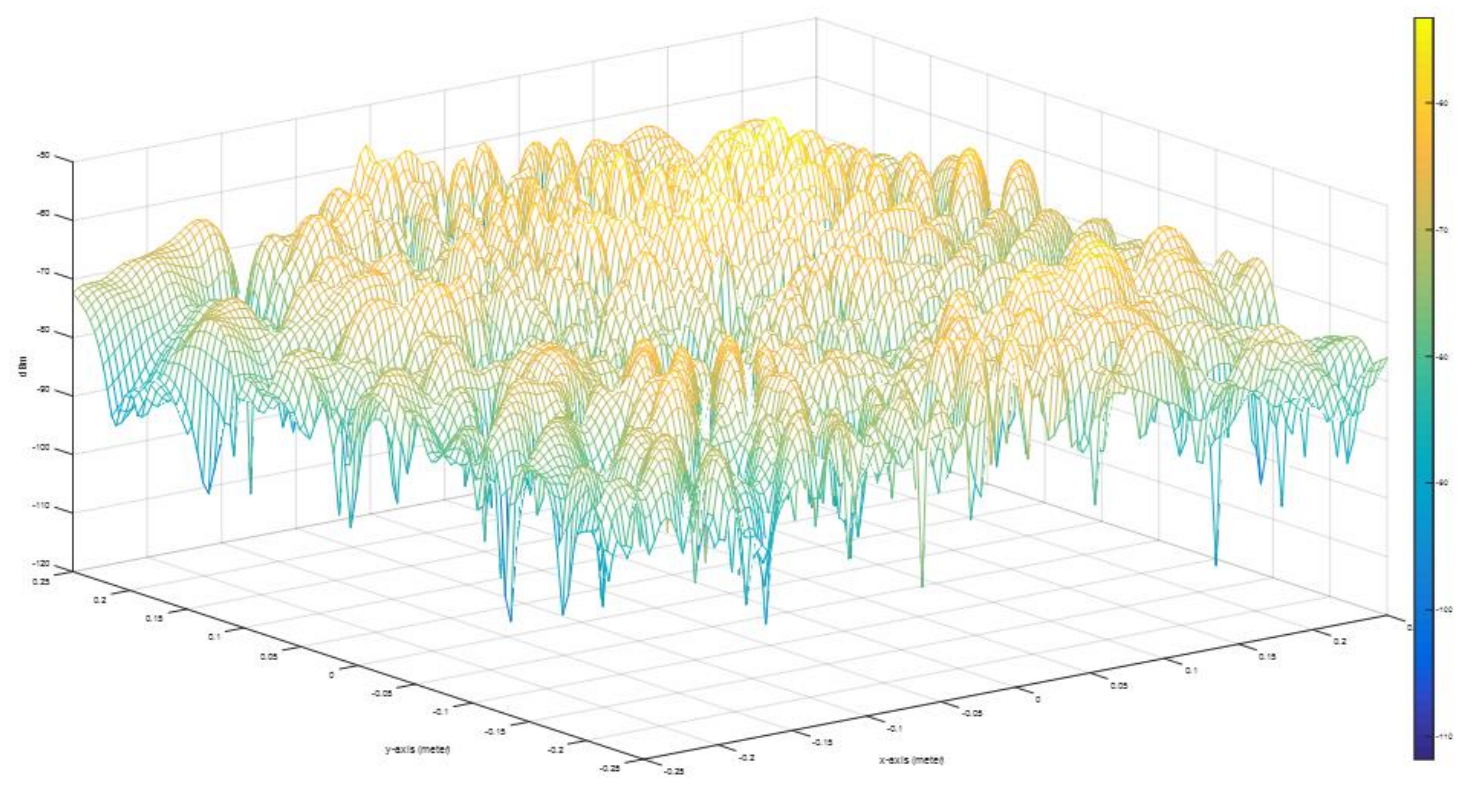

Figure 31: The power distribution of the contrast function, showing the reflected power over measurement domain after applying MRL algorithm. 


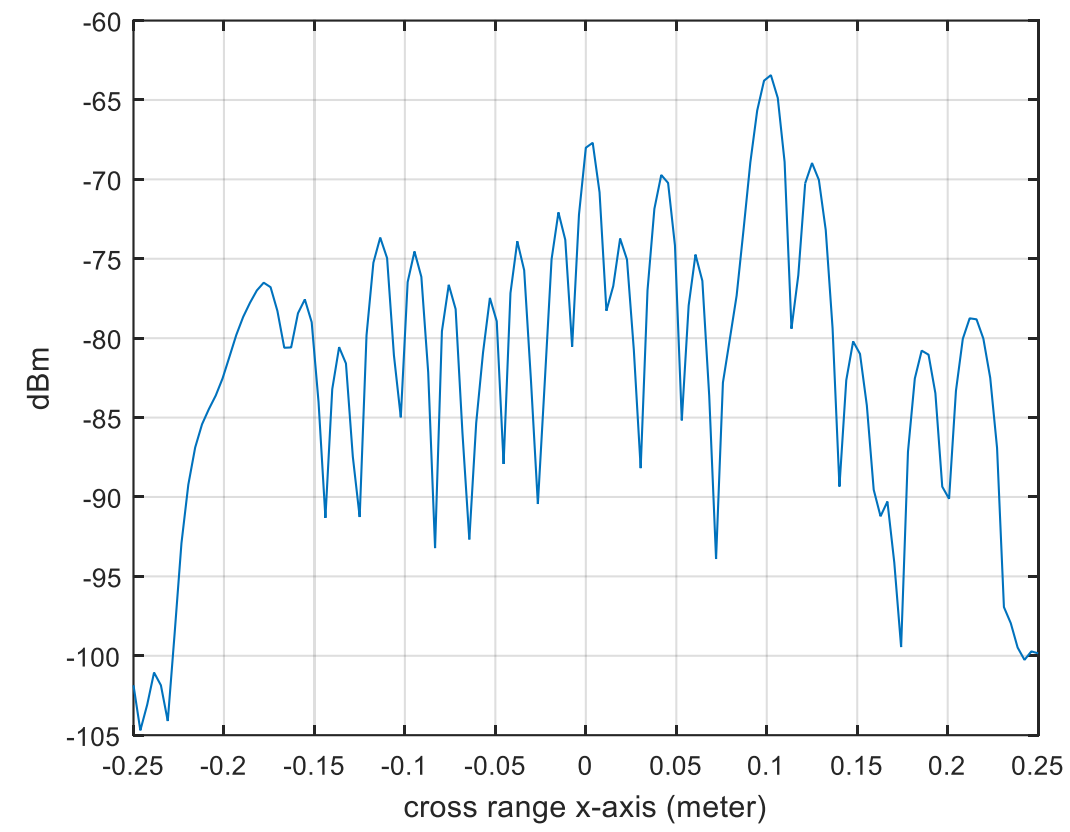

Figure 32: $x$-axis cross range of weak scatterer, showing the sidelobe suppressing weak scatterer signal after applying MRL algorithm.

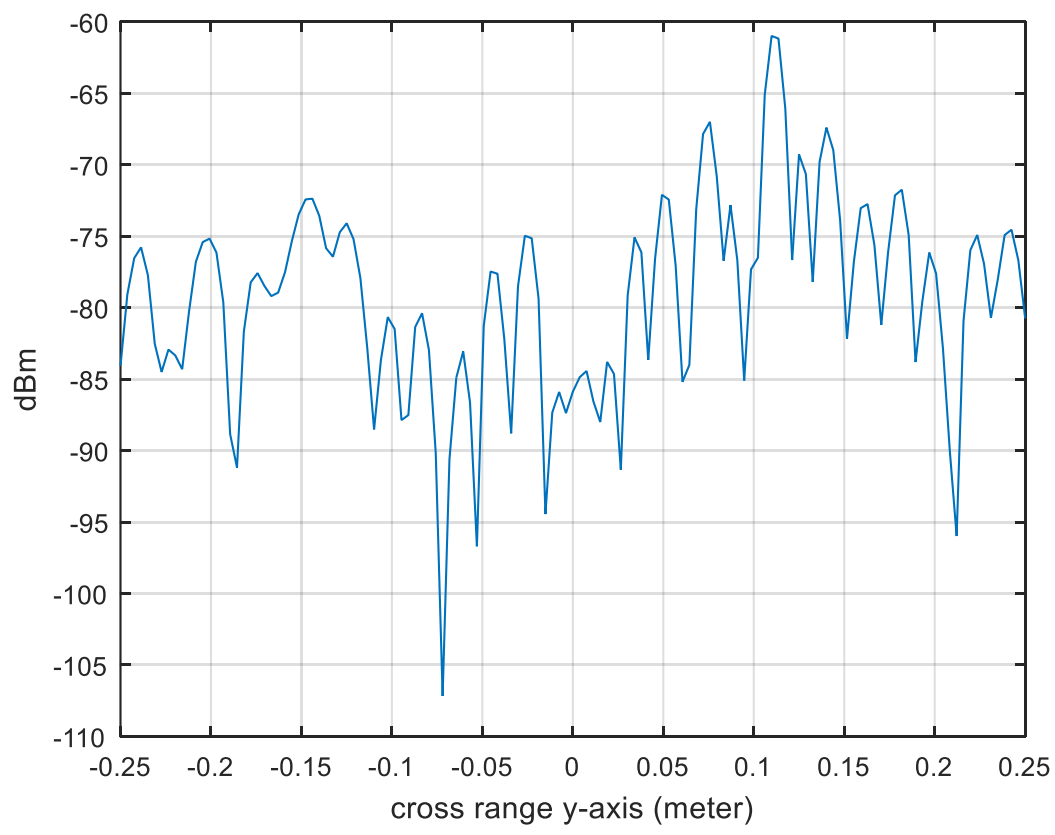

Figure 33: y-axis cross range of weak scatterer, showing the sidelobe suppressing weak scatterer signal after applying MRL algorithm. 


\subsection{Near-field CLEAN Algorithm Results}

The general idea of CLEAN algorithm is to subtract the estimate the dominant scatterer electromagnetics response from the image. The simulation was structured to form as image a regular and irregular complex targets shape. The simulations were performed with the same parameters for MRL algorithm results.

\subsubsection{Regular Complex Target Shape Geometry}

Figure 34 shows the weak scatterer as barely clear beside the ghost projection nearby for regular complex geometry shown in Figure 14. The power distribution in Figure 35 of the CLEAN image shows the weak scatterer power was degraded when the PSF of the dominant scatterer was removed from the received signal. A close look for the weak scatterer PSF power signal shown in Figure 36 and Figure 37 for cross section view at $x$-axis and $y$-axis respectively. 


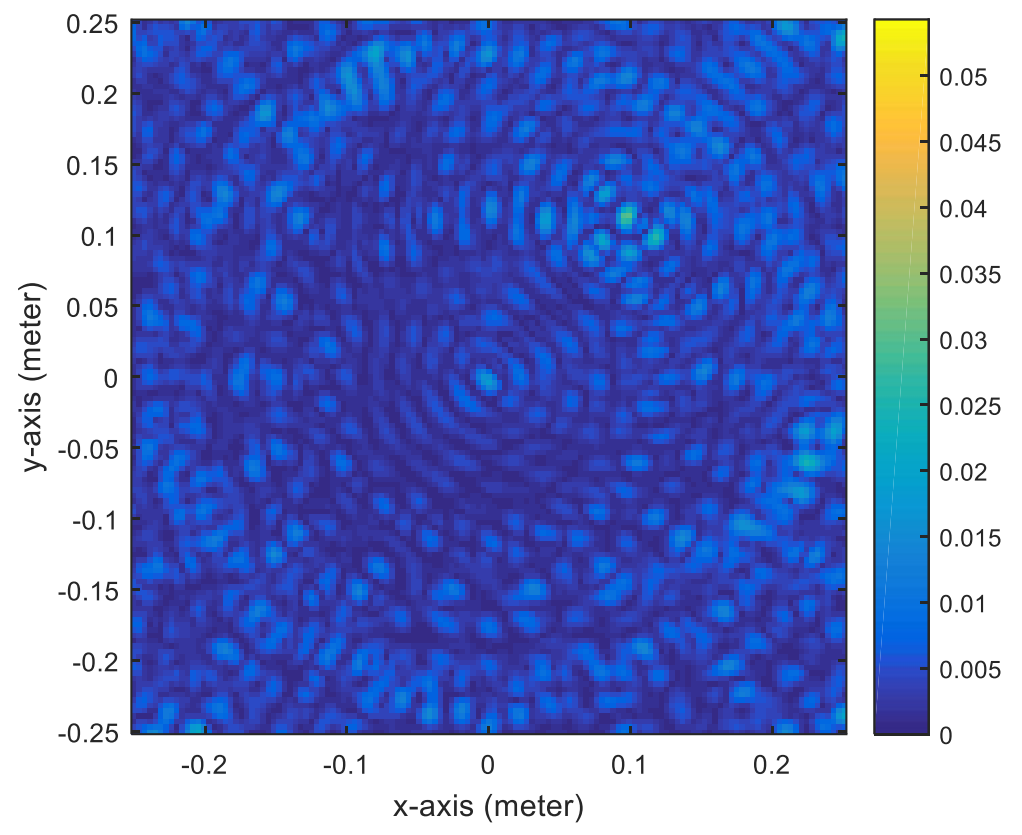

Figure 34: The results of applying CLEAN algorithm for regular complex shape geometry.

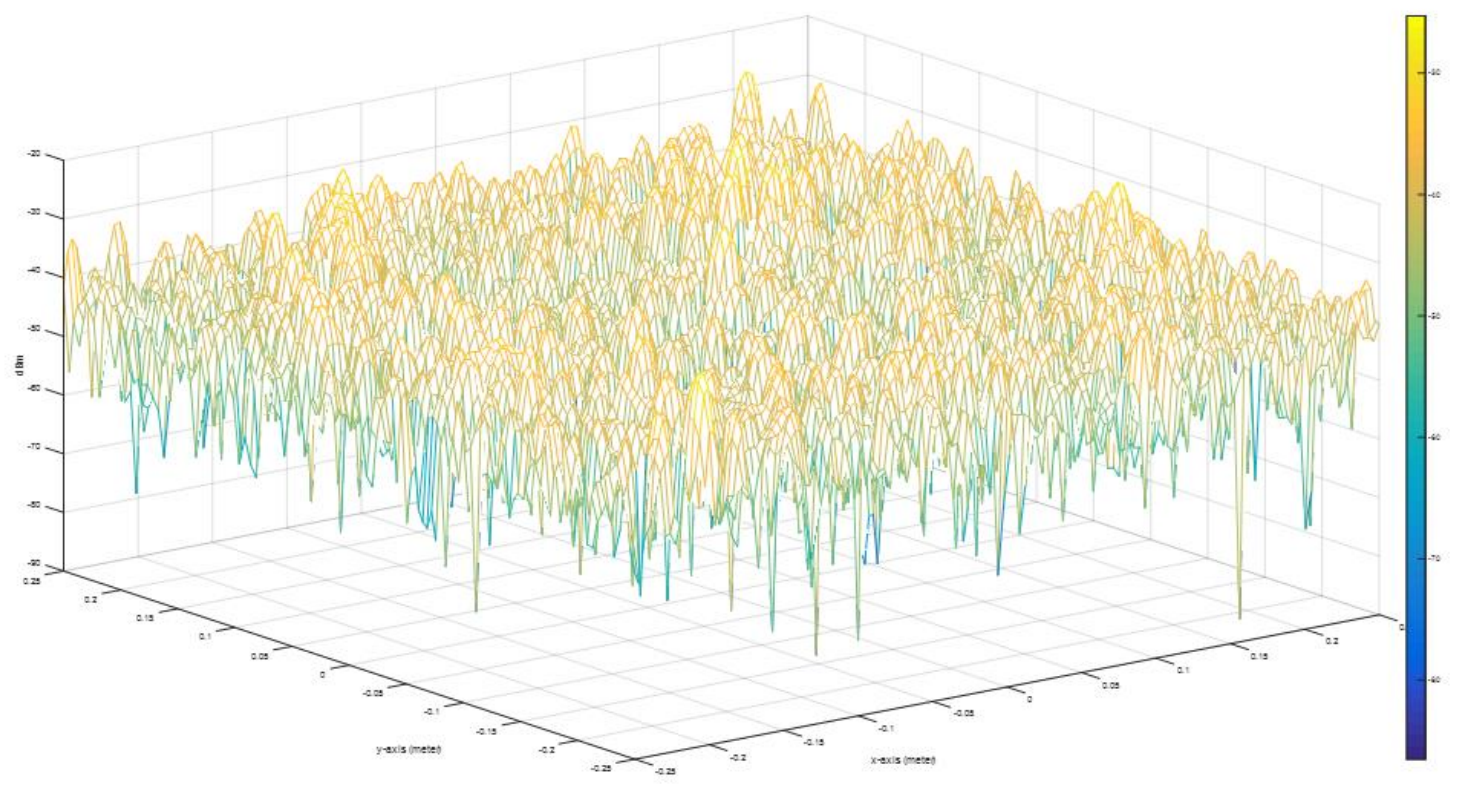

Figure 35: The power distribution of tomographic image after applying CLEAN algorithm for regular complex target shape geometry. 


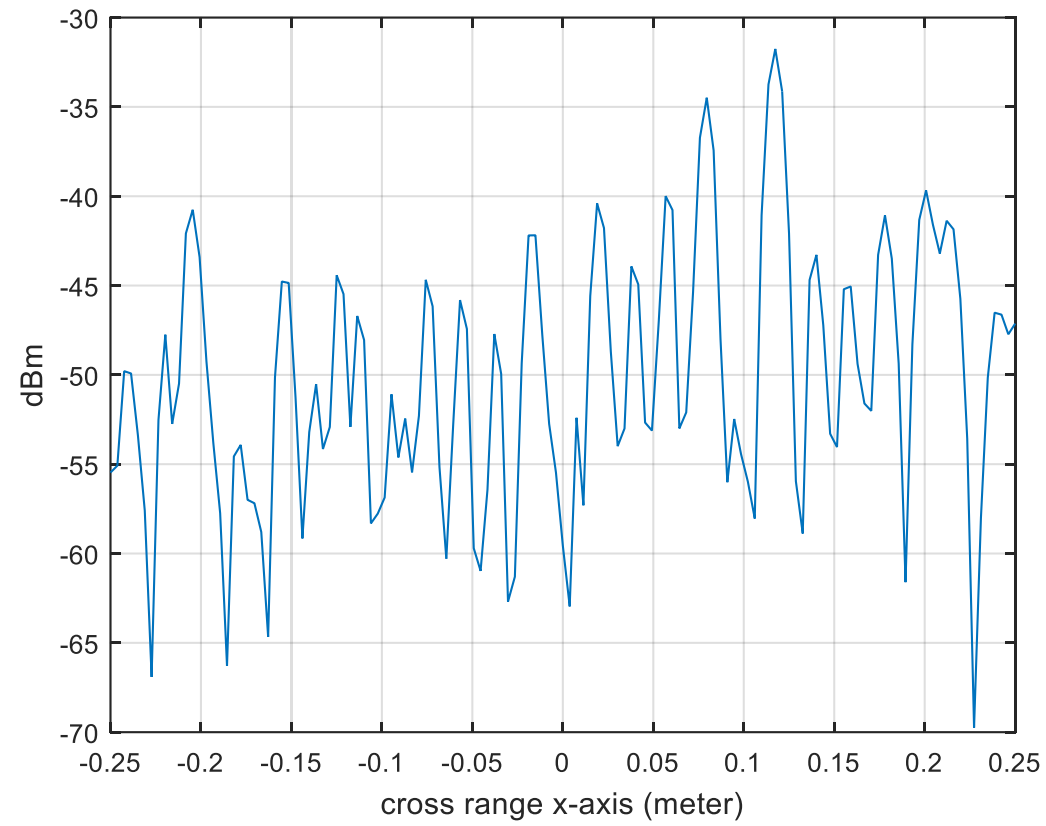

Figure 36: The sectional view of intensity of weak scatterer and sidelobe at $x$-axis after applying CLEAN algorithm for regular complex target shape geometry.

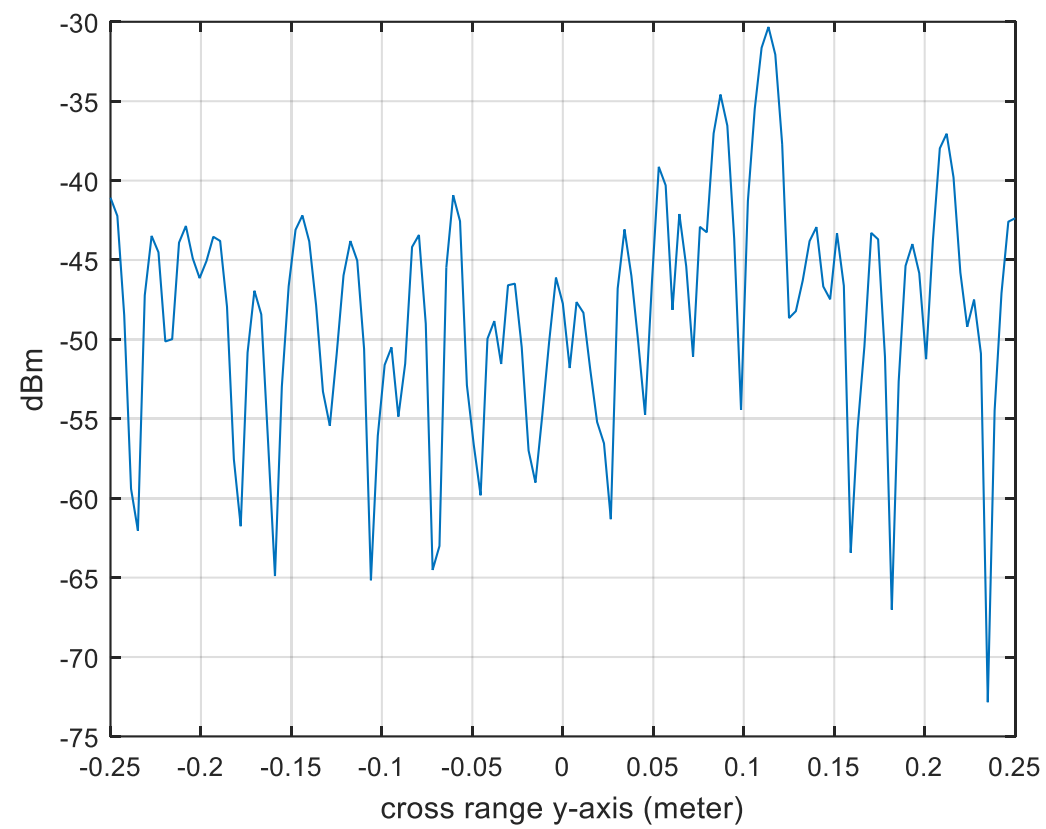

Figure 37: The sectional view of intensity of weak scatterer and sidelobe at y-axis after applying CLEAN algorithm for regular complex target shape geometry. 


\subsubsection{Irregular Geometry}

The irregular complex shape geometry in Figure 25 shows no improvement in the weak scatterer detection. The weak scatterer PSF was extracted with from the received signal as shown in Figure 38. The power distribution in Figure 39 is still high compared to the weak scatterer cross range view shown in Figure 40 and Figure 41.

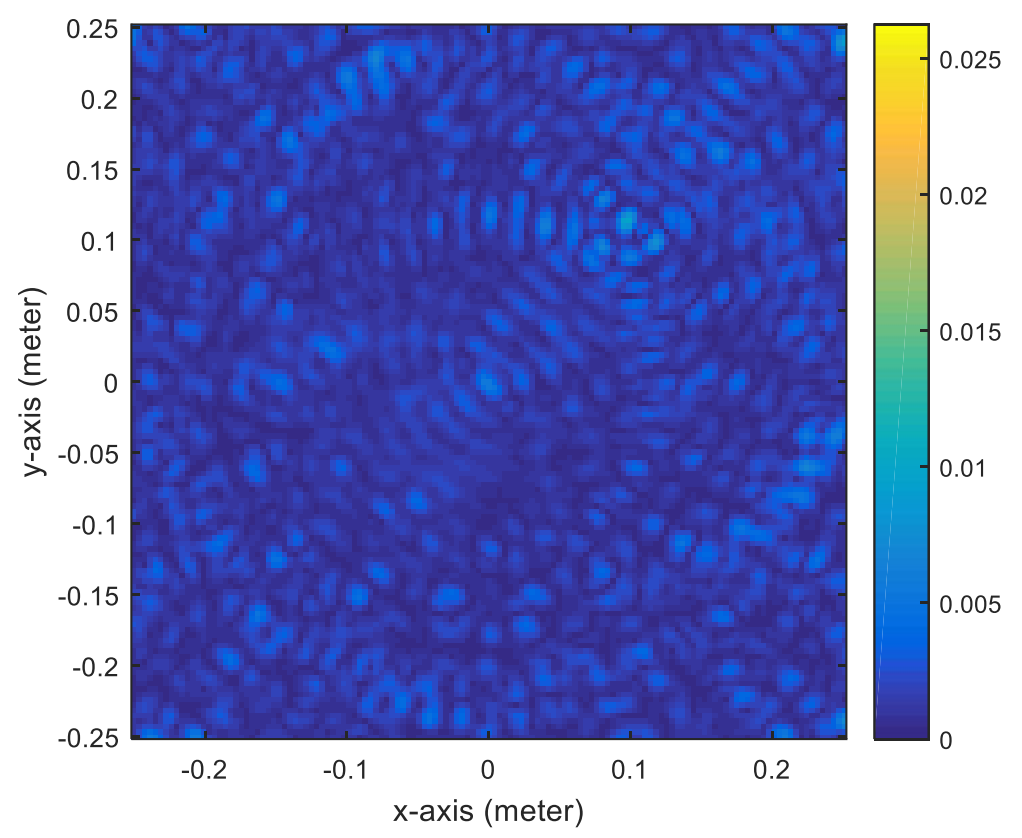

Figure 38: The results of applying CLEAN algorithm for irregular complex shape geometry 


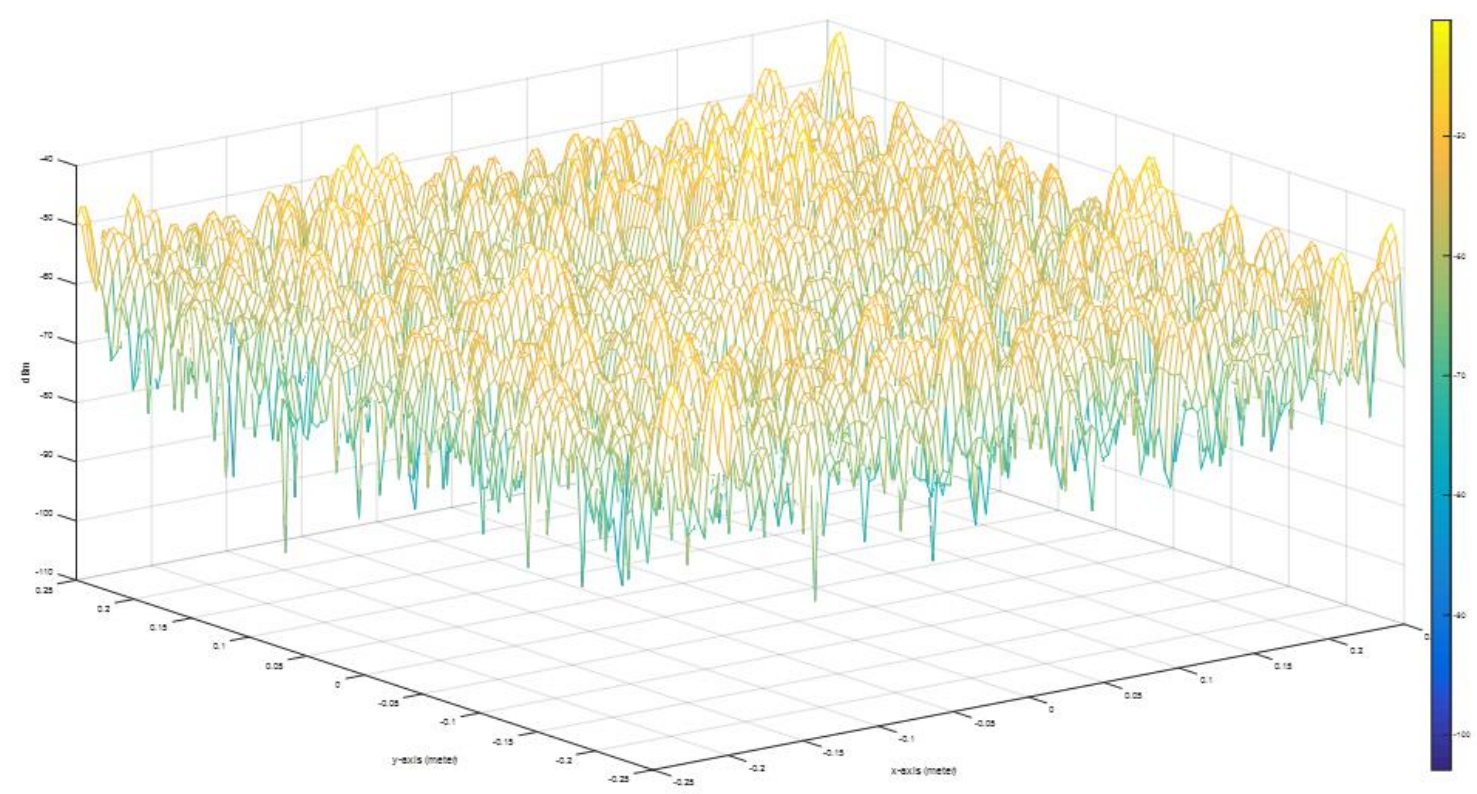

Figure 39: The power distribution of tomographic image after applying CLEAN algorithm for irregular complex target shape geometry.

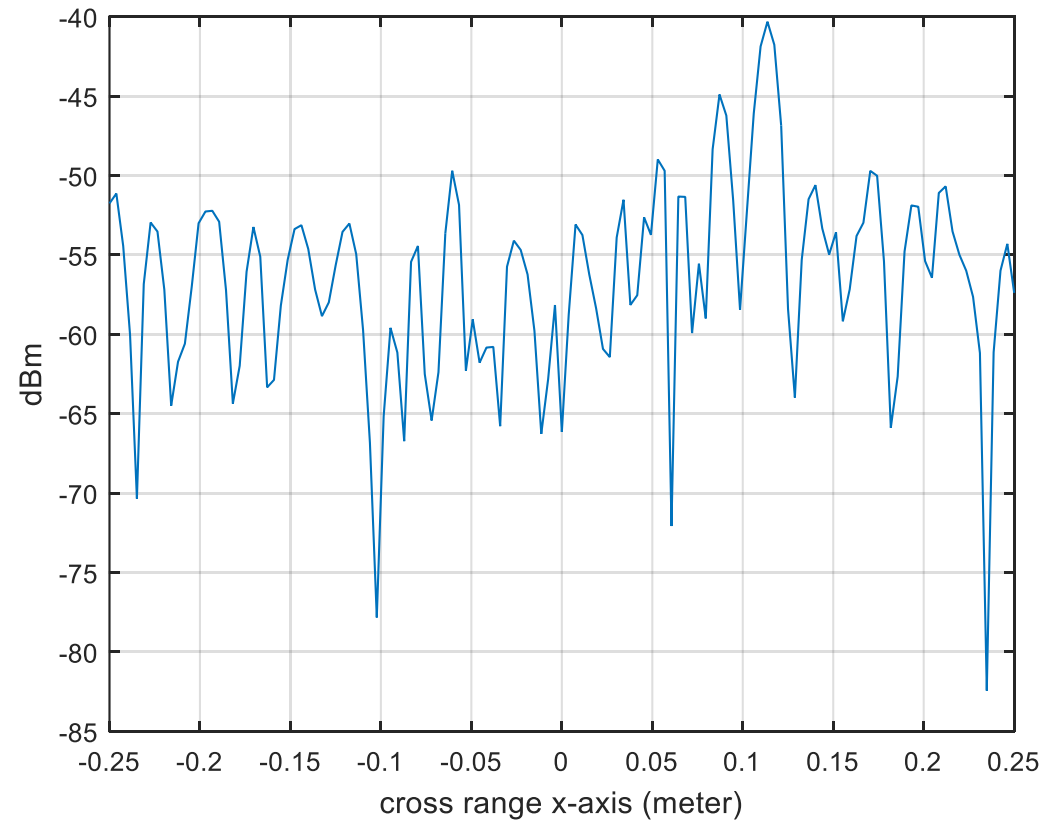

Figure 40: The sectional view of power density and sidelobe at $x$-axis after applying CLEAN algorithm for irregular complex target shape geometry. 


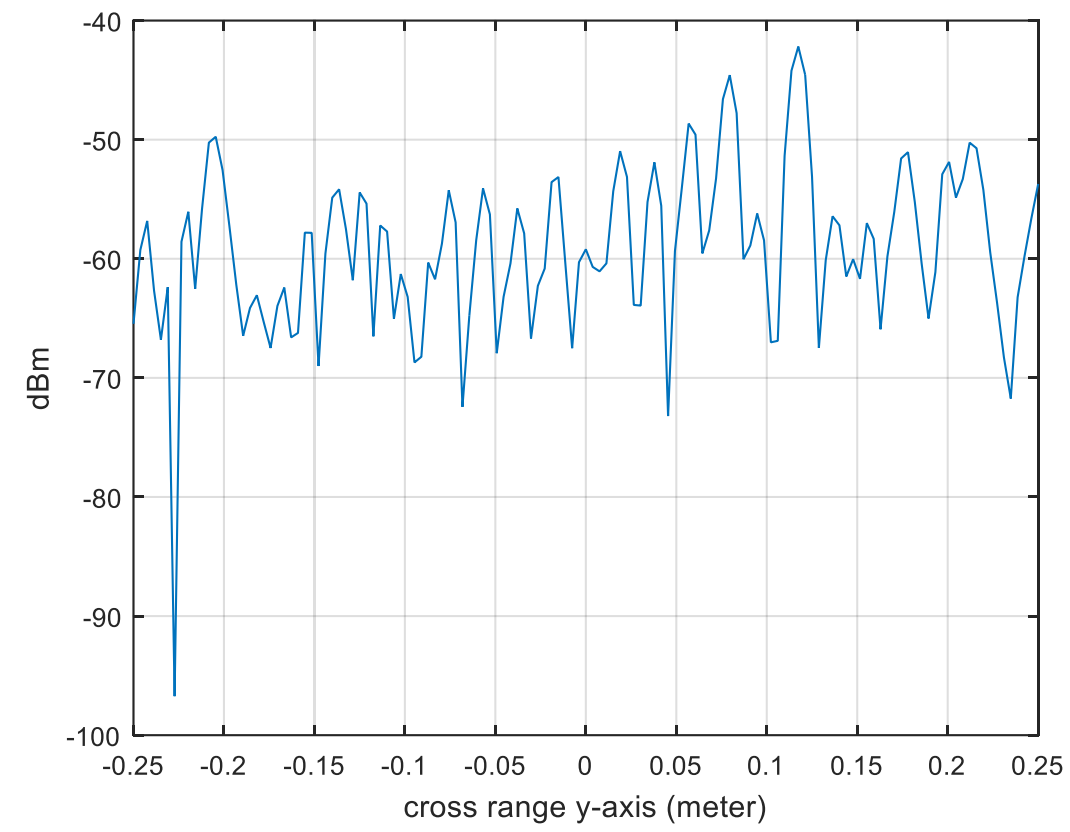

Figure 41: The sectional view of power density and sidelobe at $y$-axis after applying CLEAN algorithm for irregular complex target shape geometry.

\subsection{Irregular Geometry SNR Analysis}

The visibility of a weak scatterer is increased when we used MRL algorithm versus other approaches. To present the improvement in resolution, as mentioned in previous chapters, the resolution is improved via increased SNR. Here we selected an irregular geometry to analyze the SNR improvement obtained under the Born approximation, applying the CLEAN algorithm, and the MRL algorithm. The irregular geometry exhibits the highest noise (interference) as compared to other geometries. Table 1 presents the weak scatter signal, in both $\mathrm{y}$ and $\mathrm{x}$, since the PSF of the dominant scatterer changes in azimuth in near-field region. The weak scatterer is located at $0.1 \mathrm{~m}$ in $\mathrm{x}$ and $0.112 \mathrm{~m}$ in $\mathrm{y}$. 
Table 1: SNR analysis for irregular geometry.

\begin{tabular}{|c|c|c|c|c|c|c|}
\hline & \multicolumn{3}{|c|}{$\mathrm{x}$-axis-range } & \multicolumn{3}{|c|}{ y-axis-range } \\
\hline & $\begin{array}{c}\text { Weak } \\
\text { Scatterer } \\
\text { Intensity }\end{array}$ & $\begin{array}{l}\text { Range } \\
\text { Mean }\end{array}$ & SNR & $\begin{array}{c}\text { Weak } \\
\text { Scatterer } \\
\text { Intensity }\end{array}$ & $\begin{array}{l}\text { Range } \\
\text { Mean }\end{array}$ & SNR \\
\hline $\begin{array}{c}\text { Born } \\
\text { Approx. }\end{array}$ & $\begin{array}{c}-62.94 \\
\mathrm{dBm}\end{array}$ & $\begin{array}{c}-64.8445 \\
\mathrm{dBm}\end{array}$ & $\begin{array}{c}1.9045 \\
\mathrm{dBm}\end{array}$ & $\begin{array}{c}-50.42 \\
\mathrm{dBm}\end{array}$ & $\begin{array}{c}-62.4165 \\
\mathrm{dBm}\end{array}$ & $\begin{array}{c}11.9965 \\
\mathrm{dBm}\end{array}$ \\
\hline CLEAN & $\begin{array}{c}-60.98 \\
\mathrm{dBm}\end{array}$ & $\begin{array}{c}-51.4312 \\
\mathrm{dBm}\end{array}$ & $\begin{array}{c}-9.4588 \\
\mathrm{dBm}\end{array}$ & $\begin{array}{c}-35.56 \\
\mathrm{dBm}\end{array}$ & $\begin{array}{c}-50.5273 \\
\mathrm{dBm}\end{array}$ & $\begin{array}{c}14.9673 \\
\mathrm{dBm}\end{array}$ \\
\hline MRL & $\begin{array}{c}-61.17 \\
\mathrm{dBm}\end{array}$ & $\begin{array}{c}-79.3802 \\
\mathrm{dBm}\end{array}$ & $\begin{array}{c}18.2101 \\
\mathrm{dBm}\end{array}$ & $\begin{array}{c}-62.22 \\
\mathrm{dBm}\end{array}$ & $\begin{array}{c}-79.7887 \\
\mathrm{dBm}\end{array}$ & $\begin{array}{c}17.5673 \\
\mathrm{dBm}\end{array}$ \\
\hline
\end{tabular}

The SNR obtained via the MRL algorithm indicates a stable value of the intensity over the mean interference in both $\mathrm{x}$ and $\mathrm{y}$. Moreover, the sidelobe level is reduced to maximize the SNR. The weak scatterer intensity under the Born approximation and CLEAN algorithm (in $\mathrm{x}$ ) had a smaller SNR value than in $\mathrm{y}$ because the sidelobe in $\mathrm{x}$ is increased. Furthermore, the radiation pattern of the domain scatterer is masking the weak scatterer in y. Comparing all three algorithms, we can say the MRL algorithm sufficiently increases the SNR in near-field region. 


\section{CHAPTER 7}

\section{EXPERIMENTAL SETUP}

The experimental setup for semi-anechoic chamber introduced here was held on a free space environment with dimensions $3.8 \times 3.87 \times 2.14 m$ shown in Figure 42 . The absorber materials are used to reduce the electromagnetic reflection from walls and ceiling. Designing an automated Radar tomographic system in Mumma Radar Laboratory has required many steps to overcome numerous difficulties. The First Step to obtain an accurate signal is to calibrate the position of the transmitters and the receivers in our systems. Motoman DX100 Robots and turntable are designed to move in accurate micrometer scale are shown in Figure 1. Errors in spatial frequency happen due to the robot, cables and antennas group delay in the measuring system. We used Agilent (N5221A) eight ports costume made vector network analyzer (VNA) to measure the scattering field through Sparameters. The different length coaxial cables are used to connect VNA with the Horn antenna. Finding the most effective factor that causing an error was needed to determine which calibration technique fit our Mumma Radar laboratory environment. A bi-static measurement was shown an error of the location of a metal target plate. A metal plates were used at different dimensions, the big plate is $26 \mathrm{~cm}$ by $22 \mathrm{~cm}$, the medium plate is 11 by $11 \mathrm{~cm}$, and the small plate is 5 by $5 \mathrm{~cm}$ (Figure 46, Figure 47 and Figure 48). The RCS 
has different values for each dimension which represent the scattered electric field at the target. The measurement for these targets plates were shown in Figure 45 was done from 2 to $18 \mathrm{GHz}$. Background subtraction was made to clear the scene from permanent clutter; the background subtraction done be measuring the electric scattered field of the scene when the target is absent then subtracted from the scattered electric field when target present. The error in these plate measurements were nonlinear due to near-field measurement, high directivity of the antennas, and the mutual coupling. Knowing these factors provide us a clear vision of what we can expect in our measurement in semi-anechoic chamber. The semi-anechoic chamber is predicting a real world environment. Most anechoic chamber work in reflection free zone or work in millimeter wave zone where the reflection can be neglecting. The large metal robotic arms has no reflection effect on the accuracy of the measurement. As we predict the measurement scene we applied a calibration procedure well known in the literature. 


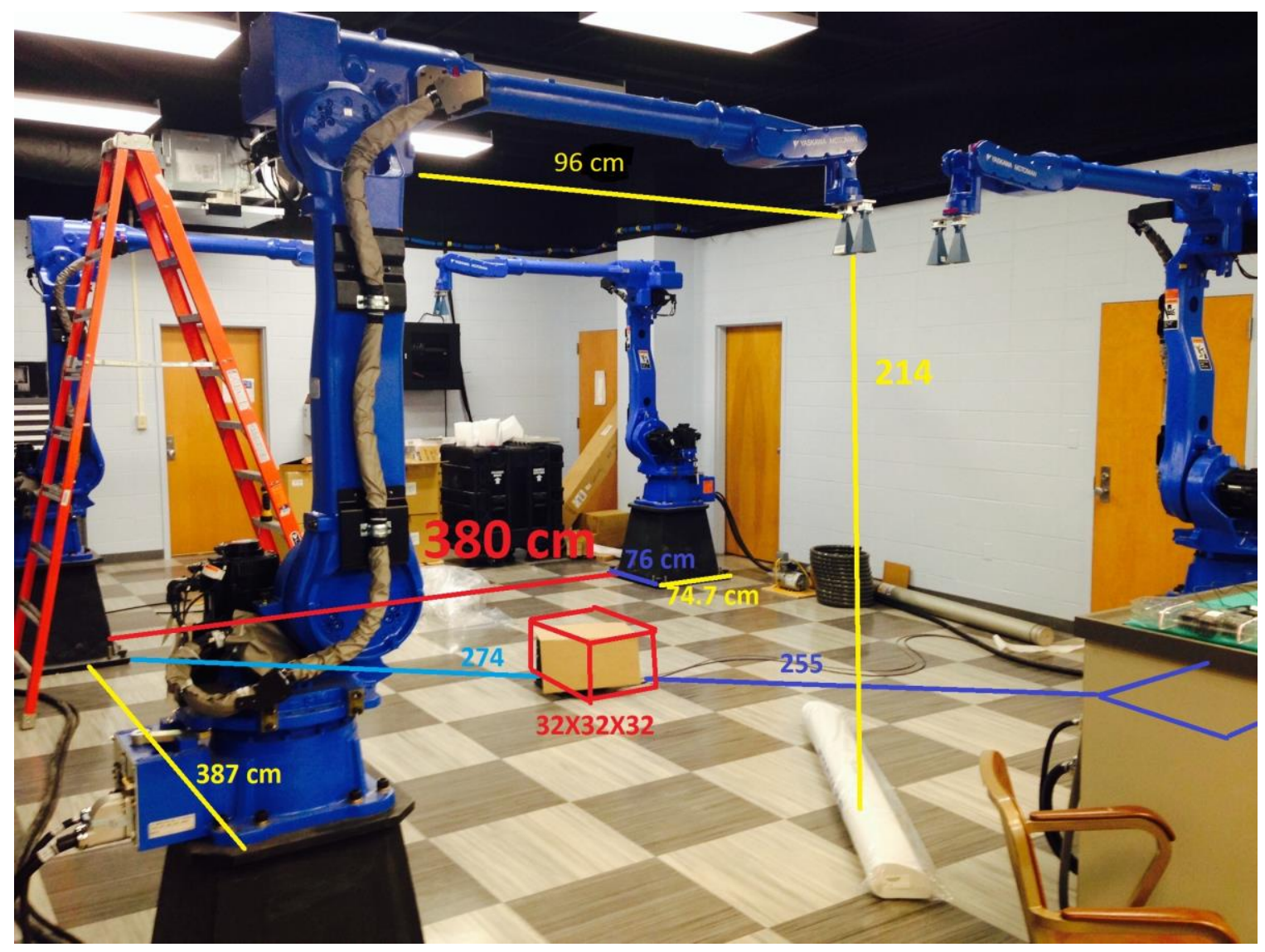

Figure 42: The anechoic chamber dimensions

\subsection{Sphere Calibration}

The following steps do the calibration procedure. First, a calibration target for calibration purposes was selected and used to obtain a measurement of the scattered electric field. For this study, a $15 \mathrm{~cm}$ metal sphere was selected as shown in Figure 51. The sphere was hung to one of the robotic arms. Eight horn antennas with fixed locations were used to measure the scattered electric field as presented in Figure 51. The Horn array antennas was designed as a quarter circular trajectory with radius of $1.5 \mathrm{~m}$. A single Horn antenna mount is directed at the center of the circle which is also the center of the robotic turntable. The robotic turntable can rotate 360 degrees with variable speed with accuracy of 1 micrometer.

The electric field response of the metallic sphere $S_{21}^{\text {Sphere }}$ was collected as the S-parameter. 
The next step was to calculate the response of a sphere with the same dimension and location analytically. FEKO was used to simulate the electric field response using the method of moment which gives the exact response in free space when noise absent. FEKO model of the sphere is shown in Figure 49. The electric field response of FEKO simulation at frequency domain is shown in Figure 50.The reasons of this calibration procedure are to convert the S-parameter data obtained from VNA into Electric field $E$ values to be used in the inversion algorithms. The second reason is to eliminate any error during the measurement.

Finally, Electric scattered field of the measured sphere was giving by

$$
E_{\text {Calib }}^{\text {Scat }}=\frac{E_{F E K O}^{\text {Sphere }}}{S_{21}^{\text {Sphere }}} S_{21}^{\text {UnknownTarget }}
$$

Where $E_{\mathrm{FEKO}}^{\text {Spher }}$ denoted the theoretical scattered field of a sphere calculated via FEKO.

$S_{21}^{\text {Sphere }}$ is the actual scattered field measured by VNA as S-parameter. $S_{21}^{\text {UnknownTarget }}$ is unknown target to be measured.

This calibration method will remove errors such cable losses, phase shifts, and mismatches at connectors. The unknown target in the equation preferred to be other objects but not a sphere. Using a sphere for calibration and measuring will provide deceptive impression about error estimation as shown in Figure 52.

\subsection{Positioning System}

Carefully deploying the transmitters and receivers in exact positions will improve the imaging process by predicting the radiation pattern of the antenna and its electric field response at each pixel position. Some problems happened during the operation and 
calibration of the robotics arms. One of the problems was that the robot is programmed to find the shortest path between stopping points. The stopping points strategically placed to halt the robotics arms and turntable to take a snapshot of the electric field using VNA. The shortest path can be in different directions and heights. Care was taken to ensure that the arms were not moving longer than the cable or attempt to turn in an unexpected direction. Final placement of the measured target results from the robot movement. However, there is no interaction or communication between the robotics arms. Therefore, care must be exercised to avoid collisions between the robotic arms. This could be hazardous especially knowing that a single robotic arm weighs nearly $300 \mathrm{~kg}$ each. The solution to avoid crashes was to program each robot separately using MATLAB. A single robot will then move in the desired way to stop and scan a target. After we made sure the robot motion was safe, we operated all robotic arms to test the motion, while they are scanning the target. The previous process with targets, cable, and measurement equipment were absent for safety. As a further test, Motoman offers a useful simulation program to simulate movement of the robotics arms with the MATLAB code.

Another problem was the look angle of the antenna during the robot movement. The look angle of the antenna is the orientation of the electromagnetic polarization field during the measurement. As the robotic arms move the antenna rotates to the desired look angle to the center of the measurement domain. Controlling the direction of the antenna will control both the directivity and radiation pattern of the antenna. The solution of this problem is to calibrate the robotic arms so that the mount tool is sufficiently long to reach the measurement domain center. In this case, the robotic arm will try to keep the antennas look angle at the center point. For corner reflector measurements, this method was applied 
to trick the robotics arms keeping the phase center of the antenna in the desired direction as shown in Figure 43. One issue with the corner reflector measurement is that the directivity of the antennas can be summed and sent back to the antennas again. This results in the antennas receiving too high of a value of the scattered electric field with high RCS. This affects the phase angle of the received signal. To avoid this issue, the look angle of the antenna was set to avoid the high directive main beam from the antenna and circularly scan the corner reflector. The corner reflector was used for calibration purposes. 


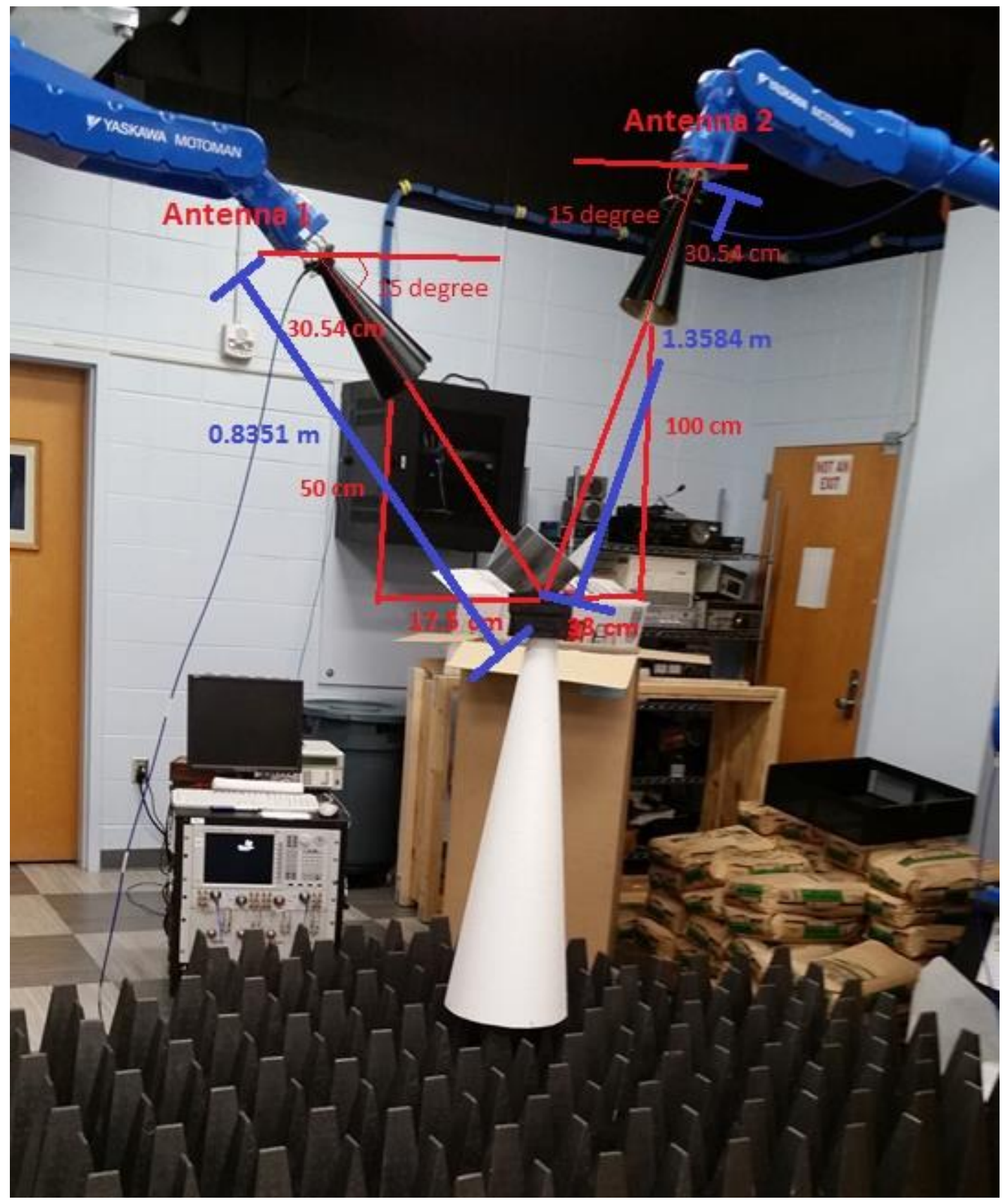

Figure 43: Two antenna looking at the center of corner reflector in calibration process. 


\section{D Printed}

Test and

Calibration

Targets

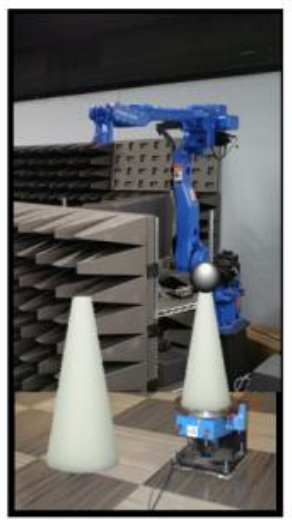

Rotating

Positioners and Target Pedestals

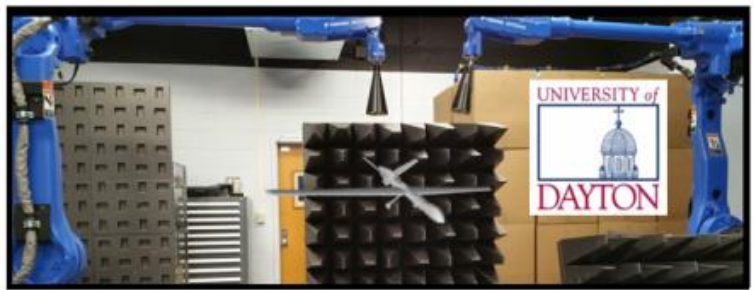

Calibrated

UWB

Polarized

Antennas
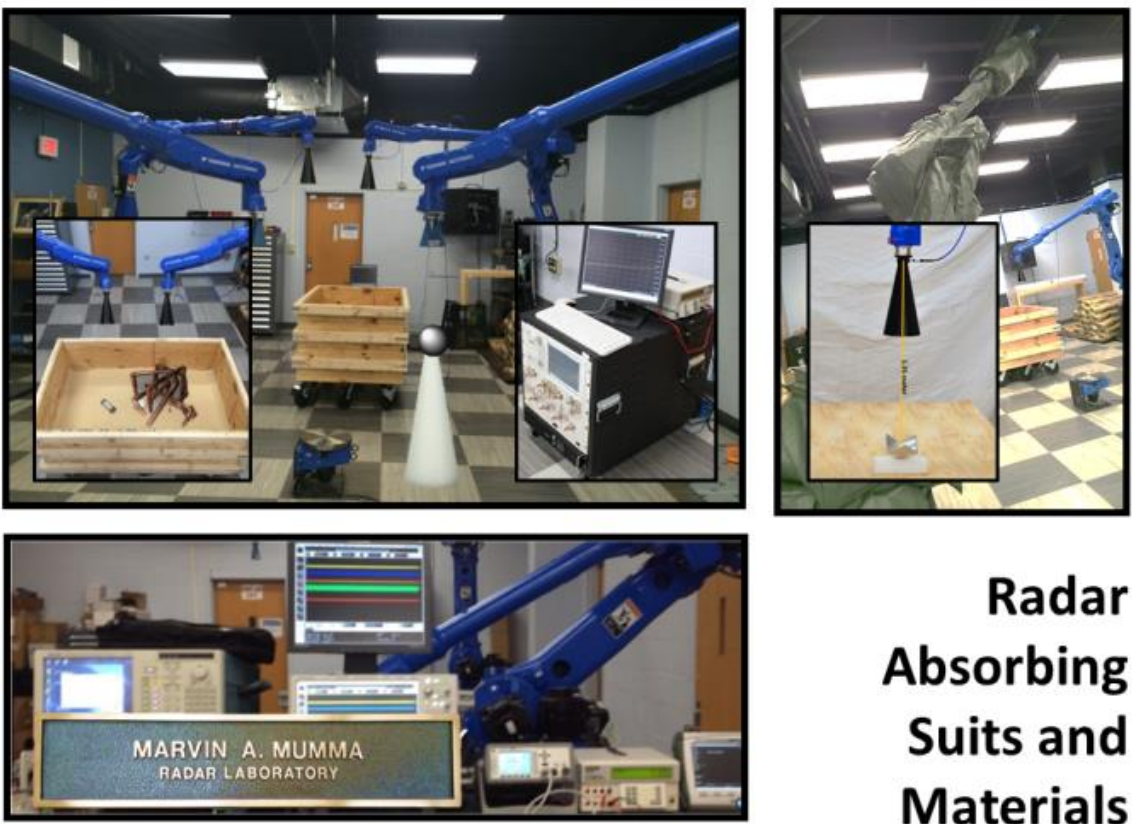

Radar Absorbing Suits and Materials

Figure 44: Four robotic arms controlled by DX100 in Mumma lab. 
Big Plate located at 31.5 and 35.5 from the antenna, The actual locations are 1.6002 and $1.8034 \mathrm{~m}$
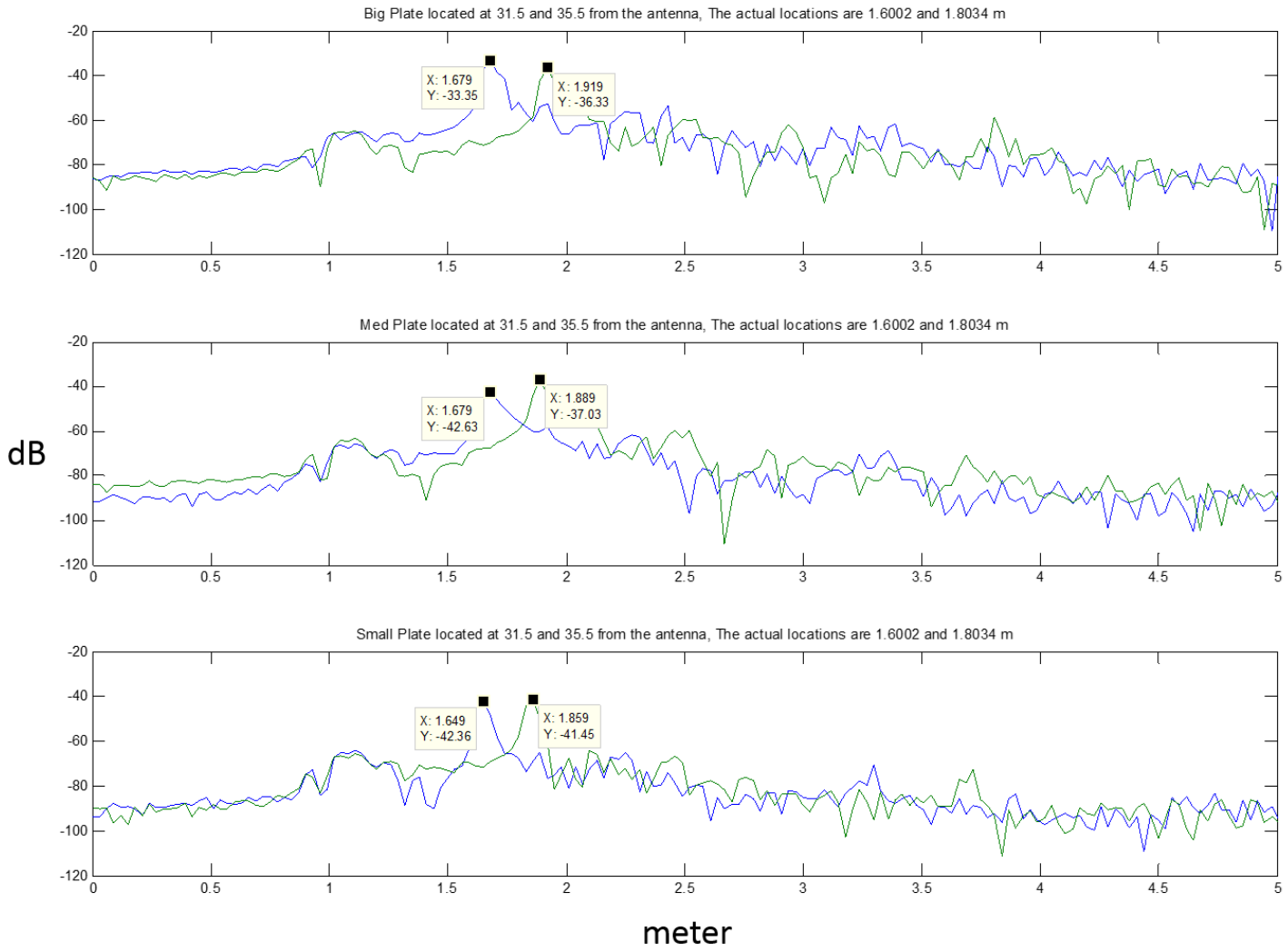

Figure 45: The results of scanning the targets in different locations
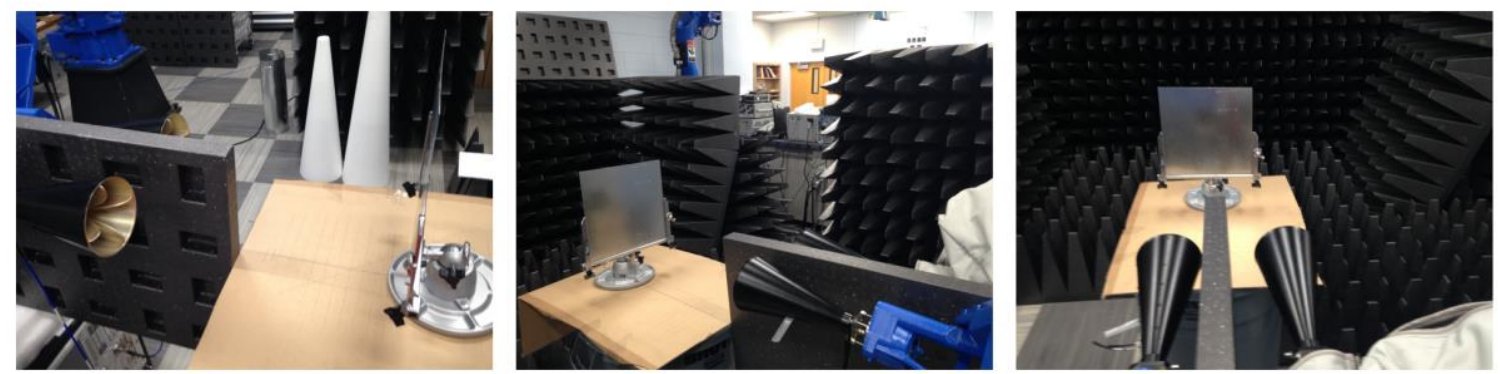

Figure 46: The big plate in the experiment set at three different locations. 


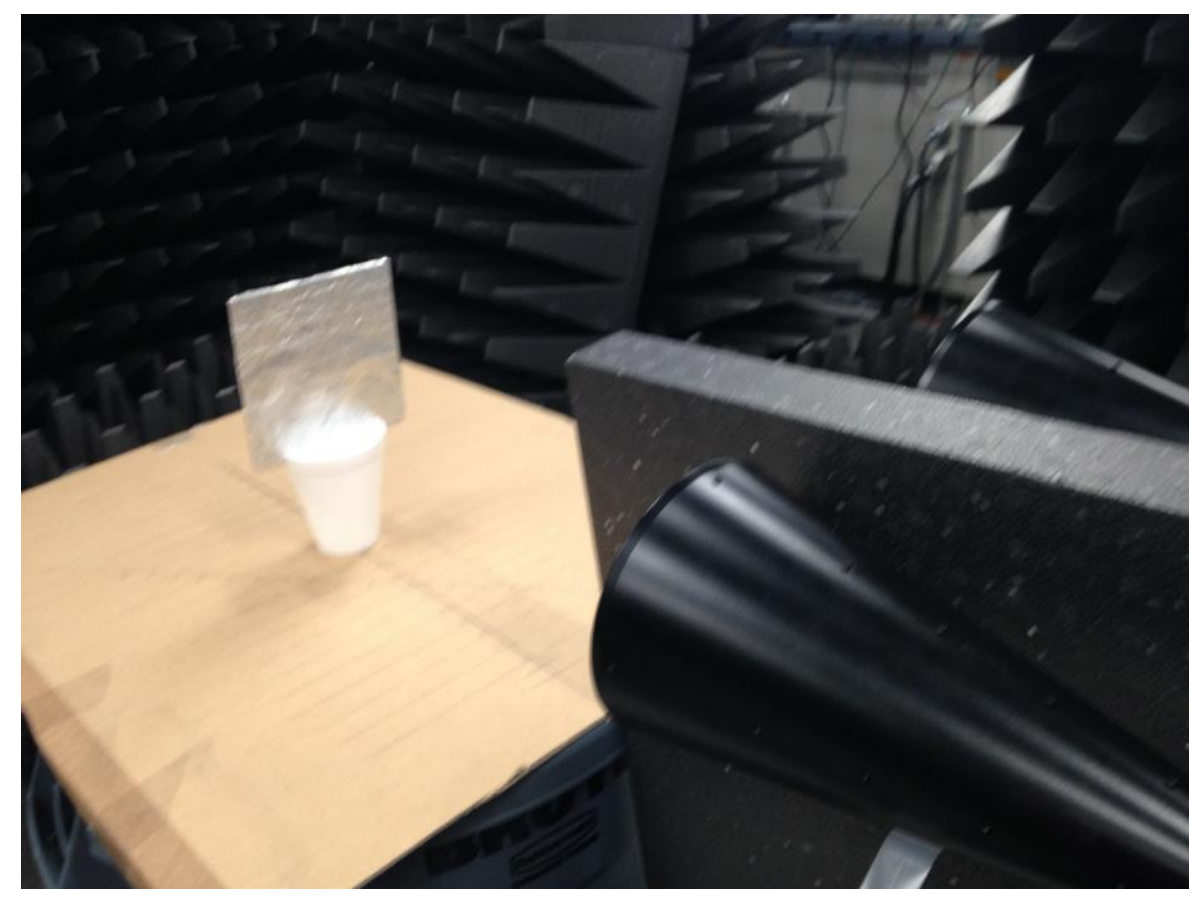

Figure 47: Medium plate in the experiment set.

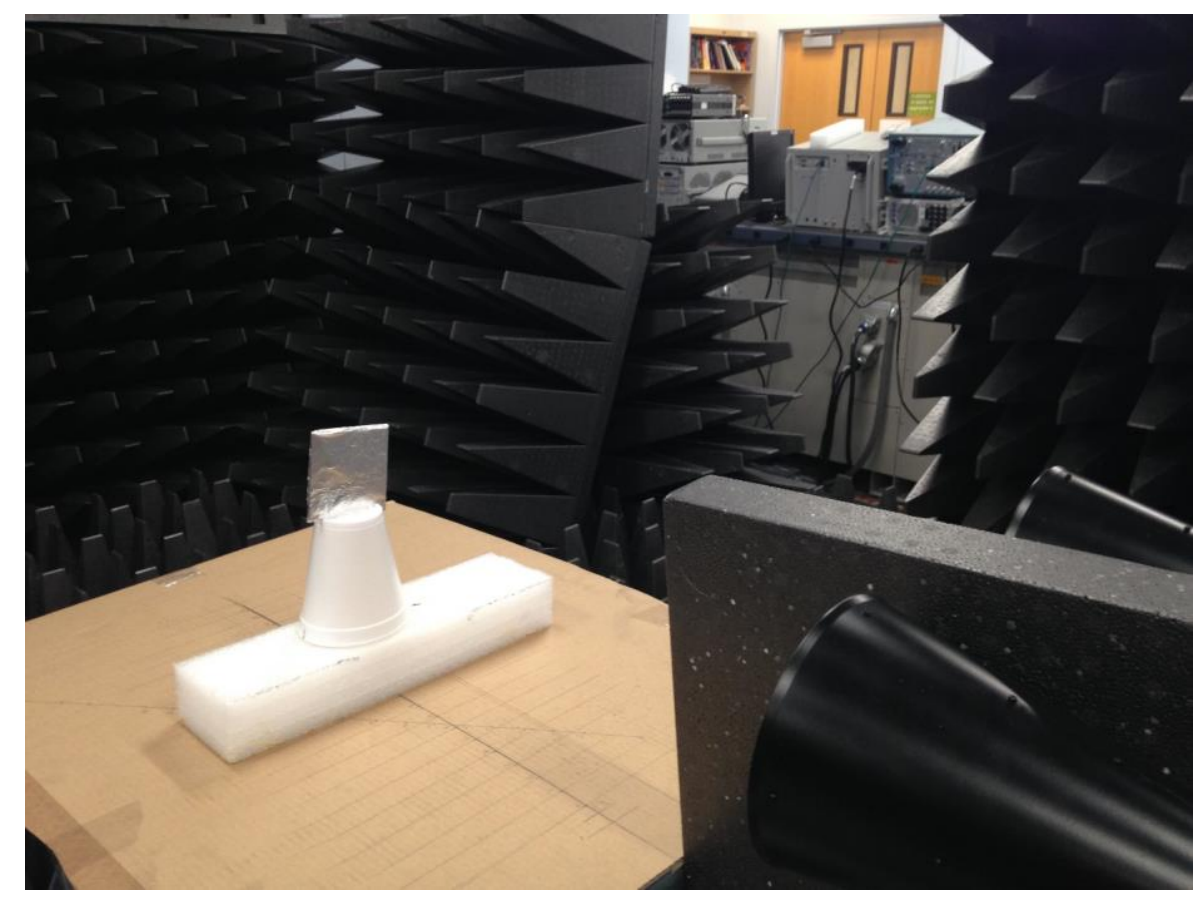

Figure 48: Small plate in the experiment set. 


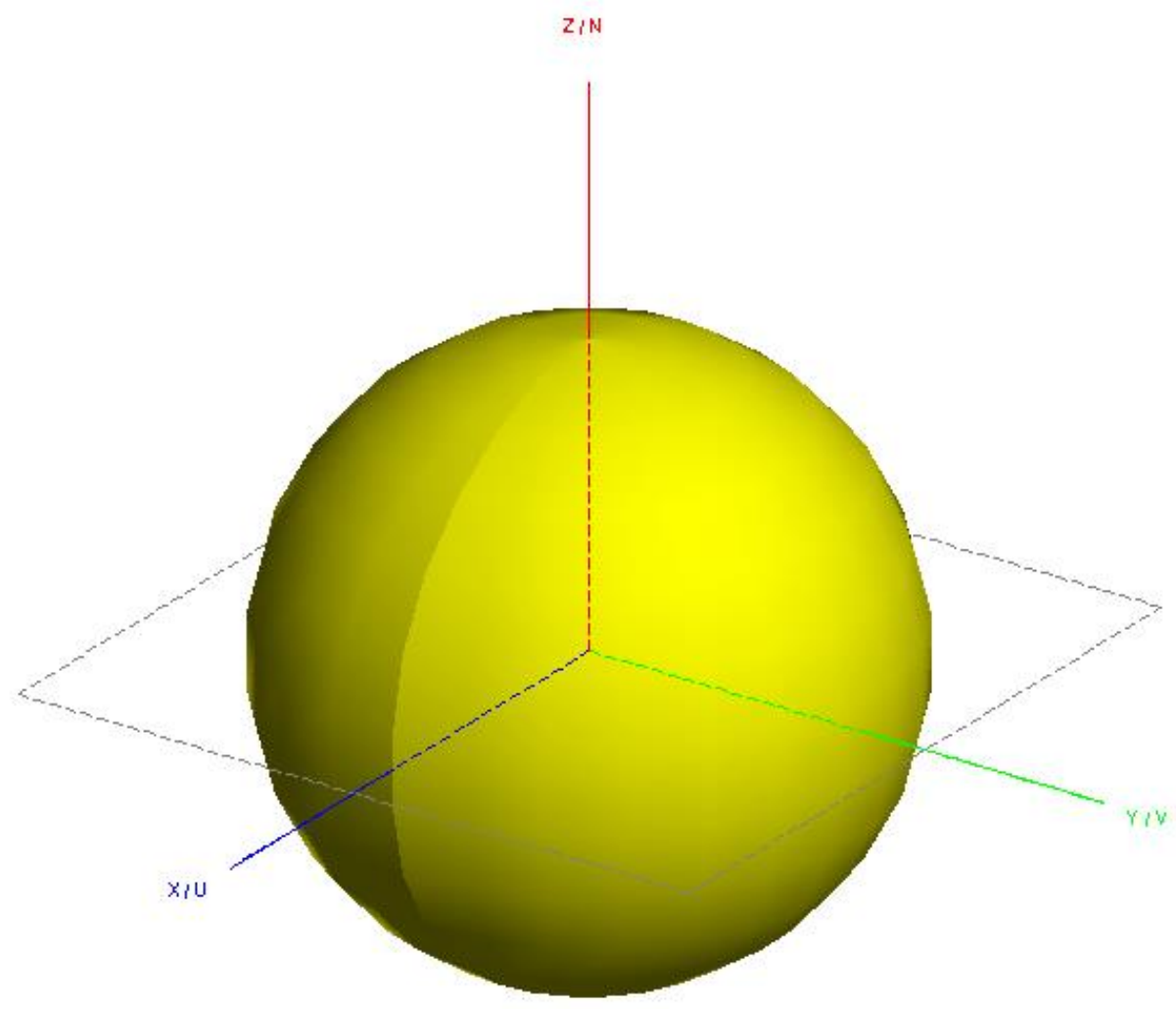

Figure 49: Simulating a sphere using FEKO tools.

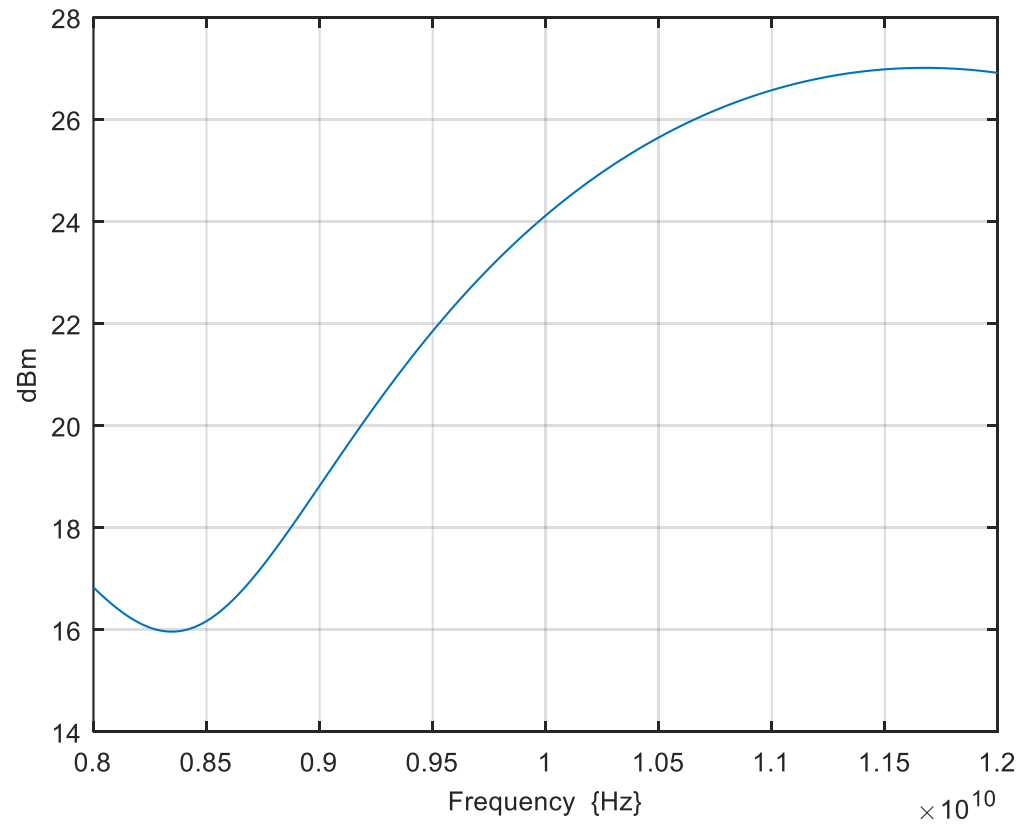

Figure 50: The scattering electric field of sphere versus frequency. 


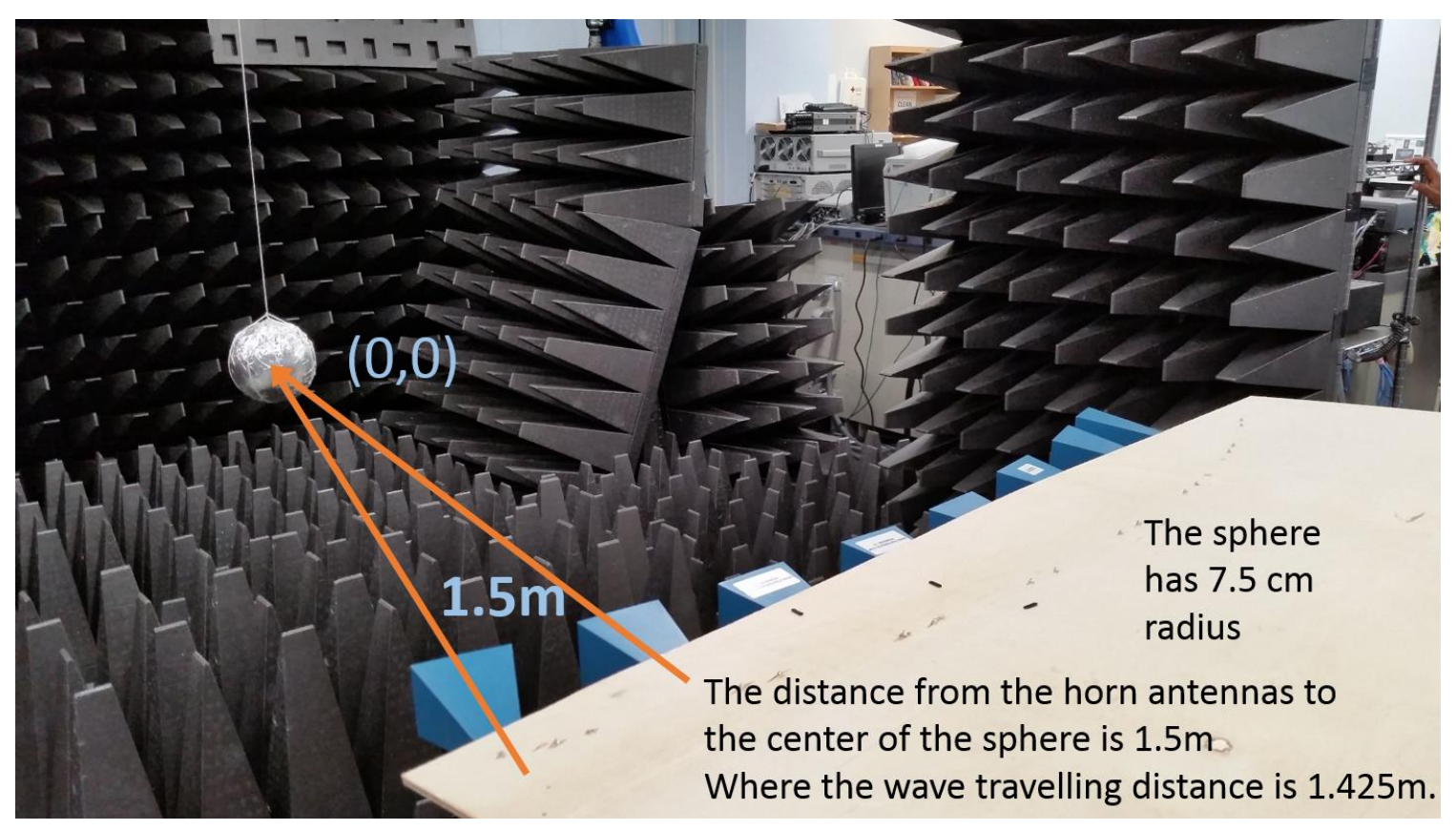

Figure 51: Sphere calibration measurement.

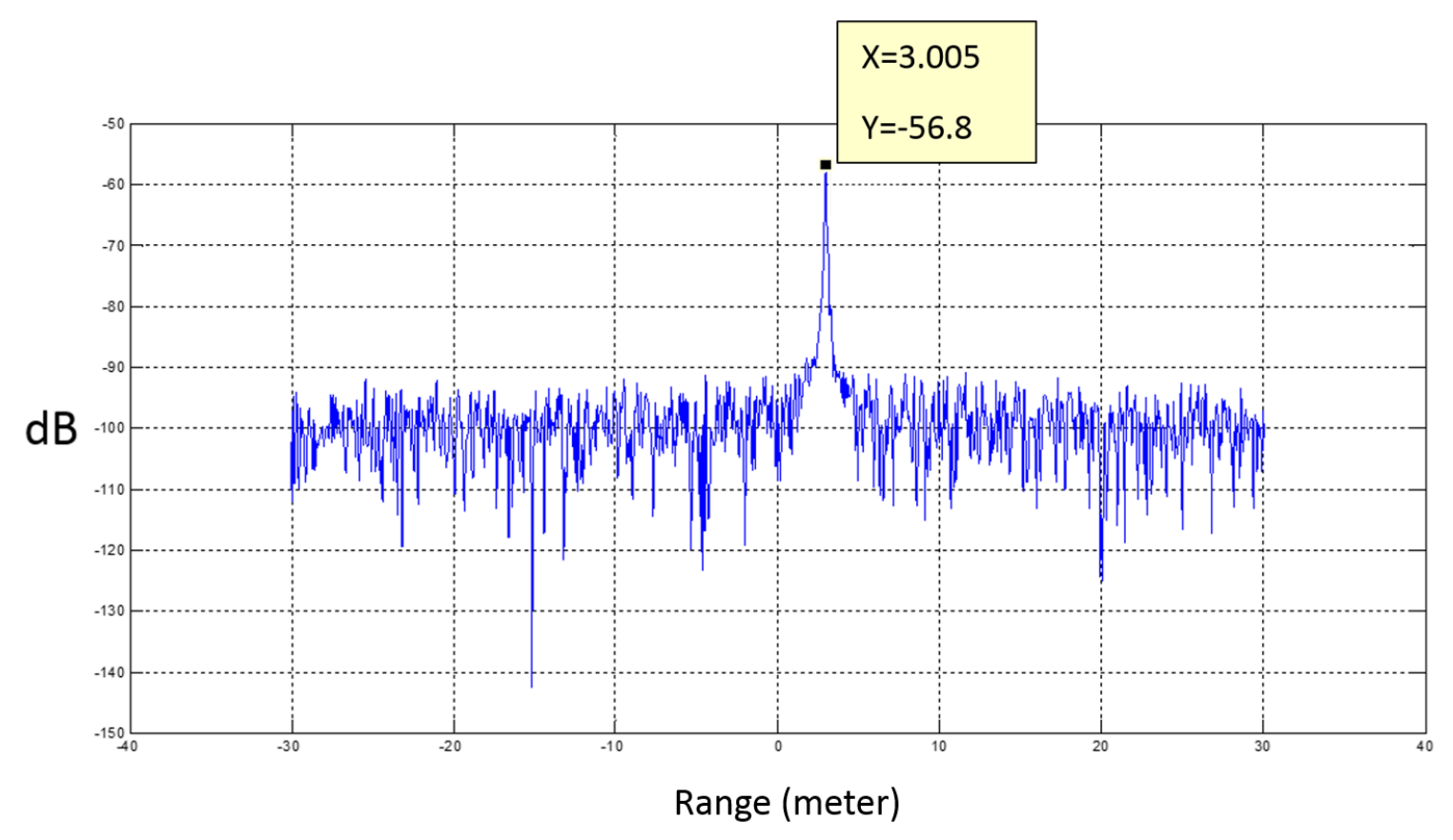

Figure 52: The response of the sphere with $2.5 \mathrm{~mm}$ error. 


\subsection{Single Cylinder Measurement}

The array of eight Horn antennas was fixed at 1.5 from the center. At the center the turn table was placed. Above the turn table a single cylinder was mounted. The distance between each single Horn antenna and the cylinder was fixed at $1.5 \mathrm{~m}$. The cylinder height was measured at $20.32 \mathrm{~cm}$ with a $2 \mathrm{~cm}$ radius. The cylinder rotates 360 degrees at variable speed. Initial measurement of the cylinder was made without calibrating the received signal as shown in Figure 54 The travelling distance between any two antennas was fixed to be $3 \mathrm{~m}$. The measurement showed the cylinder to be $14.54 \mathrm{~m}$ away. The source of the shift error are from the mismatches, cable lengths and cable losses. The first peak appear was observed at $12.14 \mathrm{~m}$ due to mutual coupling between the antennas. Likely some of the signal went to the other antenna before heading to the cylinder. The background measurement as shown in the middle plot shows that the response of the turn table is high. However, this can be considered as clutter The last plot at Figure 54 shows the cylinder at 14.52m with $-68.83 \mathrm{~dB}$ power. Next, we applied the sphere calibration equation to obtain calibrated measurement. The cylinder appears at $3.073 \mathrm{~m}$ with $3.65 \mathrm{~mm}$ error. The background subtraction presents the target at the same location with $-47.05 \mathrm{~dB}$ power. 


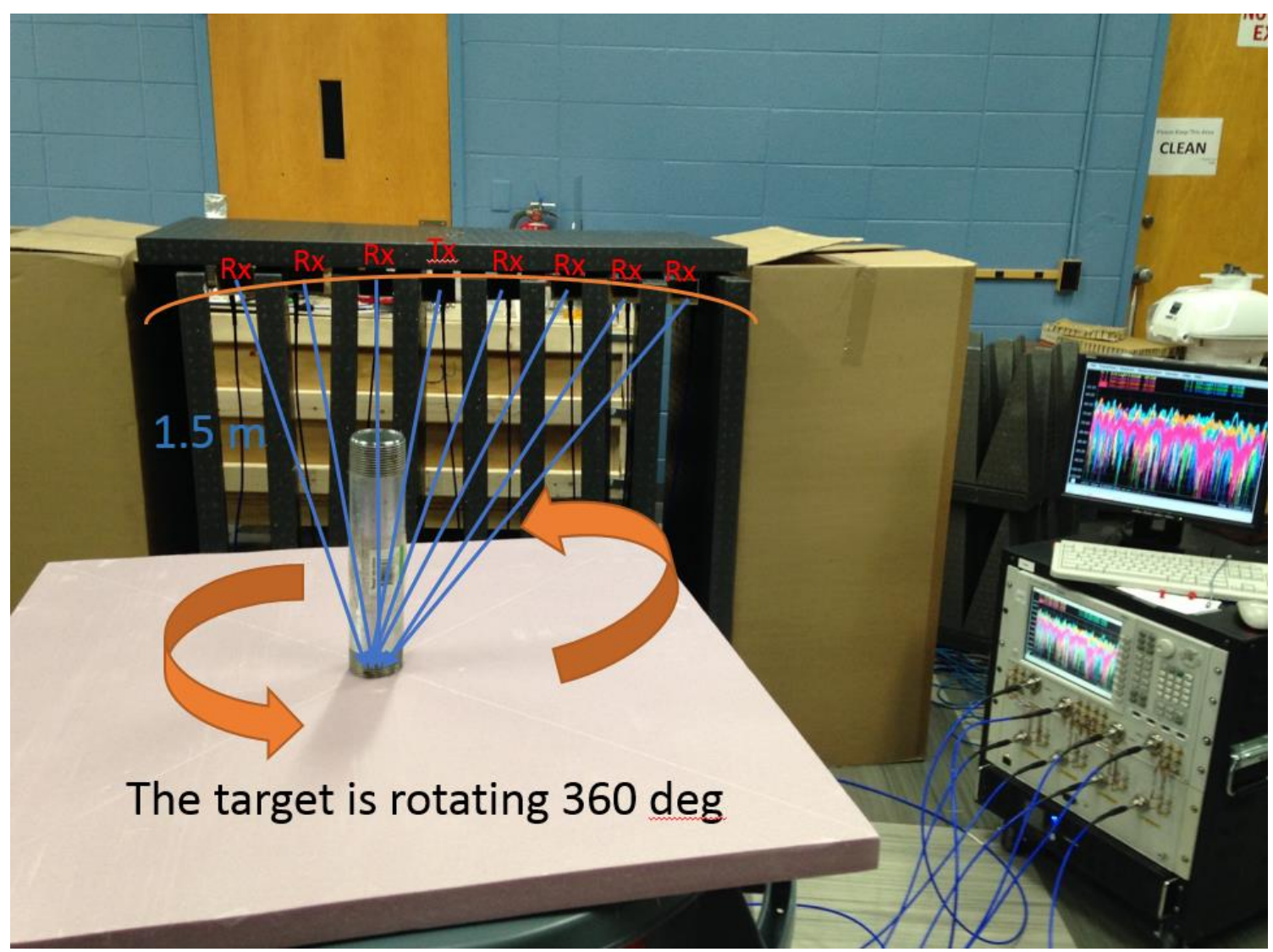

Figure 53: Measuring the scattering field for 8 inches cylinder. 

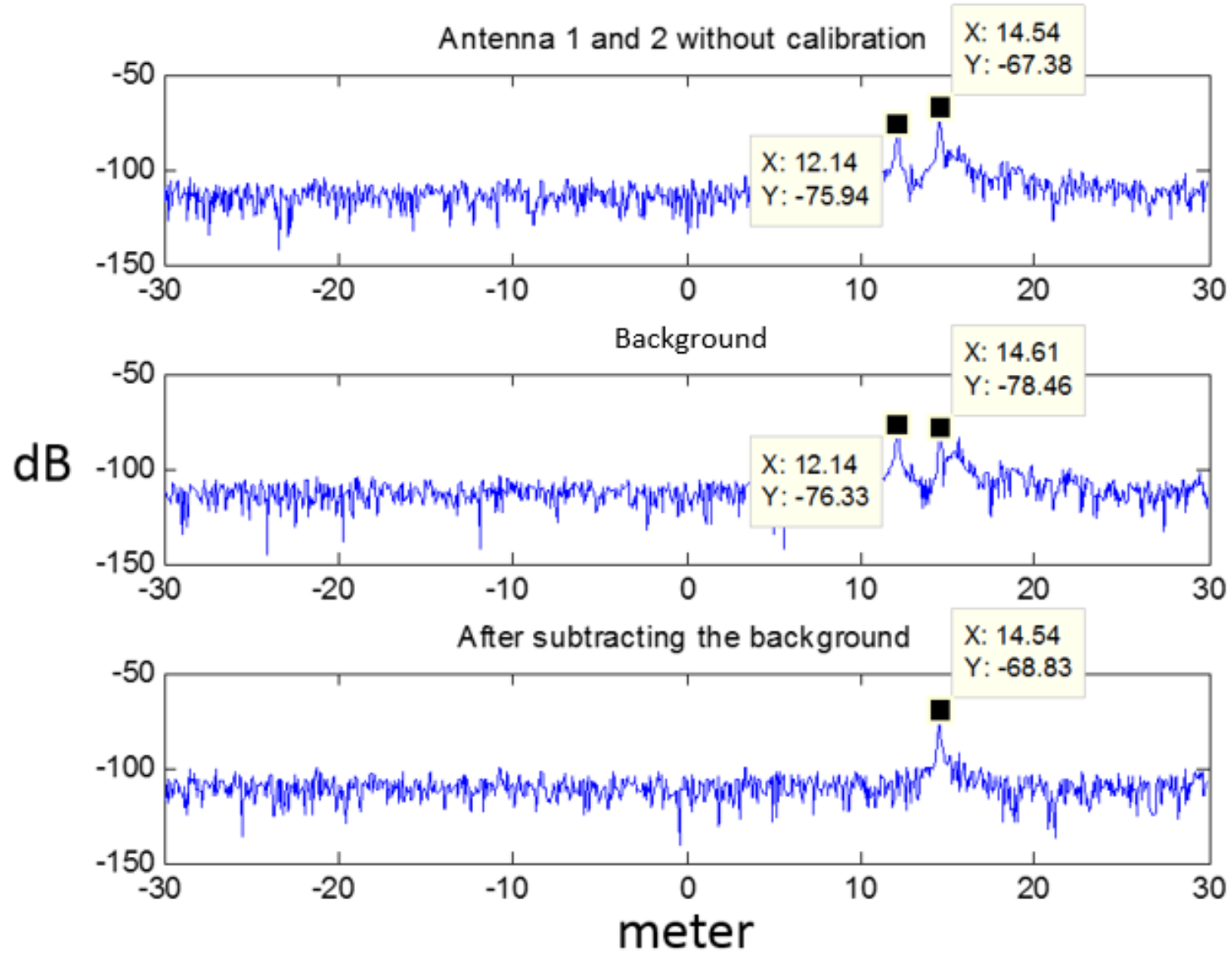

Figure 54: The scattering field of S-parameter S12 before calibration 


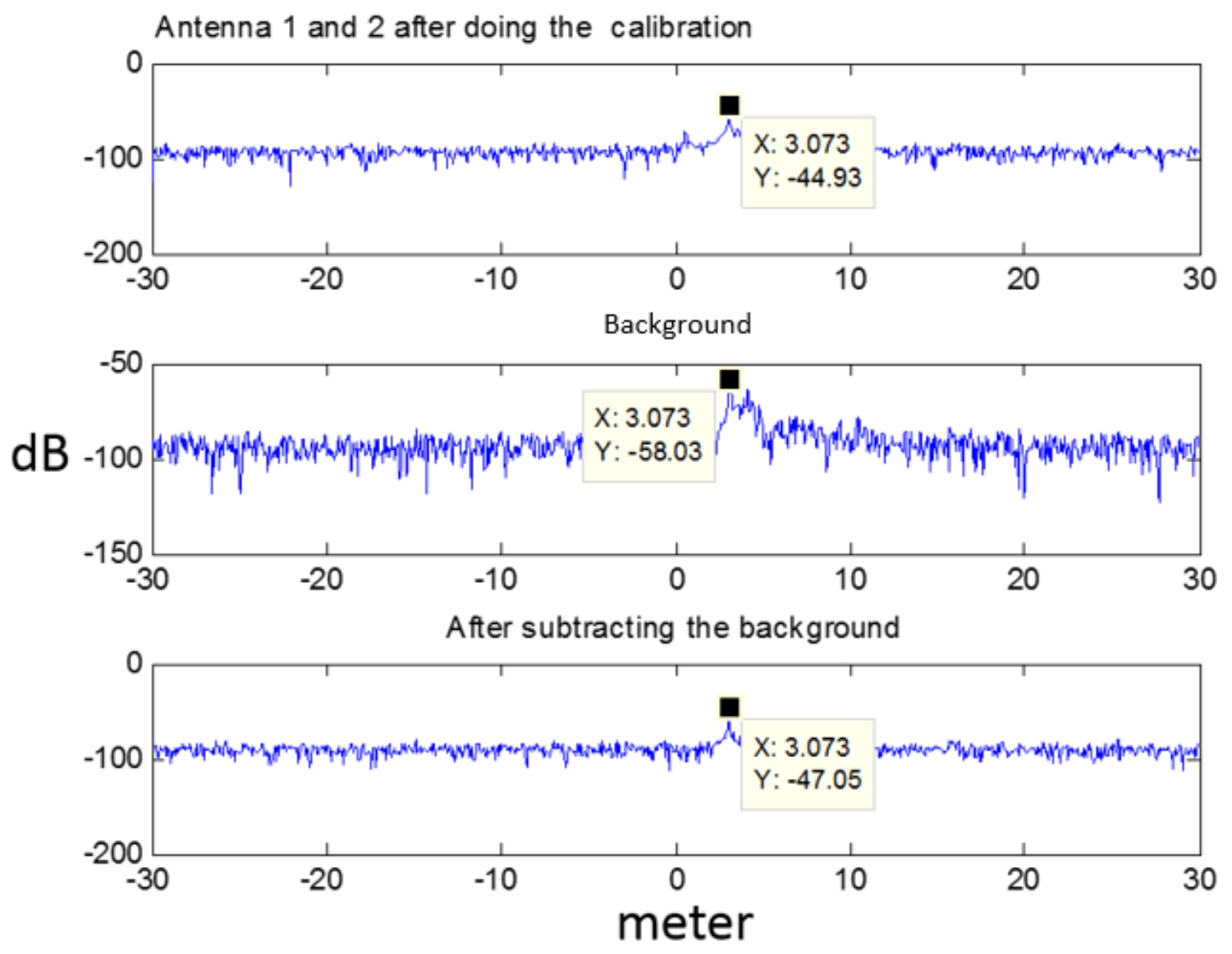

Figure 55: The scattering field of the S-parameter S12 after calibration.

\subsection{Three Cylinders Measurement}

Three cylinders were placed in diagonal line. The middle cylinder was placed at the center while other two cylinders were placed $33 \mathrm{~cm}$ away from the middle cylinder in opposite directions. The location of the middle cylinder determines the accuracy of the measurement. The electric scattering field was measured at 201 points for the X-band between $8 \mathrm{GHz}$ to $12 \mathrm{GHz}$. The middle cylinder was kept at $1.499 \mathrm{~m}$ for S51 and $1.537 \mathrm{~m}$ for S58 as shown in Figure 57 and Figure 58, respectively. The other cylinders have different locations due to the transmitting and receiving antennas. For example in Figure 57, the half traveling distance for the first cylinder was measured to be $1.349 \mathrm{~m}$, for the third cylinder, 
the half traveling distance was $1.762 \mathrm{~m}$. The traveling distance for the first cylinder changed due to the position of the antenna that was used in the measurement. Antennas 5 and 8 were used to measure the electric field response of the scene. The half traveling distance for the first cylinder was $1.237 \mathrm{~m}$ as shown in Figure 58 and $1.836 \mathrm{~m}$ for third cylinder.

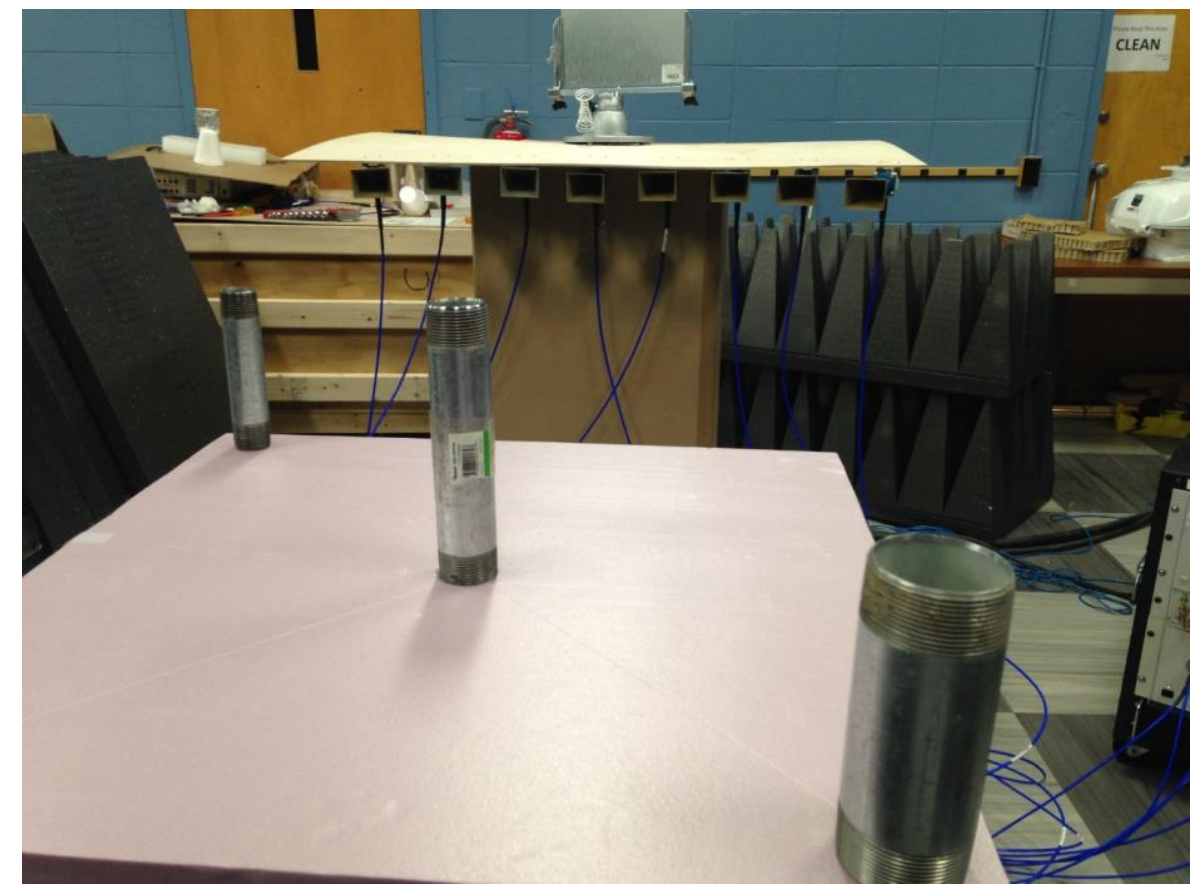

Figure 56: Three cylinders placed in diagonal form for measurement. 


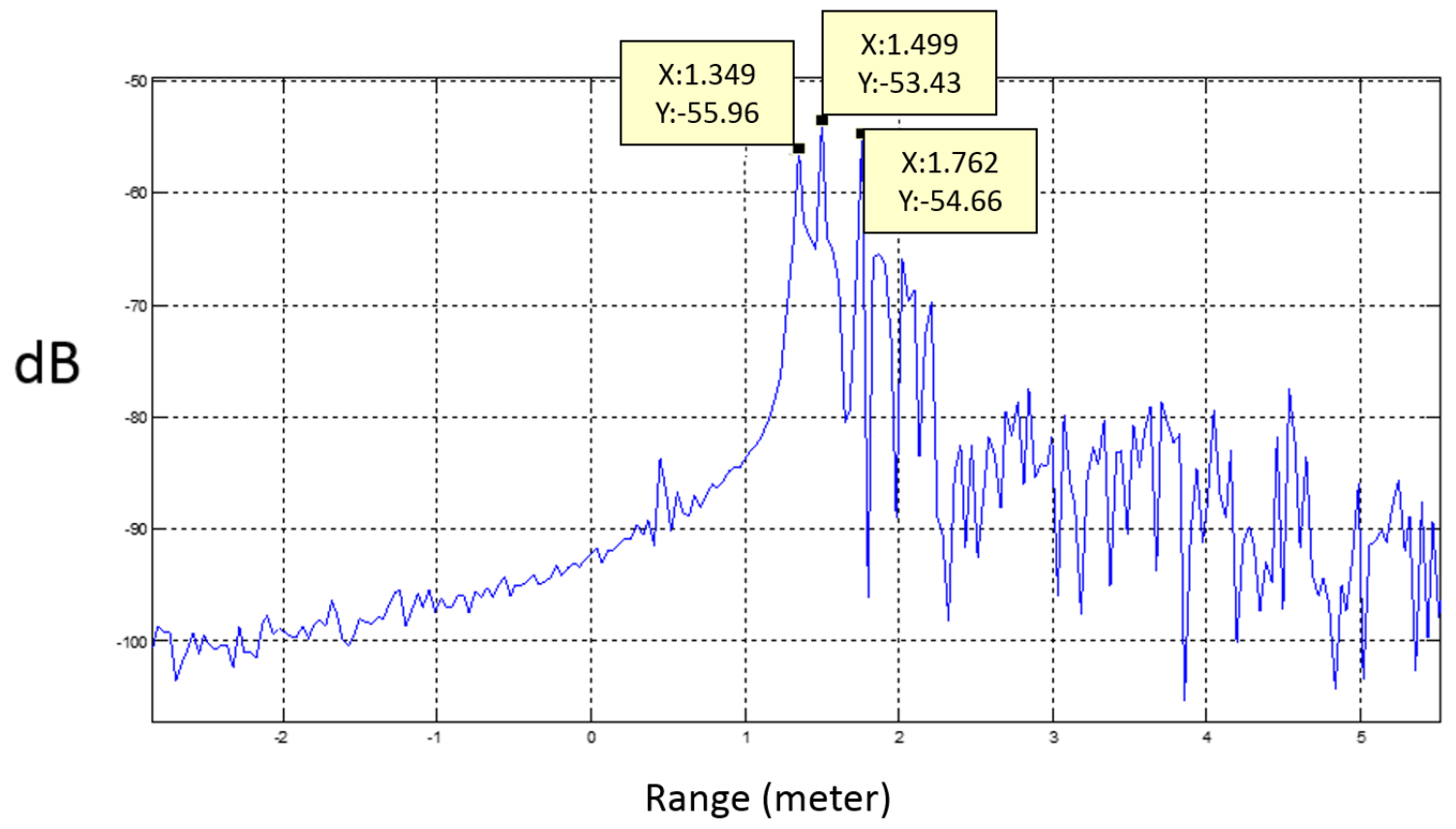

Figure 57: S15 the scattered field for the first antenna and fifth antenna

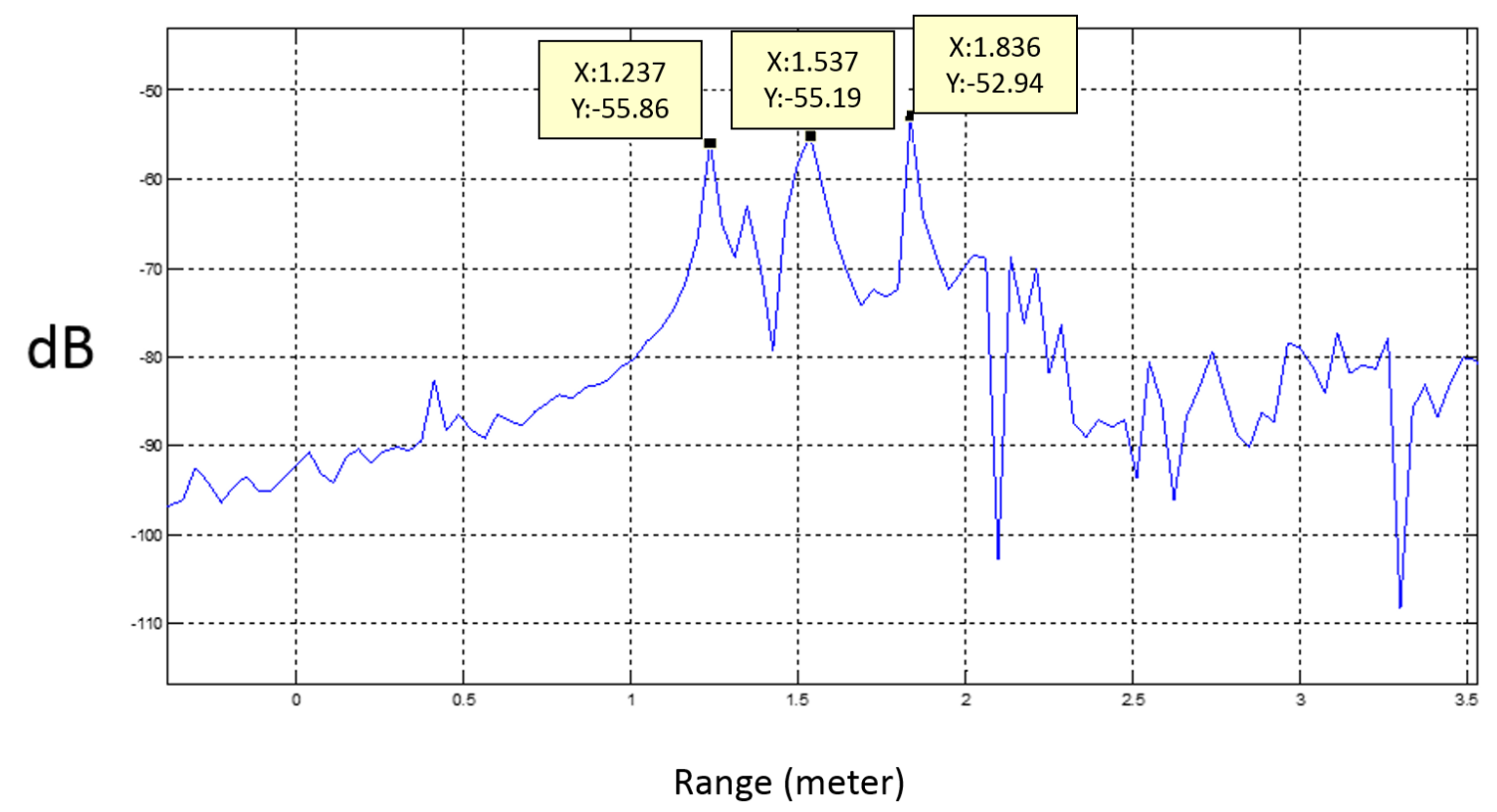

Figure 58: S85 the scattered field for the eighth antenna and fifth antenna

\subsection{Noise Effects}

Radar tomographic imaging systems suffer from many different types of noise. The effects of the noise cause an increase in the distortion of the image in high frequency. In 
this section, the appearance of natural noise in Mumma radar imaging system is discussed. However, noise occurrences due to errors in the mathematical model, neglecting some image processing parameters, or resulting from the inversion process is not presented.

The first source of noise is the noise figure; the noise figure is caused by the components in the Radio frequency system. The second noise is thermal noise, also known as the Johnson-Nyquist noise. Thermal noise is generated by thermal agitation in the microwave circuits. Thermal noise is inherent to any electrical circuit operating above absolute. Both noise figure and thermal noise were measured show in Figure 61 and PDF function was created for this type of measurement (Figure 60). Lowering the temperature of the electrical components will lower the thermal noise. This noise can be modeled as a simple resistor producing white Gaussian noise. The solution of these sources of noise is to use an amplifier and lower the anechoic chamber temperature. Mumma Radar Laboratory employs a private air conditioning system to set the chamber temperature at a desired sufficiently low temperature to minimize thermal noise within the RF frequency components. 


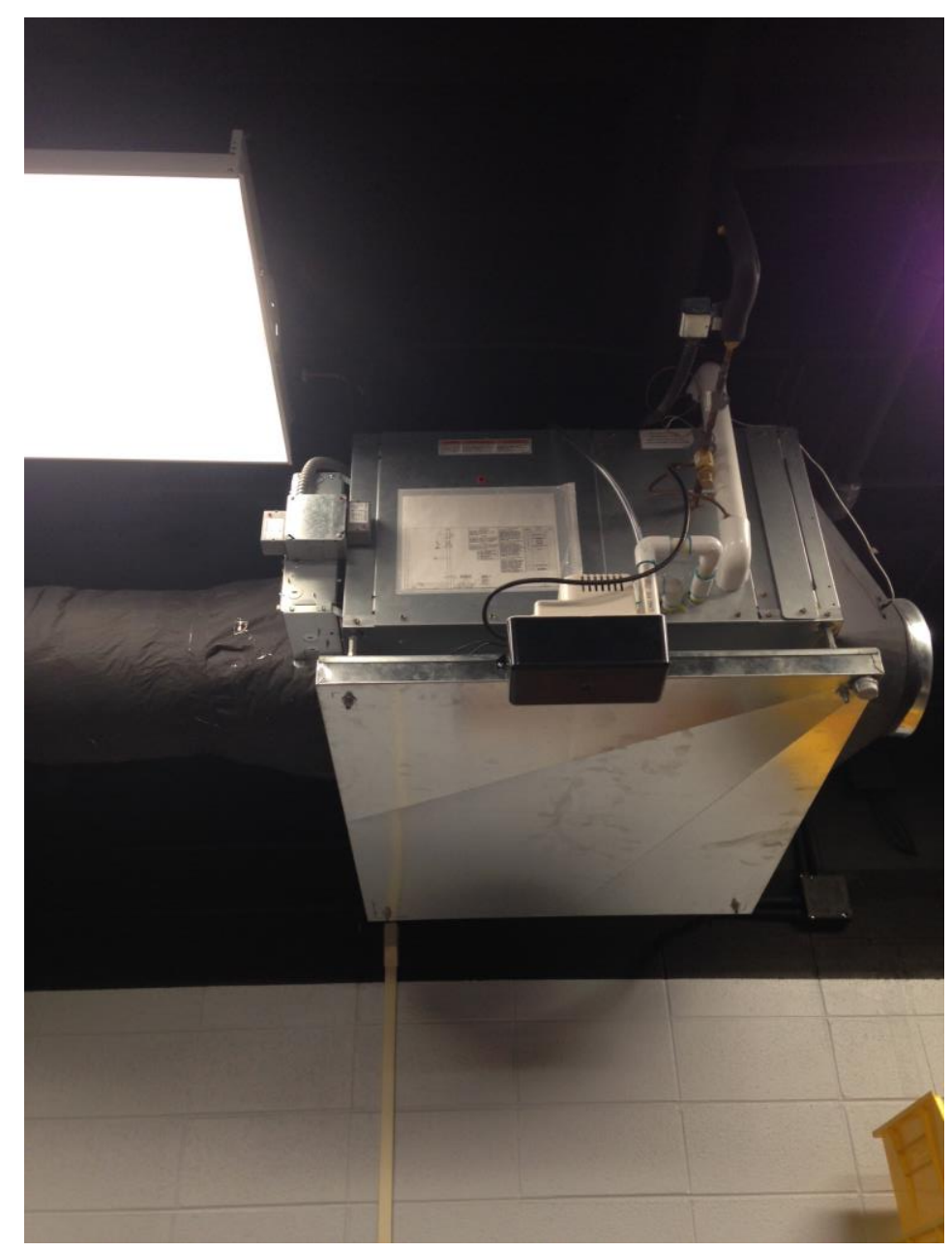

Figure 59: Air conditioning unit installed at the anechoic chamber.

Third source is mutual coupling caused by the interaction between the antennas in the Radar system and other antenna array systems. The mutual couple can be degraded by two options. First, background subtracting where we can measure the electric scattering field when target presents and target absent then subtracting them from each other. For real application, reversal time is used to have the same effects to unwanted signal or mutual coupling. The time reversal techniques transmit the received signal with opposite sign to subtract and attenuate undesired signal. Increasing the number of transmitters and receivers can sufficiently reduce the noise in the measurements. The trajectory of the scan will improve the noise immunity performance such scanning 360 degrees around the target. 
Increasing the sample rate of the points taking over the bandwidth is other way to reduce the noise, also will increase unambiguity of range and detection in Radar imaging system too. All these parameters can sufficiently lower SNR of the system and that will decrease the resolution as we mention the relationship between the SNR to the resolution previous chapter.

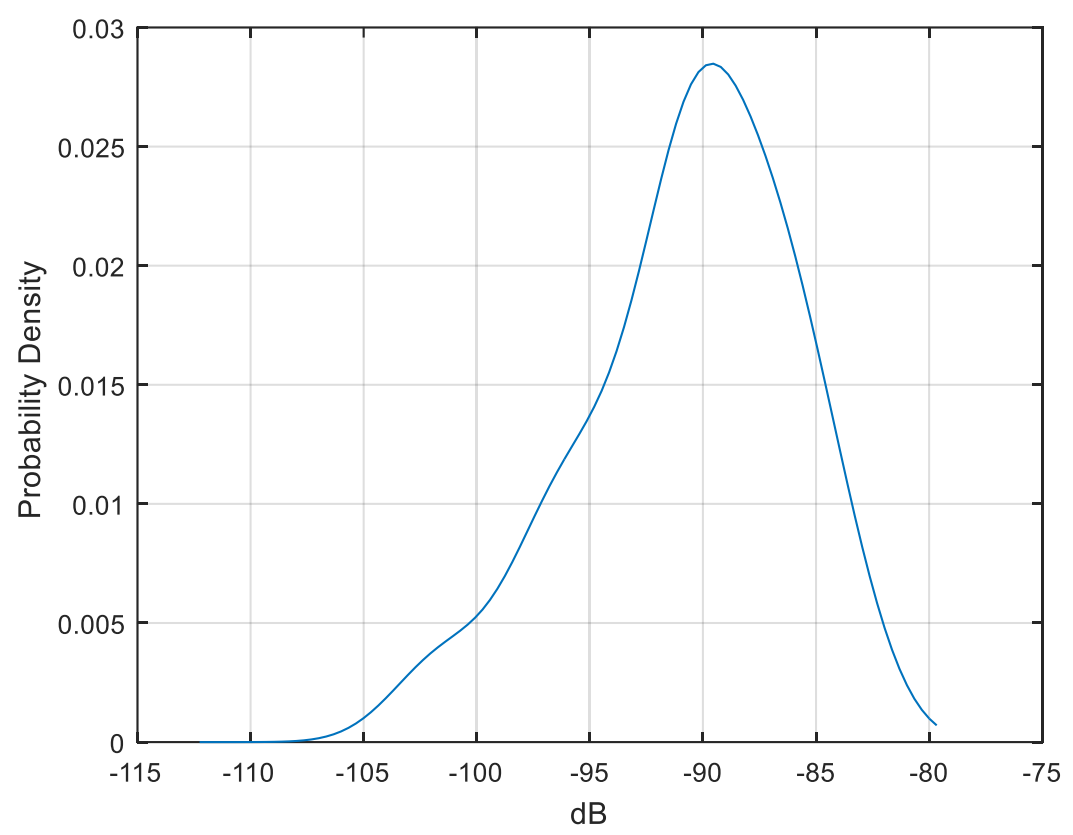

Figure 60: The probability density function of the white Gaussian noise at Mumma lab. 


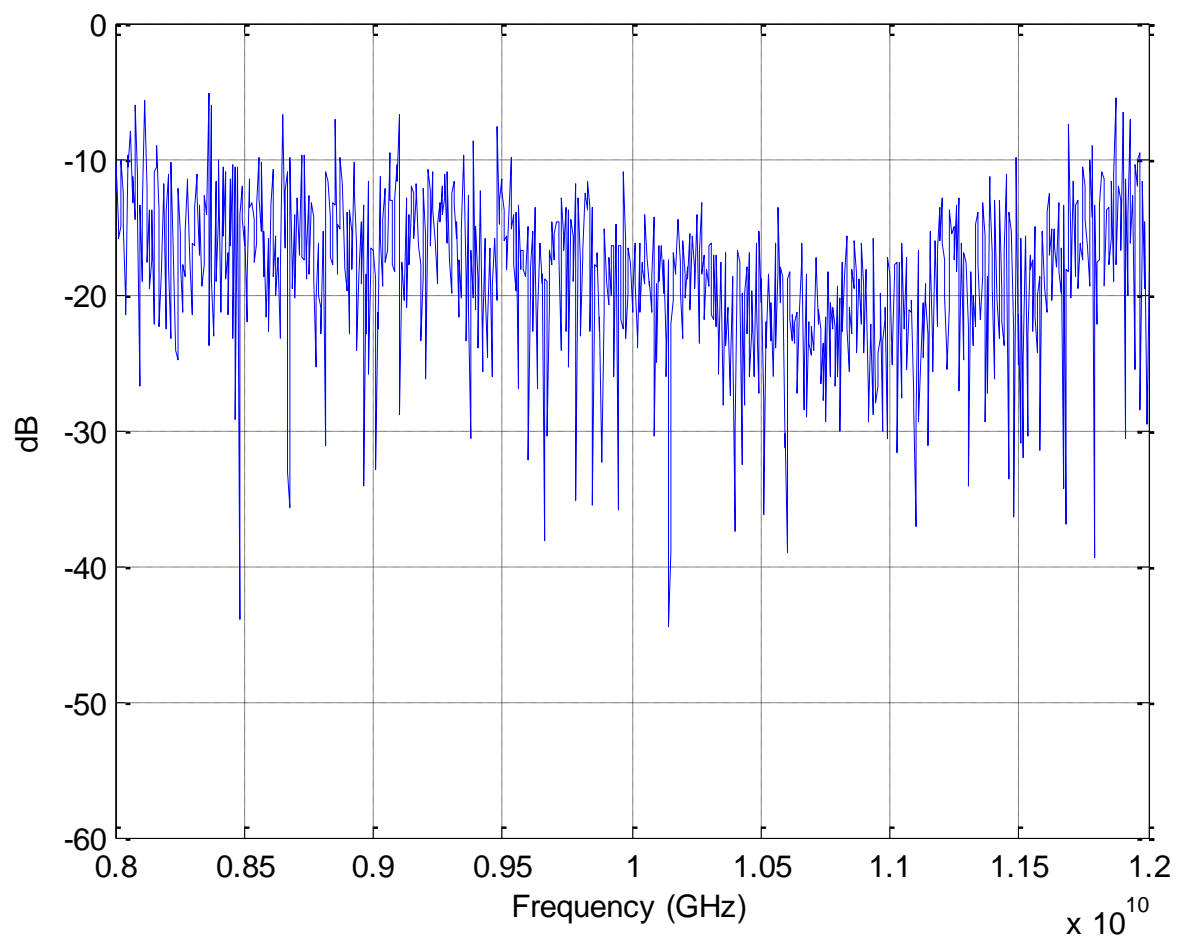

Figure 61: White Gaussian noise at Mumma lab in frequency domain. 


\section{CHAPTER 8}

\section{CONCLUSION AND FUTURE WORK}

\subsection{Conclusion}

An algorithm to suppress the sidelobes due to dominant scatterers in tomographic radar

images was proposed, examined, and studied. Accordingly, each dominant scatterer is replaced by an equivalent dipole source, and a new image is formed. The equivalents dipole is processed as extra transmitter in the measurement domain which predict the multipath effects, the multiple scattering between targets. The output no longer has dominant scatterers or their associated sidelobes. The results were positive showing an improved image with greater resolution of small objects and weak targets within the scene.

\subsection{Future Work}

The algorithm will be adopted for modified coherent marine radars used in Mumma Radar lab. The marine radar modified to work coherently and brought for many applications. One of the application will be imaging the free space for an airborne target using two coherent marine radars. Designing a detector to test each single pixel to detect noise over target for stop the algorithm if target absent. The algorithm already proposed to 
prescreen nonmetallic anti-personnel landmines by using more than one unmanned aerial vehicles. The nonmetallic anti-personnel landmines have weak scattering needs to be extract and detected. 


\section{REFERENCES}

[1] A. Kak and M. Slaney, "Principles of Computerized Tomographic Imaging," in Engineering, 1988, p. 344.

[2] W. R. Hendee and E. R. Ritenour, Medical Imaging Physics. 2002.

[3] L. Lo Monte, D. Erricolo, F. Soldovieri, and M. C. Wicks, "Radio frequency tomography for tunnel detection,” IEEE Trans. Geosci. Remote Sens., vol. 48, no. 3 PART 1, pp. 1128-1137, 2010.

[4] P. N. T. Wells, “Ultrasound imaging," Phys. Med. Biol., vol. 51, pp. R83-98, 2006.

[5] J. E. Aldrich, "Basic physics of ultrasound imaging.," Crit. Care Med., vol. 35, no. 5 Suppl, pp. S131-S137, 2007.

[6] J. A. Jensen, S. I. Nikolov, K. L. Gammelmark, and M. H. Pedersen, "Synthetic aperture ultrasound imaging.," Ultrasonics, vol. 44 Suppl 1, pp. e5-e15, 2006.

[7] L. Lo Monte, F. Soldovieri, D. Erricolo, and M. C. (University of D. Wicks, "Imaging below irregular terrain using RF tomography," ... Remote Sensing, ..., vol. 50, no. 9, pp. 3364-3373, 2012.

[8] M. C. Wicks, "RF Tomography with Application to Ground Penetrating Radar," IEEE, pp. 2017-2022, 2007.

[9] D. Olivadese, E. Giusti, D. Petri, M. Martorella, A. Capria, and F. Berizzi, "Passive ISAR with DVB-T signals," IEEE Trans. Geosci. Remote Sens., vol. 51, no. 8, pp. 4508-4517, 2013.

[10] D. Pastina, M. Sedehi, and D. Cristallini, "Passive bistatic ISAR based on geostationary satellites for coastal surveillance," IEEE Natl. Radar Conf. - Proc., pp. 865-870, 2010.

[11] F. Colone, D. Pastina, P. Falcone, and P. Lombardo, "WiFi-based passive ISAR for highresolution cross-range profiling of moving targets," IEEE Trans. Geosci. Remote Sens., vol. 52, no. 6, pp. 3486-3501, 2014.

[12] W. . b Qiu, E. . Giusti, A. . c Bacci, M. . Martorella, F. . Berizzi, H. Z. . Zhao, and Q. . Fu, "Compressive sensing for passive ISAR with DVB-T signal," in Proceedings International Radar Symposium, 2013, vol. 1, pp. 113-118. 
[13] Y. Guzel, T. M. Tran, M. C. Wicks, and L. Lo Monte, "RF Tomography for Ground Penetrating Radar: Simulation and Experimentation," IEE Int. Radar Conf., pp. 1-5, 2015.

[14] K. Kulpa, J. Misiurewicz, P. Samczynski, M. Smolarczyk, and M. Mordzonek, "SAR IMAGE ENHANCEMENT BY DOMINANT SCATTERER REMOVAL,” pp. 3-7.

[15] K. Kulpa, "The CLEAN type algorithms for radar signal processing," 2008 Proc. Microwaves, Radar Remote Sens. Symp. MRRS 2008, pp. 152-157, 2008.

[16] K. Kulpa, J. Misiurewicz, P. Samczynski, and M. Smolarczyk, "Multilook technique for dominant scatterer removal in SAR images," Microwaves, Radar Remote Sens. Symp. 2008, pp. 232-235, 2008.

[17] A. Nassib, M. C. Wicks, and L. Lo Monte, “A Dyadic Target Model for Multistatic SAR / ISAR Imaging," pp. 3-6.

[18] M. Gustafsson, "Multi-static synthetic aperture radar," 2003.

[19] J. T. Smith and H. F. Morrison, "Estimating equivalent dipole polarizabilities for the inductive response of isolated conductive bodies," IEEE Trans. Geosci. Remote Sens., vol. 42, no. 6, pp. 1208-1214, 2004.

[20] P. Meincke, "Linear GPR inversion for lossy soil and a planar air-soil interface," IEEE Trans. Geosci. Remote Sens., vol. 39, no. 12, pp. 2713-2721, 2001.

[21] R. Snieder, "The role of the Born approximation in nonlinear inversion," Inverse Probl., vol. 6, no. 2, pp. 247-266, 1999.

[22] R. Persico, R. Bernini, and F. Soldovieri, "The Role of the Measurement Configuration in Inverse Scattering From Buried Objects Under the Born Approximation," IEEE Trans. Antennas Propag., vol. 53, no. 6, pp. 1875-1887, 2005.

[23] T. J. Cui and W. C. Chew, "Diffraction tomographic algorithm for the detection of threedimensional objects buried in a lossy half-space," IEEE Trans. Antennas Propag., vol. 50, no. 1, pp. 42-49, 2002.

[24] J. DeLaurentis, "Multipath synthetic aperture radar imaging," IET Radar, Sonar Navig., vol. 5, no. 5, pp. 561-572, 2011.

[25] Y. Jin and J. M. F. Moura, "TR-SAR: Time Reversal Target Focusing in Spotlight SAR," in Conf. on Acoustics, Speech, and Signal Processing, 2007, vol. 2, pp. II-957-II-960.

[26] Y. Jin, J. M. F. J. M. F. Moura, N. O’Donoughue, M. T. Mulford, and A. A. Samuel, "Time Reversal Synthetic Aperture Radar Imaging In Multipath," in Proc. of the Asilomar Conference on Signals, Systems, and Computer, 2007, pp. 1812-1816.

[27] D. A. Garren, "Process for mapping multiple-bounce ghosting artifacts from radar imaging data," No. U.S. 6,646,593 B1. 
[28] R. R. Garren, D.A., Greeno, "Method for developing and using an image reconstruction algorithm for multipath scattering."

[29] D. Rosenberg, L., Gray, “Anti-jamming techniques for multichannel SAR imaging," IEE Proc. Radar Sonar Navig., vol. 3, pp. 234-242, 2006.

[30] C. P. Andre, D.B., Hill, R.D., Moate, "Multipath simulation and removal from SAR imagery," Algorithms Synth. Aperture Radar Imag. XV, Proc. SPIE, vol. 69700M, no. 6970, pp. 1-10, 2008.

[31] F. Natterer, The Mathematics of Computerized Tomography, vol. 29, no. 1. 2002.

[32] W. C. Chew, Waves and Fields in Inhomogeneous Media. NewYork: IEEE Press, 1995.

[33] T. M. Habashy, R. W. Groom, and B. R. Spies, "Beyond the Born and Rytov approximations: A nonlinear approach to electromagnetic scattering," Journal of Geophysical Research, vol. 98, no. B2. p. 1759, 1993.

[34] M. Born and E. Wolf, Principles of optics. 1999.

[35] W. C. Chew and Y. M. Wang, "Reconstruction of two-dimensional permittivity distribution using the distorted Born iterative method," IEEE Trans. Med. Imaging, vol. 9, no. 2, pp. 218-225, 1990.

[36] M. Moghaddam, W. C. Chew, and M. Oristaglio, "Comparison of the born iterative method and tarantola's method for an electromagnetic time-domain inverse problem," Int. J. Imaging Syst. Technol., vol. 3, no. 4, pp. 318-333, 1991.

[37] A. M. Tehrani and E. Slob, "Iterative extended Born approximation based on CG-FFT integral equation method for low-frequency 3D modeling," 2007.

[38] T. J. Cui, W. C. Chew, X. X. Yin, and W. Hong, "Study of re solution and super resolution in electromagnetic imaging for half-space problems," IEEE Trans. Antennas Propag., vol. 52, no. 6, pp. 1398-1411, 2004.

[39] C. Tai, "Dyadic Green functions in electromagnetic theory," IEEE Press, 1994.

[40] A. Abubakar and T. Habashy, "A closed-form expression of the electromagnetic tensor Green's functions for a homogeneous TI-anisotropic medium," Geosci. Remote Sens. ..., vol. 3, no. 4, pp. 447-451, 2006.

[41] W. C. Chew, M. S. Tong, and B. Hu, Integral Equation Methods for Electromagnetic and Elastic Waves, vol. 3, no. 1. 2008.

[42] L. Lo Monte, M. C. Wicks, and B. Yazici, "RF tomography for building penetration," IEEE Natl. Radar Conf. - Proc., pp. 420-424, 2011. 
[43] L. L. Monte, F. Soldovieri, I. Akduman, and M. C. Wicks, "Imaging under irregular terrain using RF tomography and numerical green functions," Antennas Propag. Soc. Int. Symp. (APSURSI), 2010 IEEE, no. 1, pp. 1-4, 2010.

[44] Y. Guzel, M. C. Wicks, and L. Lo Monte, "A Fast Matched-Filtered Approach for GPR,” no. 2, pp. 3-6.

[45] P. M. Morse, "Methods of Theoretical Physics," Am. J. Phys., vol. 22, no. 6, p. 410, 1954.

[46] Y. M. Wang and W. C. Chew, "An iterative solution of the two-dimensional electromagnetic inverse scattering problem," Int. J. Imaging Syst. Technol., vol. 1, pp. 100-108, 1989.

[47] T. J. Cui, W. C. Chew, and W. Hong, "New approximate formulations for EM scattering by dielectric objects," IEEE Trans. Antennas Propag., vol. 52, no. 3, pp. 684-692, 2004.

[48] J. A. Högbom, "Aperture synthesis with a non-regular distribution of interferometer baselines,” Astron. Astrophys. Suppl., vol. 15, pp. 417-426, 1974.

[49] J. Tsao and B. D. Steinberg, "Reduction of Sidelobe and Speckle Artifacts in Microwave Imaging: the Clean Technique.," IEEE Trans. Antennas Propag., vol. 36, no. 4, pp. $543-$ $557,1988$.

[50] I.-S. Choii and H.-T. Kim, "Two-Dimensional Evolutionary Programming-Based CLEAN,” IEEE Trans. Aerosp. Electron. Syst., vol. 39, no. 1, pp. 373-382, 2003.

[51] A. Nassib, N. Hopkins, L. Lo Monte, and M. C. Wicks, "FEKO Based ISAR Analysis for 3D Object Reconstruction."

[52] M. Pastorino, Microwave Imaging. New Jersey: John Wiley \& Sons, Inc, 2012.

[53] V. Picco, T. Negishi, D. Erricolo, and L. Lo Monte, "Dyadic Contrast Function for RF Tomography : Preliminary Results," in Antenna Measurements \& Applications (CAMA), 2014, pp. 1-4.

[54] R. B. Colton, "Radar in the United States Army History and Early Development at the Signal Corps Laboratories, Fort Monmouth, N.J.," Proc. IRE, vol. 33, no. 11, pp. 740753, 1945.

[55] R. Payne-scottt, "Visibility of small echoes on PPI displays," Proceeding IRE, vol. 36, pp. 180-196, 1947.

[56] J. FREEDMAN, “Resolution in Radar Systems,” Proc. IRE, vol. 39, pp. 813-818, 1951.

[57] T. Wang, X. Wang, Y. Chang, J. Liu, and S. Xiao, "Estimation of precession parameters and generation of ISAR images of ballistic missile targets," IEEE Trans. Aerosp. Electron. Syst., vol. 46, no. 4, pp. 1983-1995, 2010. 
[58] W. W. Camp, J. T. Mayhan, and R. M. O. Donnell, "Wideband Radar for Ballistic Missile Defense and Range-Doppler Imaging of Satellites," Lincoln Lab. J., vol. 12, no. 2, pp. 267-280, 2000.

[59] P. van Dorp, R. Ebeling, and A. G. Huizing, "High resolution radar imaging using coherent multiband processing techniques," Radar Conference, 2010 IEEE. pp. 981-986, 2010.

[60] Q. Yang, R. Min, Z. Cao, and Y. Pi, "Super-resolution SAR tomography focusing by lp Norm regularization-the FOCUSS algorithm," in 2012 IEEE Globecom Workshops, GC Wkshps 2012, 2012, pp. 1384-1388.

[61] J. W. Strutt and B. Rayleigh, "Wave theory of Light," Sci. Pap. by John William Strutt, Baron Rayleigh, Cambridge U. Press. Cambridge, Orig. Publ. Encycl. Br. XXIV, 1888, vol. III, 1887-, pp. 47-189, 1902.

[62] J. H. Dunn and D. D. Howard, "RADAR Target Amplitude, Angle, and Doppler Scintillation from Analysis of the Echo Signal Propagating in Space," IEEE Trans. Microw. Theory Tech., vol. 16, no. 9, pp. 715-728, 1968.

[63] L. Lo Monte, R. Vela, J. T. Parker, and C. J. Baker, "A Calibration Procedure for GroundBased RF Tomography," vol. 0, pp. 635-640, 2012. 